\title{
Conformal Field Algebras with Quantum Symmetry from the Theory of Superselection Sectors
}

\author{
Gerhard Mack $^{1}$ and Volker Schomerus ${ }^{2}$ \\ 1 Mathematics Department, Harvard University, Cambridge, MA02138, USA* \\ 2 II. Institut für Theoretische Physik, Universität Hamburg, W-2000 Hamburg 50, \\ Federal Republic of Germany
}

Received December 22, 1989; in revised form May 16, 1990

\begin{abstract}
According to the theory of superselection sectors of Doplicher, Haag, and Roberts, field operators which make transitions between different superselection sectors - i.e. different irreducible representations of the observable algebra are to be constructed by adjoining localized endomorphisms to the algebra of local observables. We find the relevant endomorphisms of the chiral algebra of observables in the minimal conformal model with central charge $c=\frac{1}{2}$ (Ising model). We show by explicit and elementary construction how they determine a representation of the braid group $B_{\infty}$ which is associated with a Temperley-LiebJones algebra. We recover fusion rules, and compute the quantum dimensions of the superselection sectors. We exhibit a field algebra which is quantum group covariant and acts in the Hilbert space of physical states. It obeys local braid relations in an appropriate weak sense.
\end{abstract}

\section{Table of Contents}

1. Introduction and Summary of Results . . . . . . . . . . . . . . . . . 140

2. The Observable Algebra and Its Positive Energy Representations . . . . . . . . 154

2.1. The Virasoro Algebra with $c=\frac{1}{2}$ and Associated Net of Local Observables . . . 154

2.2. Majorana Algebras on the Circle . . . . . . . . . . . . . . . . . . 157

2.3. The Lie Algebra of Local Observables . . . . . . . . . . . . . . . . . 159

2.4. Decomposition of Majorana Representations into Representations of Observable Algebras . . . . . . . . . . . . . . . . . . . . . . . . . . . 163

3. Endomorphisms which Intertwine Positive Energy Representations . . . . . . . 164

3.1. Endomorphisms of the Universal Majorana Algebra which Restrict to Endomorphisms of the Lie Algebra of Observables Lie $\overline{\mathscr{A}}$. . . . . . . . . . . . . 164

3.2. Action of Endomorphisms on Positive Energy Representations . . . . . . . . 165

3.3. Interpretation as Lifts of Endomorphisms of an Orthogonal Group. Index . . . 167

3.4. Localized Endomorphisms . . . . . . . . . . . . . . . . . . . . 170

* On sabbatical leave from II. Institut für Theoretische Physik, Universität Hamburg 
4. Fusion Rules and Intertwining Operators between Representations . . . . . . . 172

4.1. Fusion Rules . . . . . . . . . . . . . . . . . . . . . . . . . 172

4.2. Intertwiners in the Algebra . . . . . . . . . . . . . . . . . . . . 174

4.3. Intertwining Operators between Superselection Sectors . . . . . . . . . . 177

4.4. Operator Product Expansions . . . . . . . . . . . . . . . . . . . . 178

5. Braid Group . . . . . . . . . . . . . . . . . . . . . . . . . . . 180

5.1. Jones Algebra and Braid Group Representation Determined by the Endomorphism

$\varrho_{1 / 2}$. . . . . . . . . . . . . . . . . . . . . . . . . . . . . 180

5.2. Standard Braid Matrices . . . . . . . . . . . . . . . . . . . . . . 184

6. Fields with Quantum Symmetry $U_{q}(s l(2)), q=e^{-i \pi / 2}$. . . . . . . . . . . . . . . . 186

6.1. The Quantum Group $U_{q}(s l(2))$ with $q=-i-a$ a Brief Review. . . . . . . . . . 186

6.2. Field Transformation Law . . . . . . . . . . . . . . . . . . . . . . . . . . . 187

6.3. Quantum Group Covariant Field Algebras . . . . . . . . . . . . . . . . . . . . 189

6.4. Operator Product Expansions . . . . . . . . . . . . . . . . . . . . 193

A. Appendix. Local Braid Relations . . . . . . . . . . . . . . . . . . . 193

B. Appendix. Table 1. . . . . . . . . . . . . . . . . . . . . . . . . 194

\section{Introduction and Summary of Results}

According to the laws of local relativistic quantum mechanics, observables are selfadjoint operators acting in a Hilbert space $\mathscr{H}$ of physical states. The Hilbert space of physical states $\mathscr{H}$ may decompose into orthogonal subspaces $\mathscr{H}_{J}$, called superselection sectors, such that observables $A$ do not make transitions between different subspaces $\mathscr{H}_{J}[1]$. Different sectors $\mathscr{H}_{J}$ carry inequivalent irreducible positive energy representations of the algebra $\mathscr{A}$ of observables $A$, possibly with some multiplicity. Among the sectors is the vacuum sector $\mathscr{H}_{0}$. It contains the vacuum $|0\rangle$ and carries the vacuum representation $\pi_{0}$ of $\mathscr{A}$, with multiplicity 1 .

Typically, states in different sectors transform according to inequivalent unitary representations of a gauge group $G$ of first kind. By definition, a gauge group is a symmetry group of the theory which acts trivially on observables. Examples of sectors and associated gauge groups are

1. Fermi superselection sectors labelled by fermion number $F=0,1(\bmod 2)$

$$
|\psi\rangle \rightarrow(-1)^{F}|\psi\rangle
$$

gauge group $\mathbf{Z}_{2}$.

2. Baryon superselection sectors labelled by baryon number $B=0, \pm 1, \pm 2, \ldots$

$$
|\psi\rangle \rightarrow e^{i \alpha B}|\psi\rangle
$$

gauge group $U(1)$.

Nonabelian gauge groups are possible in principle. Suppose that isospin conservation were exact in the world, electromagnetism were absent, and particle detectors used gravitational interactions. Then the group $S U(2)$ of isospin rotations would be a nonabelian gauge group of the first kind. One could still detect that there are two kinds of nucleons - protons and neutrons - through the effect of their statistics (and by multiple scattering experiments). For instance, the high stability of the $\alpha$-particle comes about because 4 nucleons may be in the same orbital state. It is then natural to consider a Hilbert space with states that need not be isospin singlets and may contain arbitrary numbers of protons and neutrons. 
Superselection sectors are then labelled by isospin $I=0, \frac{1}{2}, 1, \ldots$, and occur with multiplicity $2 I+1$,

$$
\mathscr{H}=\bigoplus_{I} \bigoplus_{m=-I}^{I} \mathscr{H}_{I m} .
$$

Subspaces $\mathscr{H}_{I m}$ with the same isospin $I$ but different $3 \mathrm{rd}$ component $m$ of isospin carry equivalent representations of the observable algebra.

When superselection sectors exist, it is of interest to construct field operators $\phi, \psi \ldots$ which make transitions between sectors, and which are relatively local to the observables (Fig. 1).

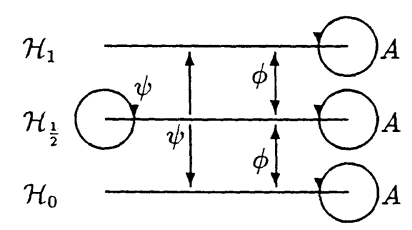

Fig. 1. Structure of the Hilbert space. Observables $A$ leave subspaces $\mathscr{H}_{J}$ invariant. The fields $\psi, \phi$ make transitions between subspaces $\mathscr{H}_{\boldsymbol{J}}$

In 2-dimensional conformal quantum field theory (CQFT) some algebras of chiral observables and their unitary irreducible positive energy representations are explicitly known, and the relative locality of other primary fields $\phi$ to observables like stress tensor $T$ or currents $j$ is embodied in local commutation relations. For instance,

$$
[T(w), \phi(z)]=\delta(z-\omega) \frac{d}{d z} \phi(z)+h \delta^{\prime}(z-w) \phi(z)
$$

for a chiral field $\phi$. Such fields depend on only one light cone variable, so that

$$
\text { relatively spacelike }=\text { disjoint }
$$

on the circle (see Sect. 2). In this paper we consider only chiral fields.

In the algebraic approach [2] one prefers to work with bounded operators like $e^{i T(f)}$ in place of operator valued distributions like $T(z)$, and similarly for fields $\phi$. Observables that are localized in bounded topological trivial domains $I$ of a Minkowskian space time manifold form a subalgebra $\mathscr{A}(I)$ of the observable algebra $\mathscr{A}$. Observables localized in relatively spacelike domains commute.

The basic ideas of the theory of superselection sectors of Doplicher, Haag, and Roberts are as follows [3]:

1. All the information about the theory is contained in the representation $\pi_{0}$ of the net of local algebras $\{\mathscr{A}(I)\}$ in the vacuum sector $\mathscr{H}_{0}$ and can be deciphered by looking at the localized endomorphisms $\varrho$ of $\mathscr{A}$.

2. The representations $\pi_{J}$ of $\mathscr{A}$ in other sectors are obtained from the vacuum representation $\pi_{0}$ by composition with suitable endomorphisms $\varrho_{J}$ of $\mathscr{A}$ :

$$
\pi_{J} \cong \pi_{0} \circ \varrho_{J}, \quad \text { with } \quad \pi_{0} \circ \varrho(A)=\pi_{0}(\varrho(A)) .
$$

3. The field algebra is obtained from the observable algebra $\mathscr{A}$ by adjoining outer endomorphisms of $\mathscr{A}$ to it. This means that an element $\phi$ of the field algebra should 
exist to every endomorphism $\varrho$ so that $\varrho$ becomes conjugation by $\phi$ or, more generally,

$$
A \phi=\phi \varrho(A) \text { for all } A \in \mathscr{A} \text {. }
$$

4. The endomorphisms $\varrho$ and their intertwiners determine the gauge group.

The observable algebra consists of complex linear combinations of selfadjoint observables. Therefore it admits a nontrivial *-operation (taking adjoints). By definition, an endomorphism is a linear map $\varrho: \mathscr{A} \rightarrow \mathscr{A}$ with the properties

$$
\begin{gathered}
\varrho(A B)=\varrho(A) \varrho(B), \\
\varrho\left(A^{*}\right)=\varrho(A)^{*}, \\
\varrho(\mathbf{1})=\mathbf{1} .
\end{gathered}
$$

It is called an automorphism if it has an inverse.

An intertwiner $T$ between endomorphisms $\varrho_{1}$ and $\varrho_{2}$ is an element $T \in \mathscr{A}$ of the observable algebra with the property

$$
T \varrho_{1}(A)=\varrho_{2}(A) T \text { for all } A \in \mathscr{A} .
$$

The linear space of all such intertwiners is denoted $\operatorname{Hom}\left(\varrho_{1}, \varrho_{2}\right)$.

It is easy to see that $\pi=\pi_{0} \circ \varrho$ is a unitary representation of $\mathscr{A}$ if $\pi_{0}$ is. That is

$$
\pi(A)=\pi_{0}(\varrho(A))
$$

has the defining properties of a unitary representation: $\pi\left(A_{1} A_{2}\right)=\pi\left(A_{1}\right) \pi\left(A_{2}\right)$ and $\pi\left(A^{*}\right)=\pi(A)^{*}$. Moreover, if the energy operator $H$ is positive, i.e. $H=\sum_{i} A_{i} A_{i}^{*} \geqq 0$, then $\varrho(H)=\sum_{i} \varrho\left(A_{i}\right) \varrho\left(A_{i}\right)^{*} \geqq 0$. Thus, $\pi=\pi_{0} \circ \varrho$ will be a positive energy representation if $\pi_{0}$ is. In the context of conformal quantum field theory, $H=L_{0}$.

Let us explain how these constructions work at a simplified example. Substitute the matrix groups $\mathscr{A}=S L(2, \mathrm{C})$ or $S O(2 N)$ for the algebra of observables, with the natural *-operation. There exists an outer automorphism $\varrho_{1}$ [complex conjugation for $S L(2, \mathrm{C})]$ :

$$
A \rightarrow \varrho_{1}(A) .
$$

In $S O(2 N)$ or $S O_{+}(3,1)=S L(2, \mathbf{C}) / \mathbf{Z}_{2}$ (proper orthochronous Lorentz group), the automorphism is conjunction by reflection. We adjoin an element $\Gamma$ which implements this automorphism in the sense that

$$
A \Gamma=\Gamma \varrho_{1}(A) .
$$

This yields a new group with elements $\Gamma^{n} A(n \in \mathbf{Z}, A \in \mathscr{A})$, since products of any two such elements may be brought into the same form by commuting $A$ 's through $\Gamma$ 's, using Eq. (1.9).

Automorphisms (and endomorphisms) can be multiplied, and it follows that $A \Gamma^{2}=\Gamma^{2} \varrho_{1}^{2}(A)$. But the automorphism $\varrho_{1}^{2}$ is trivial, thus $A \Gamma^{2}=\Gamma^{2} A$. We invoke now a new principle which says that elements of the field algebra (here: the new group) which commute with all observables should be in the center of the observable algebra. This leaves two possibilities $\Gamma^{2}= \pm 1$, since the center of $S O(2 N)$ or $S L(2, \mathbf{C})$ has only two elements \pm 1 . With either choice, we obtain a group with elements $\Gamma^{n} A(n=0,1 ; A \in S U(2))$. This "field group" substitutes for the field algebra in quantum field theory. 
The relation $\varrho_{1}^{2}=i d$ can be expressed in a fancier way by saying that unit operator $\mathbf{1} \in \operatorname{Hom}\left(\varrho_{1}^{2}, i d\right)$, viz. $1 \varrho_{1}^{2}(A)=A \mathbf{1}$ for all $A$. Thus, relations in the field group beyond (1.9) are linked to the existence of an intertwiner.

Our baby example admits $\mathbf{Z}_{2}$ as a gauge group. Its elements act as automorphisms of the field group which leave the observables fixed.

$$
\Gamma \rightarrow z \Gamma, \quad A \rightarrow A, \quad z= \pm 1 \in \mathbf{Z}_{2} .
$$

Passing from $S L(2, \mathrm{C})$ to the Lorentz group $\mathscr{A}=S O_{+}(3,1)$, the field group becomes $O_{+}(3,1)$. There are many space reflections, parametrized by planes, and corresponding automorphisms $\varrho$. But we need only adjoin one new element $\Gamma$. It is associated with some representative automorphism $\varrho_{1}$. All other automorphisms differ only by inner automorphisms $\sigma_{U}$ from $\varrho_{1}$, with unitary $U=U^{*-1}$. Later on we will wish to admit partial isometries $U$, therefore we write

$$
\sigma_{U}(A)=1+U^{*}(A-1) U .
$$

This will be an auto- or endomorphism whenever $U U^{*}=1$. All the reflections $\varrho$ are implemented by "field operators"

$$
\phi=\Gamma U
$$

with $U \in \mathscr{A}, U U^{*}=1$, in the sense that

$$
\begin{gathered}
A \phi=\phi \varrho(A), \\
\varrho=\sigma_{U} \varrho_{1} .
\end{gathered}
$$

If $\pi_{0}$ is a unitary irreducible representation of a compact group like $S O(2 N)$, and $\varrho$ is an automorphism, then $\pi_{0} \circ \varrho$ is another such representation. It has the same dimension as $\pi_{0}$. There is therefore no chance of obtaining all representations from a single one in this way. But all three eight-dimensional representations of $S O(8)$ are related by outer automorphisms, for instance.

Positive energy representations in CQFT are all $\infty$-dimensional. It is known [3] that $\pi_{J} \cong \pi_{0} \circ \varrho_{J}$ for some (localized) endomorphism $\varrho_{J}$, if the restriction of $\pi_{J}$ to the net of subalgebras $\left\{\mathscr{A}(I), I \subset I_{1}\right\}$ with $I_{1}=$ spacelike complement of some bounded, topological trivial domain in space-time, is unitarily equivalent to $\pi_{0}$, and if $\pi_{0}(\mathscr{A})$ satisfies Haag duality. In CQFT, such a spacelike complement is a compact subset of Minkowski space (see Sect. 2). All representations of nets of von Neumann algebras on such domains are known to be unitarily equivalent [4]. Therefore we can expect to obtain all positive energy representations from the vacuum representation $\pi_{0}$, provided our local algebras $\pi_{0}(\mathscr{A}(I))$ are von Neumann algebras satisfying Haag duality [5, Theorem 1$]$. Haag duality requires $\pi_{0}(\mathscr{A}(I))^{\prime}$ $=\pi_{0}\left(\mathscr{A}\left(I^{\prime}\right)\right) ; I^{\prime}$ is the spacelike complement of $I$ and $\pi_{0}(\mathscr{A}(I))^{\prime}$ consists of all bounded operators on $\mathscr{H}_{0}$ which commute with all operators $\pi_{0}(A), A \in \mathscr{A}(I)$. Haag duality is a maximality condition on the observable algebras, see below.

In the quantum field theory context, field algebras will be obtained from observable algebras in the same way as the group $O_{+}(3,1)$ was obtained from the group $S O_{+}(3,1)$, by adjoining elements $\Gamma^{J}$. They implement representative endomorphisms $\varrho_{J}$, one for each sector $\mathscr{H}_{J}$. Fields $\phi$ are given by "bosonization formulae." The bosons here are the observables. Thus Eqs. (1.9) and (1.12) of our baby example remain valid,

$$
A \Gamma^{J}=\Gamma^{J} \varrho_{J}(A) \text { for all } A \in \mathscr{A} .
$$


Field operators are given by the bosonization formula

$$
\phi=\Gamma^{J} U, \quad U \in \mathscr{A}, \quad U U^{*}=\mathbf{1} .
$$

They obey

$$
A \phi=\phi \varrho(A) \text { with } \varrho=\sigma_{U} \varrho_{J}
$$

and $\sigma_{U}$ as in Eq. (1.10). The locality properties of fields relative to observables are given by the localization properties of the endomorphisms $\varrho$ which they determine via Eq. (1.16).

By definition, endomorphism $\varrho$ is localized in $I$ if

$$
\varrho(A)=A \text { for } A \in \mathscr{A}\left(I^{\prime}\right)
$$

whenever $I$ and $I^{\prime}$ are relatively spacelike (=disjoint on $S^{1}$ ). $\varrho$ is called localized if it is localized on some domain $I$. We define supp $\varrho$ to be the smallest interval on which $\varrho$ is localized.

Let $\mathscr{F}(I)$ be spanned by the semigroup of field operators of the form (1.15) which induce endomorphisms $\varrho$ that are localized in $I$. It follows from Eq. (1.15) and (1.17) that fields are relatively local to observables in the sense that

$$
[\phi, A]=0 \quad \text { if } \quad A \in \mathscr{A}(I), \quad \phi \in \mathscr{F}\left(I^{\prime}\right)
$$

whenever $I^{\prime}$ is relatively spacelike (disjoint) to $I$.

Given the quantum numbers $J$ of the field $\phi$ of Eq. (1.15), its localization properties are entirely determined by the observable factor $U$. One may construct fields at a point $\phi(z)$ by taking limits, as is familiar from vertex operators associated with loop groups [6]

$$
\phi^{J}(z)=\lim _{n \rightarrow \infty} \mathcal{N}_{\varrho_{n}} \Gamma^{J} U_{n} \text { with } \operatorname{supp} \varrho_{n} \rightarrow\{z\} .
$$

Herein $\mathscr{N}_{\varrho_{n}}$ are suitable chosen real normalisation factors, and $\varrho_{n}=\sigma_{U_{n}} \varrho_{J}$. By multiplying with projection operators $E_{K}$ on the sectors $\mathscr{H}_{K}$, one obtains intertwining operators between sectors

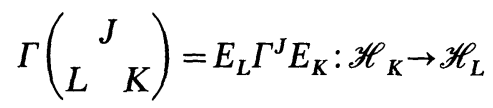

obeying

$$
\pi_{L}(A) \Gamma\left(\begin{array}{cc}
J \\
L & K
\end{array}\right)=\Gamma\left(\begin{array}{cc}
J \\
L & K
\end{array}\right) \pi_{K}\left(\varrho_{J}(A)\right)
$$

and chiral vertex operators

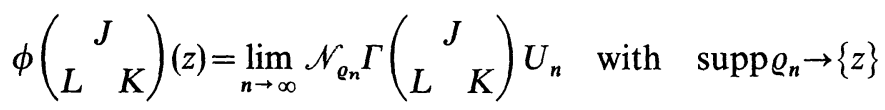

which satisfy the relations of an exchange algebra [7,8] [Eq. (1.59) below]. These are new kinds of vertex operators, not associated with lattices [10] unless $\varrho_{J}$ is an automorphism. Correlation functions

$$
\left\langle 0\left|\phi^{J_{1}}\left(z_{1}\right) \ldots \phi^{J_{n}}\left(z_{n}\right)\right| 0\right\rangle
$$


of fields (or vertex operators) may in principle be computed as follows. The pointlike limit (1.19) is only taken in the end. As a consequence of the product rule in the field algebra bounded field operators $\phi^{J}=\Gamma^{J} U$ satisfy operator product expansions (on the vacuum sector), they are found explicitly in Sects. 4.4, 6.4. Moreover

$$
\left\langle 0\left|\Gamma^{J} U\right| 0\right\rangle=\delta_{J, 0}\langle 0|U| 0\rangle .
$$

In this way, the correlation functions are expressed in terms of vacuum expectation values of observables. An illustration of this procedure for a model with automorphisms only is found in [5].

Application of the theory of superselection sectors in order to reconstruct a quantum field theory from the algebra of the stress energy tensor was first proposed by one of us in 1976 [42].

In this paper we consider the minimal 2-dimensional conformal field theory model with central charge $c=\frac{1}{2}$, which describes the scaling limit of the Ising model at the critical point. It has the Virasoro algebra $\mathrm{Vir}_{c=1 / 2}$ as a chiral Lie algebra of observables. This Lie algebra admits 3 inequivalent unitary irreducible positive energy representations $\pi_{J}$ in the Hilbert spaces $\mathscr{H}_{J}, J=0, \frac{1}{2}, 1$. They have lowest weights $\lambda_{J}=0, \frac{1}{16}, \frac{1}{2}$ for $J=0, \frac{1}{2}, 1$. The vacuum $|0\rangle \in \mathscr{H}_{0}$ is the lowest weight vector with $\lambda_{0}=0$. The local algebras of observables and the Virasoro algebra are generated by one light cone component of the stress energy tensor.

Interesting algebras of observables in conformal field theory are group algebras of $\infty$-dimensional Lie groups. In particular, the Virasoro algebra is the Lie algebra of a central extension $\operatorname{Diff}^{\sim}\left(\mathbf{S}_{1}\right)$ of (the universal covering of the diffeomorphism group of the circle. Therefore it is natural to work with Lie algebras as much as possible. There is nothing to guarantee that endomorphisms which we seek come from endomorphisms of Lie algebras, but in our model it turns out to be the case.

However, the associative algebras of local observables

$$
\mathscr{A}^{0}(I)=\operatorname{Span} \operatorname{Diff}^{\sim}(I) \subset \operatorname{Span} \operatorname{Diff}^{\sim}\left(\mathbf{S}^{1}\right)
$$

(diffeomorphisms of $\mathbf{S}^{1}$ that act trivially outside of $I$ ) do not have the property that $\pi_{0}(\mathscr{A}(I))$ are von Neumann algebras satisfying Haag duality.

Violation of the Haag duality condition $\pi_{0}(\mathscr{A}(I))^{\prime}=\pi_{0}\left(\mathscr{A}\left(I^{\prime}\right)\right)$ means that one can enlarge $\mathscr{A}^{0}(I)$ by adjoining further local operators that act on $\mathscr{H}_{0}$. It turns out that we need to consider enlarged Lie algebras to find suitable endomorphisms. Lie algebras of local observables, a global algebra Lie $\overline{\mathscr{A}}$ in which they are imbedded, and a complete set of endomorphisms $\varrho_{J}$ of Lie $\overline{\mathscr{A}}\left(J=0, \frac{1}{2}, 1\right)$ to reach all the sectors are found explicitly.

We summarize the result in

Theorem 1 (Endomorphisms). There exist local and global Lie algebras of observables, and injections

$$
i: \operatorname{Lie} \mathscr{A}(I) \rightarrow \operatorname{Lie} \overline{\mathscr{A}} \supset \text { Vir }
$$

such that all unitary irreducible positive energy representations $\pi_{J}$ of the Virasoro algebra Vir on a Hilbert space $\mathscr{H}_{J}$ extend to representations of Lie $\overline{\mathscr{A}}$ on the same Hilbert space $\mathscr{H}_{J}$, and

$$
\pi_{J} \cong \pi_{0} \circ \varrho_{J} \quad\left(J=0, \frac{1}{2}, 1 ; \varrho_{0} \equiv \mathrm{id}\right) .
$$


The endomorphisms $\varrho_{J}$ of Lie $\overline{\mathscr{A}}$ can be chosen so that they take the vacuum into lowest weight vectors, in particular

$$
\pi_{0}\left(\varrho_{J}\left(L_{0}\right)\right)|0\rangle=\lambda_{J}|0\rangle .
$$

$\varrho_{1 / 2}$ is not an automorphism.

The explicit description of Lie algebras and endomorphisms is found below and in Sects. 2 and 3. Lie $\overline{\mathscr{A}}$ is extension of the algebra of vector fields and functions on the circle (with generators $L_{n}=z^{-n} \frac{d}{d z}$ and $N_{n}=z^{-n}, n \in \mathbf{Z}$ ) by an orthogonal Lie algebra (with generators $J_{a b}, a, b \in \frac{1}{2} \mathbf{Z}, a-b \in \mathbf{Z}-\{0\}$ plus two central elements $\mathbf{1}$ and $Y)$.

The basic strategy in the proof of this part of the results is to construct everything in sight from Majorana fields, and to exhibit suitable homomorphisms of Majorana algebras, which induce endomorphisms of the global observable algebra. There are localized endomorphisms among them, but it is not convenient to choose them as representatives $\varrho_{J}$ since they could not take the vacuum into lowest weight vectors.

The Fourier modes $b_{a}=b_{-a}^{*}$ of the "universal" Majorana field

$$
\psi(z)=\sum_{a \in \frac{1}{2} \mathbf{Z}} b_{a} z^{-a-1 / 2}
$$

satisfy anticommutation relations

$$
\left\{b_{a}, b_{c}\right\}=\frac{1}{2} \delta_{a,-c}\left[1+(-1)^{2 a} Y\right] \text {. }
$$

Lie $\overline{\mathscr{A}}$ is spanned by bilinears $b_{a} b_{c}$ with $a-c \in \mathbf{Z}-\{0\}$ and central elements $1, Y . L_{n}$ and $N_{n}$ are infinite sums of such basis elements. The endomorphism $\varrho_{1 / 2}$ and the automorphism $\varrho_{1}$ act according to

$$
\begin{gathered}
\varrho_{1 / 2}\left(b_{a}\right)= \begin{cases}i b_{a+1 / 2} & a \geqq \frac{1}{2} \\
\frac{i}{\sqrt{2}}\left(b_{1 / 2}-b_{-1 / 2}\right) & a=0, \\
-i b_{a-1 / 2} & a \leqq-\frac{1}{2}\end{cases} \\
\varrho_{1}\left(b_{a}\right)= \begin{cases}-b_{a} & a \neq 0, \pm \frac{1}{2} \\
b_{-a} & a=0, \pm \frac{1}{2},\end{cases}
\end{gathered}
$$

The action of these endomorphisms on the unbounded operators $L_{n}, N_{n}$ is well defined.

Next we turn to the discussion of the field algebra with quantum symmetry. The example of isospin rotations in a world without electromagnetism, outlined at the beginning of this section, suggests a relation between gauge symmetry and statistics. This aspect cannot be illustrated in our baby example. Therefore it will be necessary to review some known facts before we state our result.

It has been known for a long time [3] that there exists an intrinsic definition of statistics of a superselection sector $\mathscr{H}_{J}$ if this sector can be reached by localized endomorphism $\varrho_{J}$, so that $\pi_{J} \cong \pi_{0} \circ \varrho_{J}$. Space time dimension $d \geqq 3$ was originally assumed, but it was recently pointed out by Fredenhagen, Rehren, and Schroer [8] that the definition applies in 2 dimensions as well, and leads to a representation of the braid group $B_{\infty}$ by operators in $\mathscr{A}(I) \subset \mathscr{A}$ for suitable $I \supset \operatorname{supp} \varrho_{J}$ which depends on $\varrho_{J}$. Since $\mathscr{A}\left(I_{1}\right) \subseteq \mathscr{A}\left(I_{2}\right)$ if $I_{1} \subseteq I_{2}$, suitable $I$ means large enough $I$. 
The analysis assumes that the localized endomorphism $\varrho=\varrho_{J}$ is transportable in the following sense. Given arbitrary $I_{i} C I$, unitaries $U_{i} \in \mathscr{A}(I)$ should exist such that $\varrho_{i}=\sigma_{U_{i}} \varrho$ is localized in $I_{i}$. In conformal field theory, transportability follows from covariance under diffeomorphisms if $\operatorname{Diff}^{\sim}(I) \cong \mathscr{A}(I)$ for intervals $I \subset \mathbf{S}^{1}$.

The generators $\sigma_{n}$ of the braid group $B_{\infty}$ have the form

$$
\sigma_{n}=\varrho^{n-1}\left(\varepsilon_{\varrho}\right) \quad n=1,2, \ldots
$$

Herein $\varepsilon_{Q}=\sigma_{1}$ is unitary and satisfies

$$
\left[\varepsilon_{\varrho}, A\right]=0 \text { for all } A \in \varrho^{2}(\mathscr{A}) \text {. }
$$

It is defined as follows $[3,8]$. Select disjoint intervals $I_{1}, I_{2} C I$ and unitaries $U_{i} \in \mathscr{A}(I)$ such that $\varrho_{i}=\sigma_{U_{i}} \varrho$ is localized in $I_{i}(i=1,2)$. Set

$$
\varepsilon_{\varrho}=\varrho\left(U_{1}\right) U_{2} U_{1}^{-1} \varrho\left(U_{2}^{-1}\right) \in \mathscr{A}(I) .
$$

It is known that this quantity is a homotopy invariant in the following sense. Given localization regions $I_{1}, I_{2}, \varepsilon_{e}$ does not depend on the choice of $U_{i}$. It is also the same for any pairs $\left(I_{1}, I_{2}\right)$ and $\left(I_{1}^{\prime}, I_{2}^{\prime}\right)$ that can be continuously deformed into each other, within $I$, maintaining disjointness. For intervals this means that $\varepsilon_{Q}$ depends only on whether $I_{1}$ is to the left or right of $I_{2}$.

We desire to work with representative endomorphisms $\varrho_{J}$ which are not localized, but are limits of localized endomorphisms $\varrho$, and with a global algebra $\mathscr{A}$ whose vacuum representation is not faithful. We abbreviate

$$
\varepsilon_{J} \equiv \varepsilon_{Q_{J}} \in \mathscr{A} .
$$

There remains some arbitrariness in expressions (1.31) for $\varepsilon_{J}$ if we weaken the requirement $U_{i} \in \mathscr{A}(I)$ to $U_{i} \in \mathscr{A}$, because we may multiply $U_{i}$ with different elements of the center of $\mathscr{A}$. The "Minkowski space" choice can be determined as follows. Select $\zeta \in S^{1}$ ("projection of the point at infinity of a Minkowski space"see Sect. 2). Consider localized endomorphisms $\varrho$ with supp $\varrho \subset I \not \zeta$ and define $\varepsilon_{\varrho}$ by Eq. (1.31) with $U_{i} \in \mathscr{A}(I)$. Inject $\varepsilon_{\varrho} \in \mathscr{A}(I) \rightarrow \mathscr{A}$. Then take the limit $\varrho \rightarrow \varrho_{J}$. The result may depend on $\zeta$. But it yields a representation of $B_{\infty}$ in any case, and $\varepsilon_{J}$ is invariant under deformations of disjoint pairs of intervals $\left(I_{1}, I_{2}\right)$ with $\zeta \notin I_{1} \cup I_{2}$.

In practise, the braid group representations are determined by seeking suitable $\varepsilon_{J}$ in the commutant of $\varrho_{J}^{2}(\mathscr{A})$, cp. Eq. (1.30).

In $d \geqq 3$-dimensional Minkowski space there is only one homotopy class of relatively spacelike bounded, topologically trivial domains, and the braid group representation degenerates into a permutation group representation (i.e. $\left.\sigma_{i}^{-1}=\sigma_{i}\right)^{1}$. Using this fact, Doplicher and Roberts [13] were able to describe a general construction of a field algebra $\mathscr{F}$, together with a compact gauge group $G$ which acts as a group of automorphisms of $\mathscr{F}$, such that the $G$-invariant elements of the field algebra $\mathscr{F}$ are precisely the observables $A \in \mathscr{A}$. Clearly a gauge group with this property cannot be trivial, if there exist superselection sectors $\mathscr{H}_{K} \neq \mathscr{H}_{0}$ that are generated by localized endomorphisms. Nonabelian gauge groups $G$ are obtained from endomorphisms which are not automorphisms, while automorphisms yield abelian gauge groups.

\footnotetext{
1 Braid group representations in 3 dimensions as were recently discussed by Fröhlich et al. and Fredenhagen $[11,12]$, come from endomorphisms which are not localized in bounded domains, but in unbounded cones in Minkowski space
} 
The physical Hilbert space $\mathscr{H}$ will be representation space for $\mathscr{F}$ and $G$. A nonabelian gauge group (or quantum group) $G$ with irreducible representations that are not 1 -dimensional requires that superselection sectors $\mathscr{H}_{J}$ occur with some multiplicity $d_{J}$,

$$
\mathscr{H}=\bigoplus_{J} \bigoplus_{m=1}^{d_{J}} \mathscr{H}_{J m}
$$

where $\mathscr{H}_{J m}$ carry the same representation $\pi_{J}$ of $\mathscr{A}$ for different $m$. The fields also come in multiplets $\phi_{m}^{J}, m=1, \ldots, d_{J}$, which transform covariantly under $G . d_{J}$ is determined by the permutation group representation associated with $\varrho_{J}$ in Doplicher and Roberts theory.

It is also asserted in the work of Doplicher and Roberts that the fields $\phi_{m}^{J}$ can be chosen to satisfy local commutation or anticommutation relations when they are localized in relatively spacelike domains. The nucleon field in the hypothetical world with exact isospin invariance is an example of a doublet of local Fermi fields which transform covariantly under gauge group $S U(2)$. Only commutation relations of fields $\phi_{m}^{J}$ with the same "charge" $J$ are intrinsically determined though, others can be changed by Klein transformations.

One expects that some sort of "quantum symmetry" will take the place of the gauge group $G$ in 2-dimensional quantum field theories with nontrivial braid group representations (1.29). But there exists no general theory yet which would identify this quantum symmetry.

In our model we find

$$
U_{q}(\operatorname{sl}(2)) \text { with } q=-i
$$

as a quantum symmetry. This quantum group algebra has generators $X=S_{ \pm}$and $q^{ \pm S_{z} / 2}$ and admits 3 "physical representations" $\tau^{J}$ of dimension $2 J+1$. They are labelled by quantum isospin $J=0, \frac{1}{2}, 1$. In Sect. 6.1 and 6.2 we will present a brief review of quantum group theory and an explanation of the notion of covariance of a quantum field theory under quantum groups. It appears natural if the quantum group algebra is thought of as a generalization of the enveloping algebra of a Lie algebra. In the statement of Theorem 2 below, the explicit form of comultiplication and counit in $U_{q}(s l(2))$ is used.

Basically, Theorem 2 is an assertion about properties of operators $\Gamma_{m}^{J}$. In this paper we concentrate on Lie algebras. The elements $A$ of the observable algebra $\mathscr{A}$ in Theorem 2 can be in the Lie algebra of observables Lie $\overline{\mathscr{A}}$, or in its universal enveloping algebra, or in other associative algebras $\mathscr{A}$ which are affiliated with Lie $\overline{\mathscr{A}}$ and to which the positive energy representations and endomorphisms $\varrho_{J}$ of Lie $\bar{A}$ can be extended. To obtain nonempty local field algebras $\mathscr{F}(I), \mathscr{A}$ needs to be big enough. In particular we want $\operatorname{Diff}^{\sim}(I) \subset \mathscr{A}$. It is fairly obvious what the natural associative algebra of observables should be. The group algebra of a (2-dimensional) central extension of the identity component of the restricted orthogonal group is a natural candidate for the global algebra. It is similar to the well known restricted unitary group [14]. However, we do not wish to enter into a mathematical discussion of this group and its endomorphisms in this paper. Therefore, results which require associative algebras larger than the universal enveloping algebra will be formulated as remarks.

At a heuristic level, we may think of the elements $U$ of the restricted orthogonal group as exponentials of quadratics in Majorana fields $\psi$, so that the bosonization 
formula takes the form

$$
\phi_{m}^{1 / 2}=\Gamma_{m}^{1 / 2} \exp i \int \psi(z) \mathscr{K}(z, w) \psi(w) d z d w,
$$

for $J=\frac{1}{2}$, with some kernel $\mathscr{K}$ which determines localization properties of $\phi_{m}^{1 / 2}$. The bosonization formula for $\phi_{m}^{1}$ merely reconstructs a triplet of Majorana field.

Theorem 2 (Field Algebra with Quantum Symmetry). Generators of a field algebra

$$
\phi_{m}^{J}=\Gamma_{m}^{J} U, \quad U \in \mathscr{A}, \quad U U^{*}=1 \quad\left(J=0, \frac{1}{2}, 1, m=-J, \ldots, J\right)
$$

and generators $X$ of the quantum group algebra $\mathscr{G}=U_{q}(s l(2))$ with $q=-i$ both act in a Hilbert space of physical states

$$
\mathscr{H}_{\text {phys }}=\underset{J=0, \frac{1}{2}, 1}{\oplus} \bigoplus_{m=J}^{J} \mathscr{H}_{J m}
$$

and obey the following covariance conditions.

1. Fields $\phi$ implement endomorphisms

$$
A \phi_{m}^{J}=\phi_{m}^{J} \varrho(A) \text { with } \varrho=\sigma_{U} \varrho_{J}
$$

or, equivalently, $A \Gamma_{m}^{J}=\Gamma_{m}^{J} \varrho_{J}(A)$.

2. The vacuum is quantum group invariant,

$$
S_{ \pm}|0\rangle=0, \quad q^{ \pm S_{z} / 2}|0\rangle=|0\rangle .
$$

3. Observables are quantum group invariant,

$$
[X, A]=0 \text { for } A \in \mathscr{A}, \quad X \in \mathscr{G} .
$$

4. The field operators are quantum group covariant in the sense that they obey generalized commutation relations with the quantum group generators,

$$
\begin{gathered}
q^{ \pm S_{z}} \phi^{J}=\phi^{J} \tau^{J}\left(q^{ \pm S_{z}}\right) q^{ \pm S_{z}}, \\
S_{ \pm} \phi^{J}=\phi^{J} \tau^{J}\left(q^{S_{z} / 2}\right) S_{ \pm}+\phi^{J} \tau^{J}\left(S_{ \pm}\right) q^{-S_{z} / 2} .
\end{gathered}
$$

5. Local braid relations (cp. Remark 3): The relation

$$
\Gamma_{k}^{J} \Gamma_{l}^{J} \varepsilon_{J}|\xi\rangle=\sum_{m, n} \Gamma_{m}^{J} \Gamma_{n}^{J}|\xi\rangle \mathscr{R}_{k l}^{m n}(J)
$$

holds true for vectors $|\xi\rangle$ in a subspace of $\mathscr{H}_{\mathrm{phys}}$ which includes $\mathscr{H}_{\mathrm{Kr}}$ if $J+K \leqq 1 . \mathscr{R}$ is the quantum group $\mathscr{R}$-matrix multiplied with a phase factor, for $J=\frac{1}{2}$.

We used matrix notation in $4 . \tau^{J}$ is the $2 J+1$ dimensional matrix representation of $\mathscr{G}$, and $\phi^{J}$ is the row vector with components $\phi_{m}^{J}$. Validity of 4 for general $\phi^{J}$ follows from its validity for $\phi_{m}^{J}=\Gamma_{m}^{J}$ by property $3 . \varepsilon_{J}$ defines the representation of the braid group as explained before.

The fields $\phi=\phi^{1 / 2}$ (chiral Ising field) and $\psi=\phi^{1}$ make transitions between sectors as specified in Fig. 1.

The significance of Relation 5 comes from its corollary as stated in the following:

Remark 3 (Local Braid Relations). Suppose that fields $\phi_{k}^{J}=\Gamma_{k}^{J} U$ and $\phi_{l}^{\prime J}=\Gamma_{l}^{J} U^{\prime}$ are localized on disjoint intervals on $\mathbf{S}^{1} \backslash\{\zeta\}$, and $U, U^{\prime}$ are limits of elements of local 
algebras $\mathscr{A}(I)$ with $I \not \zeta \zeta$. Then the fields obey local braid relations

$$
\phi_{k}^{J} \phi_{l}^{\prime J}|\xi\rangle=\sum_{m, n} \phi_{m}^{\prime J} \phi_{n}^{J} \mathscr{R}_{k l}^{m n}(J)|\xi\rangle
$$

on the subspace of states $|\xi\rangle$ where Relation 5 of Theorem 1 holds.

Local Braid relations of the form (1.42) were introduced by Fröhlich [15].

This corollary of identity 5 of Theorem 2 is established by a short and instructive calculation which uses the definition and homotopy invariance of $\varepsilon_{J} \equiv \varepsilon_{Q_{J}}$ (see Appendix A). $\zeta$ is the point on the circle which entered the definition of $\varepsilon_{Q_{J}}$.

We were unable to construct a field algebra which obeys local braid relations on all of $\mathscr{H}_{\text {phys }}$, with a matrix $\mathscr{R}$ that is independent of the sector to which the state $|\xi\rangle$ belongs. The reason is easy to explain.

The third component of quantum isospin will be called charge for short. Charge is conserved. Consider the chiral Ising field, $\phi^{J}, J=\frac{1}{2}$ and inspect the desired equations (1.41),(1.42) for states $|\xi\rangle \in \mathscr{H}_{K r}$ of isospin $K=1$ and charge $r=1$. Take it for granted that braid relations are nontrivial in the sense that matrix $\mathscr{R}\left(\frac{1}{2}\right)$ is nondiagonal. The canonical $\mathscr{R}$-matrix for $U_{q}(s l(2)), q=-i$ is nondiagonal for $J=\frac{1}{2}$. Then $\mathscr{R}_{-1 / 2,1 / 2}^{1 / 2} \neq 0$. Let $l=\frac{1}{2}$ and $k=-\frac{1}{2}$. Then the left-hand side of Eqs. (1.41), (1.42) is zero because there is no state of charge $\frac{3}{2}$ in $\mathscr{H}_{\text {phys }}$. But the righthand side receives one nonvanishing contribution, $m=\frac{1}{2}, n=-\frac{1}{2}$. This contribution cannot vanish for all $|\xi\rangle \in \mathscr{H}_{11}$ unless $\phi^{1 / 2}$ vanishes identically on one of the sectors, because the intertwining operators (1.20) satisfy completeness relations (Sect. 4.2).

It appears thus that local braid relations as an operator identity, with a nondiagonal numerical matrix $\mathscr{R}$, would require a space with unphysical states. It will be remembered that local (anti)commutation relations of charged fields in gauge theories like Quantum Electrodynamics also require an unphysical state space.

Theorem 2 is established in several steps which may be summarized as follows:

$\begin{aligned} & \rightarrow \begin{array}{l}\text { fusion rules } \rightarrow \text { intertwining operators } \Gamma\left(\begin{array}{cc}J \\ K & L\end{array}\right) \\ \text { endomorphisms }\end{array} \rightarrow \text { cov. field algebra } \\ & \longrightarrow \text { braid group representation } \rightarrow \text { local braid relations } .\end{aligned}$

In our baby example a preliminary field group was first constructed, with relations $A \Gamma=\Gamma \varrho_{1}(A)$. The existence of an intertwiner was then used to impose a further relation, $\left(\Gamma^{2}= \pm 1\right)$. In the conformal model we proceed in a slightly different way which is suggested by the work of Fredenhagen, Rehren, and Schroer [8]. Instead of displaying further relations, the field algebra is constructed as an algebra of operators in a Hilbert space, using the intertwiners as building blocks. Their group theoretical meaning becomes clear from the following consideration.

In the approach based on endomorphisms, all representations of interest are realized in one standard Hilbert space $\mathscr{H}_{0}$. In particular the irreducible representations $\left(\pi_{K}, \mathscr{H}_{K}\right) \cong\left(\pi_{0} \circ \varrho_{K}, \mathscr{H}_{0}\right)$. Endomorphisms may be multiplied

$$
\varrho_{K} \varrho_{J}(A)=\varrho_{K}\left(\varrho_{J}(A)\right) \text { etc. }
$$

Therefore, $\pi_{0} \circ \varrho_{K} \varrho_{J}$ is also a positive energy representation realized in $\mathscr{H}_{0}$. It may be reducible. To reduce it into irreducible subrepresentations $\cong \pi_{0}{ }^{\circ} \varrho_{L}$, one needs 
projection operators $\Pi\left(\begin{array}{cc}J \\ L & K\end{array}\right)$ which commute with representation operators $\pi_{0} \circ \varrho_{K} \varrho_{J}(A)$,

$$
\varrho_{K} \varrho_{J}(A) \Pi\left(\begin{array}{cc}
J \\
L & K
\end{array}\right)=\Pi\left(\begin{array}{cc}
J \\
L & K
\end{array}\right) \varrho_{K} \varrho_{J}(A)
$$

and intertwining operators $T\left(\begin{array}{cc}J \\ L & K\end{array}\right)$ which establish the unitary equivalence of $\pi_{0} \circ \varrho_{K} \varrho_{J}$ restricted to $\Pi_{L} \mathscr{H}_{0}$ with $\pi_{0} \circ \varrho_{L}$,

$$
\begin{gathered}
\varrho_{L}(A) T\left(\begin{array}{cc}
J \\
L & K
\end{array}\right)=T\left(\begin{array}{cc}
J \\
L & K
\end{array}\right) \varrho_{K} \varrho_{J}(A), \\
T\left(\begin{array}{cc}
J \\
L & K
\end{array}\right)^{*} T\left(\begin{array}{cc}
J \\
L & K
\end{array}\right)=\Pi\left(\begin{array}{cc}
J \\
L & K
\end{array}\right) .
\end{gathered}
$$

Here we have omitted symbols $\pi_{0}$, it is understood that all operators act on $\mathscr{H}_{0}$, for the moment. Comparing with Eq. (1.21) and remembering that $\pi_{K}$ may be identified with $\pi_{0} \circ \varrho_{K}$, we see that intertwiners $\Gamma\left(\begin{array}{cc}J \\ L & K\end{array}\right)$ get identified with the intertwiners $\pi_{0}\left(T\left(\begin{array}{cc}J \\ L & K\end{array}\right)\right)$ that reduce the representation $\pi_{0} \circ \varrho_{K} \varrho_{L}$. In our model these intertwiners are unique (up to phase factors). We will find elements $T$ satisfying Eqs. (1.45) and (1.46) in the universal enveloping algebra $\mathscr{U}($ Lie $\mathscr{A})$ of a Lie algebra of observables which is contained in Lie $\mathscr{A}$, their action on $\mathscr{H}_{0}$ is then given by $\pi_{0}(T)$. In conclusion, operators $\Gamma\left(\begin{array}{cc}J & K\end{array}\right)$ are composed of elements of $\mathscr{U}($ Lie $\mathscr{A})$ and identification operators.

Writing $[\varrho]$ for the equivalence class of unitary representation $\pi_{0} \circ \varrho$, the decomposition into irreducibles comes out as follows:

\section{Theorem 4 (Fusion Rules).}

$$
\begin{gathered}
{\left[\varrho_{1 / 2}^{2}\right]=\left[\varrho_{0}\right]+\left[\varrho_{1}\right],} \\
{\left[\varrho_{1 / 2} \varrho_{1}\right]=\left[\varrho_{1} \varrho_{1 / 2}\right]=\left[\varrho_{1 / 2}\right],} \\
{\left[\varrho_{1}^{2}\right]=\left[\varrho_{0}\right]}
\end{gathered}
$$

and $\left[\varrho_{0} \varrho_{J}\right]=\left[\varrho_{J}\right]$ for $J=0, \frac{1}{2}, 1$.

It is not an accident that this recovers the fusion rules of the conformal field theory. Using the intertwiners as building blocks, field operators $\phi^{J}=\Gamma^{J} U$ can be constructed which act on the Hilbert space

$$
\mathscr{H}=\bigoplus_{J} \mathscr{H}^{J}
$$

which is a direct sum of superselection sectors, without multiplicity. Operators $\Gamma^{J}$ can be composed from intertwiners $\Gamma\left(\begin{array}{cc}J \\ L & K\end{array}\right)$, so that

$$
A \Gamma^{J}=\Gamma^{J} \varrho_{J}(A), \quad \Gamma^{J} \Gamma^{J *}=1 .
$$


The last property fixes complex factors, up to some phases without physical meaning.

We note that states of the form

$$
\phi^{L} \phi^{J}|0\rangle \in \mathscr{H}
$$

transform according to a representation $\cong \pi_{0} \circ \varrho_{L} \varrho_{J}$ (or a subrepresentation of it). This is because they are of the form $\Gamma^{L} \Gamma^{J}|\xi\rangle,|\xi\rangle \in \mathscr{H}_{0}$, and

$$
\pi(A) \Gamma^{L} \Gamma^{J}|\xi\rangle=\Gamma^{L} \Gamma^{J} \pi_{0} \circ \varrho_{L} \varrho_{J}(A)|\xi\rangle,
$$

by bosonization formula (1.15) and Eq. (1.51). The decomposition of representation $\pi_{0} \circ \varrho_{L} \varrho_{J}$ into irreducibles $\pi_{0} \circ \varrho_{K}$ will therefore recover the fusion rules of the conformal field theory.

To accommodate the quantum symmetry, one will need multiplets of field operators $[16,17]$. Our field operators will be given by a bosonization formula

$$
\phi_{m}^{J}=\Gamma_{m}^{J} U, \quad U \in \mathscr{A}, U U^{*}=1 .
$$

They are required to obey the same commutation relations with observables as the single component fields considered before:

$$
A \Gamma_{m}^{J}=\Gamma_{m}^{J} \varrho_{J}(A),
$$

so that

$$
A \phi_{m}^{J}=\phi_{m}^{J} \varrho(A), \quad \varrho \equiv \sigma_{U} \varrho_{J},
$$

independent of $m$.

Such field operators can be constructed from the intertwining operators $\Gamma\left(\begin{array}{cc}J \\ K & L\end{array}\right)$ between sectors and quantum group Clebsch Gordan coefficients. The operators $\Gamma_{m}^{J}$ must be linear combinations of intertwiners $\Gamma\left(\begin{array}{cc}J \\ L & K\end{array}\right)$, in order to have covariance Property 1 of Theorem 2, with coefficients proportional to quantum group Clebsch Gordan coefficients, in order to have quantum group covariance. Some overall factors $c_{J K L}$ remain free. The general idea is to require identity 5 of Theorem 2 ,

$$
\Gamma_{k}^{J} \Gamma_{l}^{J} \varepsilon_{J}=\sum_{m, n} \Gamma_{m}^{J} \Gamma_{n}^{J} \mathscr{R}_{k l}^{m n}(J)
$$

and reconstruct the quantum group as a commutant [14] of the matrix $\mathscr{R}$. Equation (1.56) is the generalization of a relation in the field algebra that is valid in Doplicher and Roberts theory in $d \geqq 3$ dimensions.

Consistency of Eq. (1.56) would require that the $\mathscr{R}$-matrix satisfies Yang Baxter equations, and has the same eigenvalues as $\varepsilon_{J}$.

The range of indices is not known a priori, and must be found together with the matrix $\mathscr{R}$. The braid group representation (1.29) determines a statistical dimension $d_{J}=d\left(\varrho_{J}\right)$. It is given by the index of a linear transformation which determines $\varrho_{J}$ in our model. This statistical dimension comes out as

$$
d_{J}=1, \sqrt{2}, 1 \text { for } J=0, \frac{1}{2}, 1
$$

and satisfies the same sum rules as quantum dimensions do in quantum group theory. This indicates that the quantum dimension substitutes for ordinary dimension in Doplicher Roberts theory when space time dimension $d \leqq 2$. 
From the knowledge of the endomorphisms one obtains also representations of the braid group by operators $\sigma_{n}=\varrho^{n-1}\left(\varepsilon_{J}\right)$ as explained before. This braid group representation is found explicitly and is related to a Temperley-Lieb-Jones algebra.

Let us fix $J$. The interesting case is $J=\frac{1}{2}$. The action of braid group generators $\sigma_{n}$ on products of chiral vertex operators $\Gamma(s)=\Gamma\left(\begin{array}{cc}J \\ L & K\end{array}\right)$ by right multiplication yields standard braid matrices $[8,18,19,20] R$. In particular

$$
\Gamma\left(s_{1}\right) \Gamma\left(s_{2}\right) \varepsilon_{J}=\sum_{s_{3} s_{4}} \Gamma\left(s_{3}\right) \Gamma\left(s_{4}\right) R_{s_{1} s_{2}}^{s_{3} s_{4}}(J) .
$$

Explicit calculation shows that they agree with the expressions for $R$-matrices in terms of $6 j$-symbols for $U_{q}(s l(2)), q=-i$, multiplied with an overall phase factor.

It is not an accident that our matrices $R_{s_{1} s_{2}}^{s_{3} s_{4}}$ as defined by Eq. (1.58) agree with the known $R$-matrices of the conformal field theory model. If $\phi(s)=\Gamma(s) U$ and $\phi^{\prime}(s)$ $=\Gamma(s) U^{\prime}$ are localized in disjoint intervals, then Eq. (1.58) implies local braid relations for them, as one has in conformal field theory

$$
\phi\left(s_{1}\right) \phi^{\prime}\left(s_{2}\right)=\sum_{s_{3} s_{4}} \phi^{\prime}\left(s_{3}\right) \phi\left(s_{4}\right) R_{s_{1} s_{2}}^{s_{3} s_{4}} .
$$

This is established in the same way as for Remark 3. Comparing Eqs. (1.56) and (1.58) it follows that the standard $R$-matrix $R_{s_{1} s_{2}}^{s_{3} s_{4}}$ and the quantum group invariant matrix $\mathscr{R}$ must be related by a "vertex-SOS-transformation" in the terminology of Fröhlich et al. [41]. In this formula, summation over intermediate states should only run over "physical" representations $S^{\prime}=0, \frac{1}{2}, 1$, excluding $\frac{3}{2}$. This creates the problem mentioned before and is responsible for the fact that we were only able to demonstrate the validity of local braid relations on a subspace of $\mathscr{H}_{\text {phys }}$ (Sect. 6).

As was mentioned above, the braid group generators $\sigma_{n}$ are expressed in terms of projectors $E_{n}$ of a Temperley-Lieb algebra

$$
\begin{gathered}
\sigma_{n}=z\left(q E_{n}-\left(1-E_{n}\right)\right), \\
E_{i} E_{i \pm 1} E_{i}=\frac{1}{Q} E_{i}, \quad E_{i} E_{j}=E_{j} E_{i} \text { for } \quad|i-j| \geqq 2
\end{gathered}
$$

with

$$
z=-e^{3 \pi i / 8}, \quad q=i, \quad \text { and } \quad Q \equiv d_{1 / 2}^{2}=\left[q+q^{-1}+2\right]=2 .
$$

This is isomorphic to the Temperley-Lieb algebra of the 2-state Potts model (Ising model on a 2-dimensional lattice) [21,22].

It turns out that the projectors in the Temperley-Lieb-Jones algebra generate the whole observable algebra $\pi_{0}(\mathrm{Lie} \overline{\mathscr{A}})$ in the vacuum sector. In this description, the endomorphism $\varrho_{1 / 2}$ takes a particular simple form, $\varrho_{1 / 2}\left(E_{n}\right)=E_{n+1}$.

Let $\mathscr{C}$ be another algebra which is obtained from the group algebra [23] $\mathbf{C} B_{\infty}$ of the braid group $B_{\infty}$ by imposing relations, such as quadratic, cubic or higher order equations which are to be satisfied by all braid group generators $\sigma_{n}$ [39]. If we wanted to start from such an algebra $\mathscr{C}$ as a candidate for vacuum representation of the global observable algebra, with its natural endomorphism, $\varrho\left(\sigma_{n}\right)=\sigma_{n+1}$, construction of a local quantum field theory would require that we enlarge $\mathscr{C}$ by adding limit points [in the topology furnished by a suitable state $\left.\omega_{0}(\cdot) \equiv\langle 0|\cdot| 0\rangle\right]$ and exhibit subalgebras $\mathscr{A}(I)$ which commute for relatively spacelike domains. An example of such a construction was described by Connes 
and Evans [29]. In general, the vacuum representation need not be faithful, but the knowledge of the local subalgebras permits to reconstruct the global observable algebra. The whole construction amounts to lifting the structure of space time as a causally oriented manifold to the algebra (cp. Sect. 2.3).

In our model, the algebra $\mathscr{C}$ gets lifted to

$$
\mathscr{C} \otimes \mathbf{C} \mathbf{Z}_{2} .
$$

The group $\mathbf{Z}_{2}$ has two elements 1 and $Y$ with $Y^{2}=\mathbf{1}$. They span the group algebra $\mathbf{C Z}_{2}$ and commute with generators $\sigma_{n}$ of $\mathscr{C}$. The fundamental endomorphism $\varrho_{1 / 2}$ acts on $\mathscr{C} \otimes \mathbf{C Z}_{2}$ according to

$$
\varrho_{1 / 2}\left(\sigma_{n} y\right)=\chi_{1 / 2}(y) \sigma_{n+1} y
$$

for $y \in \mathbf{Z}_{2} \cdot \chi_{1 / 2}$ is the nontrivial character on $\mathbf{Z}_{2}$, i.e. $\chi_{1 / 2}(\mathbf{1})=1$ and $\chi_{1 / 2}(Y)=-1$. The global field algebra includes additional generators $\Gamma_{a}^{1 / 2}$ with relations

$$
\sigma_{n} y \Gamma_{a}^{1 / 2}=\chi_{1 / 2}(y) \Gamma_{a}^{1 / 2} \sigma_{n+1} y,
$$

and quantum group generators which commute with $\sigma_{n}$ and $y \in \mathbf{Z}_{2}$ and which obey generalized commutation relations (6.30) with $\Gamma_{a}^{1 / 2}$. They invoke comultiplication (6.10).

These formulae are easy to generalize, but to generalize the embeddings of local subalgebras $\mathscr{A}(I)$ is a nontrivial task. Also the physical field algebra will not be a faithful representation of the algebra $\mathscr{F}$ defined by relations (1.63), (6.30) and relations in $\mathscr{C}$. A representation of the quantum group covariant field algebra $\mathscr{F}$ in an unphysical linear space $\mathscr{H}$ is furnished by the theory of induced representations. Let $\pi_{0}$ be the vacuum representation of $\mathscr{C} \otimes \mathbf{C Z}_{2}$ on Hilbert space $\mathscr{H}_{0}$ (obtained from state $\omega_{0}$ ), and define $\mathscr{H}$ to consist of $\mathbf{C}$-linear functions $f$ on $\mathscr{F}$ with values in $\mathscr{H}_{0}$ which obey covariance condition

$$
f(A \phi)=\pi_{0}(A) f(\phi) \text { for } \quad A \in \mathscr{C} \otimes \mathbf{C Z}, \quad \phi \in \mathscr{F} .
$$

$\mathscr{F}$ acts on $\mathscr{H}$ according to

$$
(\psi f)(\phi)=f(\phi \psi)
$$

\section{The Observable Algebra and its Positive Energy Representations}

2.1. The Virasoro Algebra with $c=\frac{1}{2}$ and Associated Net of Local Observables. Einstein causality plays a pivotal role in algebraic field theory. Therefore it is appropriate to start with some introductory remarks to show what becomes of it when we restrict attention to chiral observables.

2-dimensional conformal quantum field theory lives on a tube $[24,25]$ $\tilde{M}=\mathbf{R} \times \mathbf{S}^{1}$ with points $(\tau, \sigma), \tau=-\infty \ldots \infty, \sigma=0 \ldots 2 \pi$. This space time manifold $\tilde{M}$ contains Minkowski spaces $M_{\zeta}$ as subspaces (see Fig. 2). Their positions are fixed by the unique point $\zeta \in \tilde{M}$ at spacelike infinity of $M$. Manifold $\tilde{M}$ inherits from $M$ a global causal structure - i.e. a notion of positive timelike, spacelike, and negative timelike - which is invariant under the action of an infinite dimensional space time symmetry group (conformal group) [26]

$$
G=\left[\widehat{\operatorname{Diff}}\left(\mathbf{S}^{1}\right) \times \widehat{\operatorname{Diff}}\left(\mathbf{S}^{1}\right)\right] / \mathbf{Z}_{\mathrm{diag}}
$$




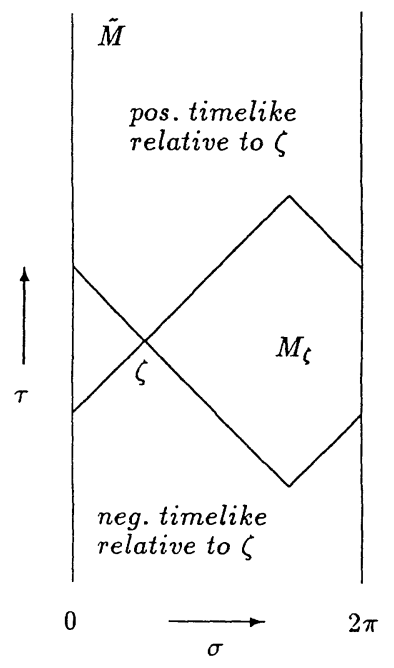

Fig. 2. Diffeomorphism invariant global causal structure on the tube $\tilde{M}=\mathbf{R} \times \mathbf{S}^{1} . M_{\zeta}$ is the Minkowski space with point $\zeta$ at "spacelike infinity". It consists of all points of $\tilde{M}$ which are relatively spacelike to $\zeta$

$\widehat{\operatorname{Diff}}\left(\mathbf{S}^{1}\right)$ is the universal covering group of Diff $\left(\mathbf{S}^{1}\right)$. Its elements are diffeomorphisms $f: \mathbf{R} \rightarrow \mathbf{R}$ with $f(\sigma+2 \pi)=f(\sigma)+2 \pi$. The first factor acts on $\sigma_{+}=\tau+\sigma$, and the second on $\sigma_{-}=\tau-\sigma$.

In conformal field theory, this symmetry is unitarily implemented, so that the Hilbert space of physical states carries a unitary representation of the central extension $G^{\sim}$ of $G$ (i.e. a ray representation of $G$ ). This symmetry group furnishes also the most basic observables.

The Lie algebra of $G^{\sim}$ is a direct sum $\operatorname{Vir} \oplus \operatorname{Vir}$ of two Virasoro algebras. They are generated by the two light cone components of the stress energy tensor

$$
T_{ \pm}\left(z_{ \pm}\right), \quad z_{ \pm}=e^{i \sigma_{ \pm}} .
$$

Because of $2 \pi$-periodicity in $\sigma$, they are 1 -valued functions of their argument $z_{ \pm} \in \mathbf{S}^{1}$.

We start from algebras of local observables which are generated by the stress tensor. Therefore the observables will depend on only one of the light cone variables $z_{ \pm}$, i.e. they live on the circle $\mathbf{S}^{1}$. Relatively spacelike domains $\mathcal{O}_{1}$ and $\mathcal{O}_{2}$ in $\tilde{M}$ project on disjoint intervals $I_{1}$ and $I_{2}$ on the circle. Therefore

$$
\text { relatively spacelike }=\text { disjoint } \text { on the circle } \mathbf{S}^{1} \text {, }
$$

and observables which are localized on disjoint intervals on $\mathbf{S}^{1}$ should commute, by Einstein causality.

We restrict attention to one of the algebras Vir, and drop suffix + or - . The stress tensor has explicitly known commutation relations

$$
[T(z), T(w)]=\left[2 \delta^{\prime}(z-w)+\delta(z-w) \frac{d}{d z}\right] T(z)+\frac{c}{12} \delta^{\prime \prime \prime}(z-w) .
$$

The commutator is nonvanishing only for coinciding arguments, in agreement with the above locality requirement. In this paper we consider the theory with 
central charge $c=\frac{1}{2}$, (i.e. the scaling limit of the 2-dimensional Ising model at the critical point),

$$
T(z)=\sum_{n \in \mathbf{Z}} L_{n} z^{-n-2} \text { with } L_{-n}=L_{n}^{*},
$$

and the Fourier components $L_{n}$ satisfy commutation relations of a Virasoro algebra. In a positive energy representation of Vir, the "conformal energy"

$$
L_{0} \geqq 0 \text {. }
$$

We define complex Lie algebras of observables Vir $(I)$ for arbitrary intervals $I \subset \mathbf{S}^{1}$. $\operatorname{Vir}(I)$ is spanned by operators

$$
T(f)=\frac{1}{2 \pi i} \oint_{\mathbf{S}^{1}} z d z T(z) f(z)
$$

with smooth functions $f$ having support

$$
\operatorname{supp} f \subset I \text {. }
$$

$T(f)$ are unbounded operators. Associative algebras of bounded operators are obtained as group algebra of a central extension $\operatorname{Diff}^{\sim}(I)$ of the Lie group

$$
\operatorname{Diff}(I) \subset \operatorname{Diff}\left(\mathbf{S}^{1}\right),
$$

which consists of diffeomorphisms of $\mathbf{S}^{1}$ which act trivially on the complement of $I$ in $\mathbf{S}^{\mathbf{1}}$.

The Virasoro algebra with central charge $c=\frac{1}{2}$ admits 3 inequivalent positive energy representations $\pi_{I}, I=0, \frac{1}{2}, 1$. They are lowest weight representations with weights

$$
\lambda_{0}=0, \quad \lambda_{1 / 2}=\frac{1}{16}, \quad \lambda_{1}=\frac{1}{2} .
$$

They act in the Hilbert spaces $\mathscr{H}_{I}$ with the lowest weight vector $\left|\lambda_{I}\right\rangle$

$$
\begin{gathered}
\pi_{I}\left(L_{n}\right)\left|\lambda_{I}\right\rangle=0 \text { for } n>0, \\
\pi_{I}\left(L_{0}\right)\left|\lambda_{I}\right\rangle=\lambda_{I}\left|\lambda_{I}\right\rangle .
\end{gathered}
$$

$\pi_{0}$ is the vacuum representation; it acts on the vacuum sector $\mathscr{H}_{0}$ which contains the vacuum vector $|0\rangle$. All the representations restrict to faithful representations of the local algebras $\operatorname{Vir}(I)$ and we may identify $\operatorname{Vir}(I)$ with its vacuum representation $\pi_{0}(\operatorname{Vir}(I))$.

We seek endomorphisms $\varrho_{J}$ of a net of algebras of observables $\mathscr{A}(I)$ such that $\pi_{J}=\pi_{0} \circ \varrho_{J}$. As discussed in the introduction, we can expect that they exist, if $\mathscr{A}(I)$ are von Neumann algebras and satisfy Haag duality. Haag duality means that the observable algebras are maximal, i.e. it is not possible to add additional local fields which act in $\mathscr{H}_{0}$. The net of algebras Diff $(I)$ does not have this property (see later). This is the reason why we need to work with a global Lie algebra Lie $\overline{\mathscr{A}} \supset$ Vir of observables that is larger than the Virasoro algebra. The weak closure of the associative algebras $\pi_{0}$ (Span $\left.\operatorname{Diff} \sim(I)\right)$ would satisfy Haag duality [27]. But it is inconvenient for practical calculations. We prefer to work with explicitly known Lie algebras of observables.

We will exhibit two global algebras Lie $\mathscr{A} \subset$ Lie $\overline{\mathscr{A}}$ of observables. The Virasoro generators are in Lie $\mathscr{A}$, but not in Lie $\mathscr{A}$. They are formal limits of elements in Lie $\mathscr{A}$, though. 
It will be shown that all the unitary positive energy representations of the Virasoro algebra with $c=\frac{1}{2}$ extend to unitary representations of Lie $\mathscr{A}$, and also of Lie $\bar{A}$. Suitable endomorphisms $\varrho_{J}$ are exhibited to obtain all of these representations $\pi_{J}$ from the vacuum representation $\pi_{0}$, i.e. $\pi_{J} \cong \pi_{0} \circ \varrho_{J}$. These endomorphisms are endomorphisms both of Lie $\mathscr{A}$ and of Lie $\overline{\mathscr{A}}$.

These endomorphisms are not localized. But localized endomorphisms (which generate the same representations) also exist and will be briefly described in Sect. 3.4. It would be important to show that these localized endomorphisms define endomorphisms of a Lie group with Lie algebra Lie $\overline{\mathscr{A}}$. Since this group would have to contain the diffeomorphisms in $\operatorname{Diff}^{\sim}\left(\mathbf{S}^{1}\right)$, it would follow that localized endomorphisms have the transportability property that was mentioned in the introduction.

2.2. Majorana Algebras on the Circle. We begin with the construction of local Lie algebras of observables Lie $\mathscr{A}(I)$. Its elements are constructed from bilinears in Majorana fields on the circle. They will be injected into a global algebra Lie $\mathscr{A}$ $C$ Lie $\overline{\mathscr{A}}$, and our endomorphisms shall be endomorphisms which are obtained by restriction of endomorphisms of a suitable Majorana algebra.

In the case of a topological nontrivial space time, such as $\tilde{M}=\mathbf{R} \times \mathbf{S}^{1}$ and its projection $\mathbf{S}^{1}$, the construction of a global algebra $\mathscr{A}$ is subtle. Whereas local algebras $\mathscr{A}(I)$ can be identified with their vacuum representation, the same is not true for $\mathscr{A}$. Two domains $I_{1}$ and $I_{2}$ may cover all of $\mathbf{S}^{1}$, therefore $\mathscr{A}$ may contain global quantities (e.g. exponentials of charge operators in Lie $\mathscr{A}$ ) which lie in the center of $\mathscr{A}$. These charges may have different values in different superselection sectors. They must not be identified with multiples of the identity. Therefore $\mathscr{A}$ will have a nontrivial center, and $\pi_{0}(\mathscr{A})$ is not a faithful representation. Familiar examples of such charges which label superselection sectors in the real world are electric charge $Q$ and fermionic charge $(-1)^{F}, F=$ number of fermions. In our example, the global algebra will be obtained from a "universal" Majorana algebra which contains a central element $Y$. It may take values \pm 1 in the irreducible representations. In principle it is possible to avoid the use of a global algebra altogether, by working with localized endomorphisms in the punctured circle. In practise this is inconvenient.

We denote points on the circle by $z=e^{i \phi}, \phi=0 \ldots 2 \pi$. The Majorana algebra on the circle is generated by field operators $\psi(z)$ which satisfy canonical anticommutation relations (CACR).

$$
\{\psi(z), \psi(w)\}=2 \pi i \delta(z-w) \mathbf{1}
$$

and hermiticity condition

$$
\psi(z)^{*}=z \psi(z) .
$$

More precisely, only smeared fields

$$
\psi(f)=\frac{1}{2 \pi i} \oint_{\mathbf{S}^{1}} z^{-1 / 2} d z f(z) \psi(z)
$$

are operators (hermitian for real $f$ ), and their anticommutation relations make sense for continuous sections $f$ of some line bundle over $\mathbf{S}^{1}$. These spaces of sections $\Gamma_{N s}$ and $\Gamma_{R}$ may be identified with functions on $\mathbf{R}$ which are $2 \pi$-antiperiodic respectively periodic. Accordingly, there are actually two Majorana algebras $\mathrm{Maj}_{\mathrm{NS}}$ and $\mathrm{Maj}_{\mathrm{R}}$, generated by fields with boundary conditions

$$
\psi\left(z e^{2 \pi i}\right)= \pm \psi(z) \quad+\text { for } \psi \in \mathrm{Maj}_{\mathrm{NS}},- \text { for } \psi \in \mathrm{Maj}_{\mathbf{R}} .
$$


The fields can be expanded in Fourier modes

$$
\psi(z)=\sum_{r \in \mathbf{Z}+1 / 2} b_{r} z^{-r-1 / 2} \in \mathrm{Maj}_{\mathrm{NS}}
$$

and

$$
\psi(z)=\sum_{n \in \mathbf{Z}} b_{n} z^{-n-1 / 2} \in \mathrm{Maj}_{\mathbf{R}} .
$$

The commutation relations and hermiticity conditions are in either case

$$
\left\{b_{a}, b_{c}\right\}=\delta_{a,-c} \mathbf{1}, \quad b_{a}^{*}=b_{-a},
$$

$a \in \mathbf{Z}+\frac{1}{2}$ for $b_{a} \in \mathrm{Maj}_{\mathrm{NS}}$, and $a \in \mathbf{Z}$ for $b_{a} \in \mathrm{Maj}_{\mathrm{R}}$. Each of the two algebras possesses one faithful *-representation generated by lowest weight vectors $|N S\rangle$ and $|R\rangle$.

$$
\begin{array}{ccc}
b_{r}|N S\rangle=0 \quad \text { for } \quad r \geqq \frac{1}{2}, & r \in \mathbf{Z}+\frac{1}{2}, \\
b_{n}|R\rangle=0 \quad \text { for } \quad n \geqq 1, \quad n \in \mathbf{Z} .
\end{array}
$$

Note that $b_{0}^{2}=\frac{1}{2}$ by CACR, and $b_{0}|R\rangle$ is also annihilated by all $b_{n}$ with $n \geqq 1$. In the literature, the algebra generated by $b_{0} b_{n}$ is sometimes called Majorana algebra in the R-sector. Its generators $b_{0} b_{n}$ obey canonical commutation relations. The representations of our fermionic algebra $\mathrm{Maj}_{\mathrm{R}}$ splits into two representations of this bosonic algebra.

We wish to exhibit both the NS-representation and the R-representation as representations of a single algebra Maj. Basically, this is done by admitting test functions $f \in \Gamma_{\mathrm{NS}} \oplus \Gamma_{\mathrm{R}}$. They are single valued continuous functions on the double cover $\widetilde{\mathbf{S}}^{1}$ of $\mathbf{S}^{1}$ with points $z^{1 / 2}=e^{i \phi / 2}, \phi=0 \ldots 4 \pi$. By abuse of notation we regard functions on $\widetilde{\mathbf{S}}^{1}$ as functions of $z$.

Definition 2.1. The universal Majorana algebra Maj is the associative *-algebra with identity which is generated by a central element $Y$ and smeared fields on $\widetilde{\mathbf{S}}^{1}$,

$$
\psi(f)=\frac{1}{4 \pi i} \oint_{\tilde{\mathbf{s}}^{1}} z^{-1 / 2} d z f(z) \psi(z),
$$

subject to anticommutation relations

$$
\begin{gathered}
\{\psi(z), \psi(w)\}=\pi i\left[\delta(z-w)-Y \delta\left(z-w e^{2 \pi i}\right)\right], \\
{[Y, \psi(z)]=0,}
\end{gathered}
$$

hermiticity condition

$$
\psi(z)^{*}=z \psi(z)
$$

and boundary conditions

$$
\psi\left(z e^{2 \pi i}\right)=-Y \psi(z), \quad Y^{2}=1 .
$$

The decomposition in Fourier modes reads

$$
\psi(z)=\sum_{a \in \frac{1}{2} \mathbf{Z}} b_{a} z^{-a-1 / 2} .
$$

The Fourier modes obey relations

$$
\begin{aligned}
& \left\{b_{a}, b_{c}\right\}=\frac{1}{2}\left[1+(-1)^{2 a} Y\right] \delta_{a,-c}, \\
& {\left[Y, b_{a}\right]=0, \quad Y b_{a}=(-1)^{2 a} b_{a} .}
\end{aligned}
$$


It follows that

$$
Y=4 b_{0}^{2}-1
$$

Therefore the algebra is actually generated by smeared fields $\psi(f)$ alone.

Proposition 2.2. Maj admits two inequivalent irreducible ${ }^{*}$-representations $\pi_{\mathrm{NS}}$ and $\pi_{\mathrm{R}}$ with lowest weight vectors $|\mathrm{NS}\rangle$ and $|\mathrm{R}\rangle$.

$$
\pi_{\mathrm{NS}}(Y)=-1, \pi_{\mathrm{NS}}\left(b_{n}\right)=0 \quad \text { for } \quad n \in \mathbf{Z}, \quad \mathrm{Maj} / \operatorname{ker} \pi_{\mathrm{NS}} \cong \mathrm{Maj}_{\mathrm{NS}}
$$

while

$$
\pi_{\mathrm{R}}(Y)=1, \pi_{\mathrm{R}}\left(b_{r}\right)=0 \quad \text { for } \quad r \in \mathbf{Z}+\frac{1}{2}, \quad \mathrm{Maj} / \operatorname{ker} \pi_{\mathrm{R}} \cong \mathrm{Maj}_{\mathbf{R}} .
$$

The proof is obvious. The universal Majorana algebra is only an auxiliary construct which will be used to build Lie algebras. In place of Maj we could work with $\mathrm{Maj}_{\mathrm{NS}} \oplus \mathrm{Maj}_{\mathrm{R}}$. This amounts to imposing the further relation $b_{a} b_{c}=0$ for $a+c \notin \mathbf{Z}$.

2.3. The Lie Algebra of Local Observables. The Lie algebra of observables shall be made of bilinears in the Majorana field. Their vacuum representation shall admit $|N S\rangle=|0\rangle$ as lowest weight vector. We wish to identify the local algebras Lie $\mathscr{A}(I)$ with their vacuum representation in accordance with the principles of the theory of superselection sectors. Therefore we will construct them out of fields in $\mathrm{Maj}_{\mathrm{NS}}$ and later inject them into a global algebra which is made out of fields in Maj.

Throughout the rest of this paper, $I \subset \mathbf{S}^{1}$ will be open intervals whose closure is not all of $\mathbf{S}^{\mathbf{1}}$.

Definition 2.1a. The real Lie algebra Lie $\mathscr{A}(I)$ is spanned by

1. the identity $\mathbf{1}$,

2. generators

$$
\int_{I} \int_{I} d z_{1} d z_{2} z_{1}^{-1 / 2} z_{2}^{-1 / 2} F\left(z_{1}, z_{2}\right) \psi\left(z_{1}\right) \psi\left(z_{2}\right),
$$

where $F$ is a real $C^{\infty}$ function on $\mathbf{S}^{1} \times \mathbf{S}^{1}$ with $F\left(z_{1}, z_{2}\right)=0$ unless $z_{1} \in I$ and $z_{2} \in I$, and $\psi(\cdot) \in \mathrm{Maj}_{\mathrm{NS}}$.

$F$ may be regarded as ordinary function because functions with the indicated support properties are identified in a natural way with elements of $\Gamma_{\mathrm{NS}}$.

By CACR of Majorana fields, Lie $\mathscr{A}(I)$ is indeed a Lie algebra. It will not contain the local Virasoro algebra, unless one proceeds to including limits or infinite sums of generators in Lie $\mathscr{A}(I)$. However, the stress tensor can be constructed as bilinear in the Majorana field.

Definition 2.3b. The real Lie algebra $\operatorname{Vir}(I)$ is spanned by identity 1 and

$$
T(f)=\oint_{\mathbf{S}^{1}} z d z f(z) T(z),
$$

where $f$ is a real $C^{\infty}$-function with $\operatorname{supp} f \subset I$, and

$$
T(z)=-\frac{1}{2} \lim _{w \rightarrow z}\left\{\psi(w) \frac{\partial}{\partial z} \psi(z)-(w-z)^{-2}\right\} .
$$

Elements of $\operatorname{Vir}(I)$ act as derivations of Lie $\mathscr{A}(I)$, and $\operatorname{Lie} \mathscr{A}(I) \oplus \operatorname{Vir}(I)$ is also a Lie algebra. From the CACR of Majorana fields one deduces also 
Proposition 2.4 (Locality). If $I \cap I^{\prime}=\emptyset$ then

$$
A \in \operatorname{Lie} \mathscr{A}(I) \oplus \operatorname{Vir}(I), \quad B \in \operatorname{Lie} \mathscr{A}\left(I^{\prime}\right) \oplus \operatorname{Vir}\left(I^{\prime}\right)
$$

implies $[A, B]=0$. In particular, the bilocal fields in Lie $\mathscr{A}(I)$ are relatively local to the stress tensor.

Next we define the global algebra Lie $\mathscr{A}$ to consist of bilinear in the Majorana fields $\psi(\cdot) \in$ Maj on the double covering $\widetilde{\mathbf{S}}^{1}$ of $\mathbf{S}^{1}$ which commute with rotations by $2 \pi$.

Definition 2.5. The real Lie algebra Lie $\mathscr{A}$ is generated by

1. the identity 1 .

2. generators

$$
\oint_{\tilde{\mathbf{S}}^{1}} \oint_{\tilde{\mathbf{S}}^{1}} d z_{1} d z_{2} z_{1}^{-1 / 2} z_{2}^{-1 / 2} F\left(z_{1}, z_{2}\right) \frac{1}{2}\left[\psi\left(z_{1}\right) \psi\left(z_{2}\right)+\psi\left(z_{1} e^{2 \pi i}\right) \psi\left(z_{2} e^{2 \pi i}\right)\right] .
$$

3. conformal energy and number operator

$$
\begin{gathered}
L_{0}=\sum_{r \in \frac{1}{2} \mathbf{Z}, r>0} r b_{-r} b_{r}+\frac{1}{8} b_{0}^{2}, \\
N_{0}=\sum_{r \in \frac{1}{2} \mathbf{Z}, r \geqq \frac{1}{2}} b_{-r} b_{r},
\end{gathered}
$$

where $b_{r}$ are the Fourier modes of $\psi$, viz.

$$
\psi(z)=\sum_{a \in \frac{1}{2} \mathbf{Z}} b_{a} z^{-a-1 / 2}
$$

and $F$ is a real $C^{\infty}$-function on $\widetilde{\mathbf{S}}^{1} \times \widetilde{\mathbf{S}}^{1}$.

We chose to include in the global algebra the generator $L_{0}$ of rotations of the circle, as is customary also in loop group theory [6], in order to define the notion of a positive energy representation by condition $L_{0} \geqq 0$.

Next we wish to inject the local algebras into the global one. An interval $I \subset \mathbf{S}^{1}$ is covered by two disjoint intervals $\widetilde{I} \subset \widetilde{\mathbf{S}}^{1}$ and $\widetilde{I} e^{2 \pi i} \subset \widetilde{\mathbf{S}}^{1}$ in the double covering $\widetilde{\mathbf{S}}^{1}$. The function $F$ in Definition 2.3 a may be regarded as function on $\widetilde{\mathbf{S}}^{1}$ with support on $\tilde{I} \times \tilde{I}$.

The algebras $\mathscr{A}(I)$ satisfy $\mathscr{A}(I) \subset \mathscr{A}(J)$ if $I \subset J$. One speaks of a "net of algebras." An injection $i$ of such a net of (Lie) algebras into a global algebra is given by a family of injective homomorphisms

$$
i_{I}: \mathscr{A}(I) \rightarrow \mathscr{A}
$$

such that $i_{I}(A)$ does not depend on $I$ so long as $A \in \mathscr{A}(I)$. We write $i$ for $i_{I}$ in this case.

Definition 2.6. An injection $i: \operatorname{Lie} \mathscr{A}(I) \rightarrow \operatorname{Lie} \mathscr{A}$ is defined by

$$
\begin{aligned}
i: & \int_{I} d z_{1} d z_{2} z_{1}^{-1 / 2} z_{2}^{-1 / 2} F\left(z_{1}, z_{2}\right) \psi_{\mathrm{NS}}\left(z_{1}\right) \psi_{\mathrm{NS}}\left(z_{2}\right) \\
& \left.\rightarrow \int_{\tilde{I} \tilde{I}} d z_{1} d z_{2} z_{1}^{-1 / 2} z_{2}^{-1 / 2} F\left(z_{1}, z_{2}\right) \frac{1}{2}\left[\psi\left(z_{1}\right) \psi\left(z_{2}\right)+\psi\left(z_{1} e^{2 \pi i}\right) \psi z_{2} e^{2 \pi i}\right)\right]
\end{aligned}
$$

and $i(\mathbf{1})=1$.

An index NS has been appended to $\psi_{\mathrm{NS}}$ to remind of the fact that elements of Lie $\mathscr{A}(I)$ were made from fields in $\mathrm{Maj}_{\mathrm{NS}}$. One must verify that $i$ is really an injection. Working out the commutation relations verifies that $i$ are homomor- 
phisms. The relation $Y \psi(z)=-\psi\left(z e^{2 \pi i}\right)$ is used to see this. Finally $i$ is obviously injective and independent of the choice of $I$.

Remark 2.7. The process of injection $i$ of local (Lie) algebras into a global algebra $\mathscr{A}=\mathscr{A}\left(\mathbf{S}^{1}\right)$ is like covering a manifold with charts. If we think of $I \subset \mathbf{S}^{1}$ as obtained by injecting subsets of a Euclidean space, then $i$ may be regarded as a lift of that injection to the algebra. We may obtain the subalgebras $i(\mathscr{A}(I))$ from subalgebras $\mathscr{A}\left(I_{1}\right)$ for intervals $I_{1} \subset \mathbf{S}^{1} \backslash\{\zeta\}$ in a fixed "Minkowski space" (see Sect. 2.1) by making use of the 1-parameter group of automorphisms $\alpha_{t}$ of $\mathscr{A}$ which is generated by $L_{0}$. If $I$ is obtained from $I_{1} \subset \mathbf{S}^{1} \backslash\{\zeta\}$ through rotation by $t$, then

$$
i(\mathscr{A}(I))=\alpha_{t}\left(i\left(\mathscr{A}\left(I_{1}\right)\right)\right) \text {. }
$$

But let us point out that the intersection of commutants

$$
\bigcap_{I^{\prime}, I^{\prime} \cap I=\emptyset} i(\mathscr{A}(I))
$$

in $\mathscr{A}$ is not precisely $i(\mathscr{A}(I))$ but includes also the element $Y$ in the center of $\mathscr{A}$. Injection $i$ will extend to an injection of extended algebras $\operatorname{Lie} \mathscr{A}(I) \oplus \operatorname{Vir}(I)$, which contain the local Virasoro algebras, into a suitable global Lie algebra Lie $\overline{\mathscr{A}} \supseteqq \mathrm{Lie} \mathscr{A} \oplus$ Vir. The stress tensor can be regarded as formal limit of elements in Lie $\mathscr{A}(I)$. This tells us that the injected stress tensor is

$$
T(z)=-\frac{1}{2} \lim _{w \rightarrow z}\left\{\frac{1}{2} \psi(w) \psi^{\prime}(z)+\frac{1}{2} \psi\left(w e^{2 \pi i}\right) \psi^{\prime}\left(z e^{2 \pi i}\right)-\frac{1}{(w-z)^{2}}\right\} .
$$

The injection $i$ extends to $i: \operatorname{Lie} \mathscr{A}(I) \oplus \operatorname{Vir}(I) \rightarrow \operatorname{Lie} \mathscr{A} \oplus \operatorname{Vir}$.

Lie $\mathscr{A} \oplus$ Vir is not suitable as a global Lie algebra, however, because it is not mapped into itself by endomorphisms which we are going to exhibit. We proceed to define a larger Lie algebra Lie $\overline{\mathscr{A}} \supset$ Lie $\mathscr{A} \oplus \mathrm{Vir}$, with central elements $1, Y$ and further generators

$$
\begin{gathered}
L_{n}, N_{n} \quad(n \in \mathbf{Z}), \\
J_{a b}=-J_{b a} \quad\left(a, b \in \frac{1}{2} \mathbf{Z}, a-b \in \mathbf{Z} \backslash\{0\}\right) .
\end{gathered}
$$

Hermitian conjugation acts as

$$
L_{n}^{*}=L_{-n}, \quad N_{n}^{*}=N_{-n}, \quad J_{a b}^{*}=J_{-b-a} .
$$

Definition (2.38) of the stress tensor yields Virasoro generators

$$
L_{n}=\sum_{c>n / 2}\left(c-\frac{n}{2}\right) b_{n-c} b_{c}+\frac{1}{8} \delta_{n, 0} b_{0}^{2} \text { for } n \geqq 0 .
$$

The other generators are defined as

$$
\begin{gathered}
N_{n}=\sum_{c>n} b_{n-c} b_{c} \text { for } n \geqq 0, \\
J_{a c}=b_{a} b_{c}-\frac{1}{4}\left[1+(-1)^{2 a} Y\right] \delta_{a,-c} .
\end{gathered}
$$

Summations run over $c \in \frac{1}{2} \mathbf{Z}$ (integers and half integers), $>$ means "strictly bigger." Generators $L_{n}$ and $N_{n}$ are exhibited in manifestly normal ordered form. 
It is straightforward to work out the commutation relations. They come out in the form

$$
\left[L_{n}, L_{k}\right]=(n-k) L_{n+k}+\ldots, \quad\left[L_{n}, N_{k}\right]=-k N_{n+k}+\ldots, \quad\left[N_{n}, N_{k}\right]=\ldots,
$$

where ... stands for finite linear combinations of generators $J_{a b}, \mathbf{1}$ and $Y$. More explicitly we have

Proposition 2.8. Generators $L_{n}, N_{n}, J_{a b}$ satisfy commutation relations

$$
\begin{aligned}
{\left[L_{n}, L_{m}\right]=} & (n-m) L_{n+m}+\frac{1}{24} n\left(n^{2}-1\right) \delta_{n,-m}, \\
{\left[L_{n}, N_{k}\right]=} & -k N_{n+k}+\sum_{\max (0, n+k)<a \leqq n}\left(a-\frac{n}{2}\right) \hat{J}_{n+k-a, a} \\
& +\sum_{\frac{n}{2}<a<\min (n, k+n)}\left(a-\frac{n}{2}\right) \hat{J}_{n+k-a, a} \\
& +\sum_{\max \left(0, \frac{n}{2}+k\right)<a \leqq n+k}\left(a-k-\frac{n}{2}\right) \hat{J}_{n+k-a, a},
\end{aligned}
$$

for $n \geqq 0$,

$$
\left[N_{k}, N_{l}\right]=\sum_{\max (0, l+k) \leqq c \leqq k} \hat{J}_{k+l-c, c},
$$

for $k \geqq 0$,

$$
\begin{aligned}
{\left[J_{a b}, L_{n}\right]=} & \left(\frac{n}{2}+b\right) \chi_{\frac{n}{2}+b} \hat{J}_{a, n+b}-\left(\frac{n}{2}+a\right) \chi_{\frac{n}{2}+a} \hat{J}_{b, n+a} \\
& +\left(-b-\frac{n}{2}\right) \chi_{-\frac{n}{2}-b} \hat{J}_{n+b, a}-\left(-a-\frac{n}{2}\right) \chi_{-\frac{n}{2}-a} \hat{J}_{n+a, b},
\end{aligned}
$$

for $n \geqq 0$,

$$
\left[J_{a b}, N_{k}\right]=\chi_{b} \hat{J}_{a, k+b}-\chi_{a} \widehat{J}_{b, k+a}-\chi_{-k-a} \hat{J}_{k+a, b}+\chi_{-k-b} \widehat{J}_{k+b, a},
$$

for $k \geqq 0$, and

$$
\begin{aligned}
{\left[J_{a b}, J_{c d}\right]=} & -\delta_{a,-c} J_{b d}+\delta_{b,-c} J_{a d}+\delta_{b,-d} J_{c a}-\delta_{a,-d} J_{c b} \\
& +\frac{1}{4}\left[1+(-1)^{2 a} Y\right]\left(-\delta_{a,-c} \delta_{b,-d}+\delta_{b,-c} \delta_{a,-d}\right. \\
& \left.+\delta_{b,-d} \delta_{a,-c}-\delta_{a,-d} \delta_{b,-c}\right) .
\end{aligned}
$$

We used the abbreviations

$$
\begin{gathered}
\hat{J}_{a b}=J_{a b}+\frac{1}{4}\left[1+(-1)^{2 a} Y\right] \delta_{a,-c}, \\
\chi_{a}= \begin{cases}1 & a>0 \\
0 & a \leqq 0\end{cases}
\end{gathered}
$$

We see that the commutation relations close. Therefore, finite linear combinations of the generators form a Lie algebra. It does not contain $i(\operatorname{Lie} \mathscr{A}(I) \oplus \operatorname{Vir}(I))$ as a subalgebra, though, because elements of $\operatorname{Vir}(I)$ are infinite sums of Virasoro generators with coefficients that decrease fast,

$$
T(f)=\sum \alpha_{-n} L_{n}, \quad \sum_{n}\left|\alpha_{n}\right||n|^{M}<\infty \quad \text { for all } \quad M>0 .
$$


This is easily rectified. We admit infinite sums of generators $1, Y, J_{a b}, N_{k}, L_{n}$ with coefficients that decrease fast with $|a|,|b|,|k|,|n|$. It follows from Proposition 2.8 that their commutation relations close. In this way, we obtain the desired Lie algebra Lie $\overline{\mathscr{A}} \supset$ Lie $\mathscr{A} \oplus$ Vir.

2.4. Decomposition of Majorana Representations into Representations of Observable Algebras. Since observables and Virasoro generators were made from Majorana fields, the two representations of Maj with lowest weight $|N S\rangle$ and $|R\rangle$ restrict to representations of Lie $\overline{\mathscr{A}}$. They are positive energy representations, but reducible.

The representation space $\mathscr{H}_{\mathrm{NS}}$ is spanned by vectors

$$
b_{-r_{N}} \ldots b_{-r_{1}}|\mathrm{NS}\rangle, \quad r_{i} \in \mathbf{Z}+\frac{1}{2}, \quad r_{N}>r_{N-1}>\ldots>r_{1} \geqq \frac{1}{2} .
$$

The complex Lie algebra $\pi_{\mathrm{Ns}}(\mathrm{Lie} \mathscr{A})$ is generated by elements $b_{r} b_{s}$ with $r, s \in \mathbf{Z}+\frac{1}{2}$. The other generators $X$ of $\pi_{\mathrm{Ns}}($ Lie $\overline{\mathscr{A}})$, in particular those of $\pi_{\mathrm{Ns}}(\mathrm{Vir})$, consist of infinite sums of such generators, but only a finite number of summands does not vanish when $X$ is applied to a basis vector. Therefore, $\mathscr{H}_{\mathrm{NS}}$ decomposes into two invariant subspaces $\mathscr{H}_{\mathrm{NS}}^{\text {even }} \oplus \mathscr{H}_{\mathrm{NS}}^{\text {odd }}$ for Lie $\overline{\mathscr{A}}$. They are spanned by vectors of the form (2.52) with $N$ even and odd respectively. They admit lowest weight vectors for the Virasoro algebra

$$
|\mathrm{NS}\rangle=|0\rangle \in \mathscr{H}_{\mathrm{NS}}^{\text {even }}, \quad L_{0}|0\rangle=0
$$

and

$$
b_{1 / 2}|\mathrm{NS}\rangle=\left|\frac{1}{2}\right\rangle \in \mathscr{H}_{\mathrm{NS}}^{\text {odd }}, \quad L_{0}\left|\frac{1}{2}\right\rangle=\frac{1}{2}\left|\frac{1}{2}\right\rangle .
$$

It is easy to see that there exist no further lowest weight vectors for Vir. Vectors (2.52) are eigenvectors of $L_{0}$ to eigenvalue $-\sum_{i} r_{i}$. For a lowest weight vector, this eigenvalue would have to equal $0, \frac{1}{16}$, or $\frac{1}{2}$. This admits only $N=0$ and $N=1, r_{1}=\frac{1}{2}$. Therefore the representation spaces $\mathscr{H}_{\mathrm{NS}}^{\text {even }}$ and $\mathscr{H}_{\mathrm{NS}}^{\text {odd }}$ are irreducible ones for Vir and for Lie $\overline{\mathscr{A}} \supset$ Vir. It is obvious that they remain irreducible when restricted to Lie $\mathscr{A}$. As representations of Vir they are uniquely characterized by their lowest weights $\lambda=0, \frac{1}{2}$. Similarly, $\mathscr{H}_{\mathrm{R}}$ is spanned by vectors

$$
b_{-n_{N}} \ldots b_{-n_{1}}|R\rangle, \quad n_{i} \in \mathbf{Z}, \quad n_{N}>n_{N-1}>\ldots>n_{1} \geqq \frac{1}{2} .
$$

The complex Lie algebra $\pi_{\mathrm{R}}($ Lie $\mathscr{A})$ is generated by elements $b_{m} b_{n}$ with $m, n \in \mathbf{Z}$ etc. One finds that $\mathscr{H}_{\mathrm{R}}$ decomposes into two equivalent representation spaces $\mathscr{H}_{\mathrm{R}}^{\text {even }} \oplus \mathscr{H}_{\mathrm{R}}^{\text {odd }}$ for Lie $\overline{\mathscr{A}}$ with lowest weight vectors

$$
|\mathrm{R}\rangle=\left|\frac{1}{16}\right\rangle \in \mathscr{H}_{\mathbf{R}}^{\text {even }}, \quad L_{0}\left|\frac{1}{16}\right\rangle=\frac{1}{16}\left|\frac{1}{16}\right\rangle
$$

and

$$
b_{0}|\mathbf{R}\rangle=\left|\frac{1}{16^{\prime}}\right\rangle \in \mathscr{H}_{\mathbf{R}}^{\text {odd }}, \quad L_{0}\left|\frac{1}{16^{\prime}}\right\rangle=\frac{1}{16}\left|\frac{1}{16^{\prime}}\right\rangle .
$$

We have proven

Proposition 2.9. The irreducible positive energy representations $\pi_{J}$ of the Virasoro algebra with central charge $c=\frac{1}{2}$ and lowest weight $\lambda_{J}=0, \frac{1}{16}, \frac{1}{2}$ extend to positive energy representations of Lie $\overline{\mathscr{A}}$ in the same representation space. 


\section{Endomorphisms Which Intertwine Positive Energy Representations}

3.1. Endomorphisms of the Universal Majorana Algebra which Restrict to Endomorphisms of the Lie Algebra of Observables Lie $\bar{A}$

Proposition 3.1. Define the action of $\varrho_{J}\left(J=0, \frac{1}{2}, 1\right)$ on generators $Y, b_{a}\left(a \in \frac{1}{2} \mathbf{Z}\right)$ of the universal Majorana algebra Maj by $\varrho_{J}(\mathbf{1})=\mathbf{1}$ and

$$
\begin{gathered}
\varrho_{1 / 2}\left(b_{a}\right)=\left\{\begin{array}{ll}
i b_{a+1 / 2}, & \varrho_{0}=i d, \\
\frac{i}{\sqrt{2}}\left(b_{1 / 2}-b_{-1 / 2}\right), & a=0 \\
-i b_{a-1 / 2}, & a \leqq-\frac{1}{2}
\end{array}, \quad \varrho(Y)=-Y,\right. \\
\varrho_{1}\left(b_{a}\right)=\left\{\begin{array}{ll}
-b_{a}, & a \neq 0, \pm \frac{1}{2} \\
b_{-a}, & a=0, \pm \frac{1}{2}
\end{array}, \quad \varrho(Y)=Y .\right.
\end{gathered}
$$

This defines *-endomorphisms of the *-algebra Maj. $\varrho_{1}$ is an automorphism but $\varrho_{1 / 2}$ is not.

Proof. It is easily verified that the relations in Maj are preserved, and $\varrho_{I}\left(b_{a}^{*}\right)$ $=\varrho_{I}\left(b_{-a}\right)=\varrho_{I}\left(b_{a}\right)^{*}$. Therefore $\varrho_{I}$ are $*$-endomorphisms. The other statements are also obvious. $\varrho_{1 / 2}$ is not an automorphism because $b_{1 / 2}+b_{-1 / 2} \notin \varrho_{1 / 2}$ (Maj) and $b_{0} \notin \varrho_{1 / 2}(\mathrm{Maj})$.

Remark 3.2. i) The automorphism $\varrho_{1}$ projects to an inner automorphism of $\mathrm{Maj}_{\mathrm{NS}}$ and of $\mathrm{Maj}_{\mathbf{R}}$. Explicitly

with unitaries $U$

$$
\varrho_{1}\left(b_{a}\right)=U b_{a} U^{*}
$$

$$
\begin{gathered}
U=\left(b_{1 / 2}+b_{-1 / 2}\right) \text { for } a \in \mathbf{Z}+\frac{1}{2}, \quad b_{a} \in \mathrm{Maj}_{\mathrm{NS}}, \\
U=b_{0} \sqrt{2} \text { for } a \in \mathbf{Z}, \quad b_{a} \in \mathrm{Maj}_{\mathrm{R}} .
\end{gathered}
$$

But the induced automorphism $\varrho_{1}$ of Lie $\mathscr{A}$ is not inner. It is conjugation by a reflection in the group of orthogonal transformations of $\Gamma_{\mathrm{NS}}$ or $\Gamma_{\mathrm{R}}$ which possess a determinant equal to 1 .

ii) The endomorphism $\varrho_{1 / 2}$ of Maj projects to homomorphisms $\mathrm{Maj}_{\mathrm{NS}} \rightarrow \mathrm{Maj}_{\mathrm{R}}$ and $\mathrm{Maj}_{\mathrm{R}} \rightarrow \mathrm{Maj}_{\mathrm{NS}}$.

Proposition 3.3. The endomorphisms of Maj of Proposition 3.1 induce endomorphisms of the global observable algebra Lie $\mathscr{A}$ and Lie $\bar{A}$. They restrict to a Cartan subalgebra spanned by commuting generators $L_{0}, N_{0}, H_{a}=b_{-1 / 2} b_{1 / 2}=J_{-1 / 2,1 / 2}$ $+\frac{1}{4}[1-Y]\left(a=\frac{1}{2}, 1, \frac{3}{2}, \ldots\right), 1$, and $Y$ as follows:

$$
\begin{gathered}
\varrho_{1 / 2}\left(L_{0}\right)=L_{0}-\frac{1}{2} N_{0}-\frac{1}{16} Y, \\
\varrho_{1 / 2}\left(N_{0}\right)=N_{0}-H_{1 / 2}, \\
\varrho_{1 / 2}\left(H_{a}\right)=H_{a+1 / 2}, \\
\varrho_{1}\left(L_{0}\right)=L_{0}+\frac{1}{2}-H_{1 / 2}-\frac{1}{4}[1+Y], \\
\varrho_{1}\left(N_{0}\right)=N_{0}+1-2 H_{1 / 2}-\frac{1}{2}[1+Y], \\
\varrho_{1}\left(H_{1 / 2}\right)=1-H_{1 / 2}-\frac{1}{2}[1+Y], \quad \varrho_{1}\left(H_{a}\right)=H_{a} \text { for } a>\frac{1}{2},
\end{gathered}
$$


and $\varrho_{1 / 2}(Y)=-Y, \varrho_{1}(Y)=Y$. The generators of the Cartan subalgebra act on the vacuum according to

$$
L_{0}|0\rangle=0, \quad N_{0}|0\rangle=0, \quad H_{a}|0\rangle=0, \quad[1+Y]|0\rangle=0 .
$$

$\varrho_{1}$ is an automorphism but $\varrho_{1 / 2}$ is not.

The action of endomorphism $\varrho_{1 / 2}$ on generators $L_{n}$ and $N_{n}$ is of the form

$$
\varrho_{1 / 2}\left(L_{n}\right)=L_{n}-\frac{1}{2} N_{n}+\ldots, \quad \varrho_{1 / 2}\left(N_{n}\right)=N_{n}+\ldots,
$$

where the dots stand for finite sums of generators $J_{a b}, \mathbf{1}$, and $Y$. This shows that $\varrho_{1 / 2}$ is lift of an automorphism of the algebra of vector fields and functions on the circle.

Proof. The complexified Lie algebra Lie $\mathscr{A}$ is generated by $b_{a} b_{c}\left(a, c \in \frac{1}{2} \mathbf{Z}, a-c \in \mathbf{Z}\right)$, $L_{0}$ and $N_{0}$. This includes $H_{a}=b_{-a} b_{a}$. Straightforward computation shows that each of these generators gets mapped into a finite sum of such generators. Our endomorphisms $\varrho_{J}$ extend to the unbounded generators $L_{n}, N_{n}$ in Lie $\bar{A}$ which are infinite sums of $b_{a} b_{c}$-terms. In particular the above formulae for $\varrho_{J}\left(L_{0}\right), \varrho_{J}\left(N_{0}\right)$, and $\varrho_{J}\left(H_{a}\right)$ hold true. Consider for instance $\varrho_{1 / 2}\left(L_{0}\right)$. Inserting the equality $4 b_{0}^{2}=1+Y$ in the definition of $L_{0}$ we find

$$
\begin{aligned}
\varrho_{1 / 2}\left(L_{0}\right) & =\sum_{c \geqq 1 / 2} c \varrho_{1 / 2}\left(b_{-c}\right) \varrho_{1 / 2}\left(b_{c}\right)+\frac{1}{32} \varrho_{1 / 2}(1+Y) \\
& =\sum_{c \geqq 1 / 2} c b_{-c-1} b_{c+1 / 2}+\frac{1}{32}[1-Y] \\
& =\sum_{a \geqq 1}\left(a-\frac{1}{2}\right) b_{-a} b_{a}+\frac{1}{32}[1-Y]=L_{0}-\frac{1}{2} N_{0}-\frac{1}{16} Y .
\end{aligned}
$$

No infinite constants arise because all terms in the various sums are manifestly in normal ordered form.

$\varrho_{1 / 2}$ is not an automorphism, because

$$
\begin{gathered}
b_{r}\left(b_{1 / 2}+b_{-1 / 2}\right) \notin \varrho_{1 / 2}(\operatorname{Lie} \overline{\mathscr{A}}) \text { for } r \in \mathbf{Z}+\frac{1}{2}, \\
b_{n} b_{0} \notin \varrho_{1 / 2}(\operatorname{Lie} \overline{\mathscr{A}}) \text { for } n \in \mathbf{Z} .
\end{gathered}
$$

3.2. Action of Endomorphisms on Positive Energy Representations. Now we will study representations $\pi_{0}{ }^{\circ} \varrho_{J}$ of the observable Lie algebra. They act in the same Hilbert space $\mathscr{H}_{0}$. We begin with the representations of the universal Majorana algebra.

Lemma 3.4. The representations of Maj obey

$$
\begin{aligned}
& \pi_{\mathrm{NS}} \circ \varrho_{1 / 2} \cong \pi_{\mathrm{R}}, \\
& \pi_{\mathrm{NS}} \circ \varrho_{1} \cong \pi_{\mathrm{NS}},
\end{aligned}
$$

and the lowest weight vector $|\mathrm{NS}\rangle$ of $\pi_{\mathrm{NS}}$ is also lowest weight vector of $\pi_{\mathrm{NS}}{ }^{\circ} \varrho_{1 / 2}$, i.e.

$$
\pi_{\mathrm{NS}} \varrho_{1 / 2}\left(b_{a}\right)|\mathrm{NS}\rangle=0 \text { for } a>0 \text {. }
$$

Proof. $\pi_{\mathrm{NS}} \circ \varrho_{1 / 2}\left(b_{a}\right)|\mathrm{NS}\rangle=i \pi_{\mathrm{NS}}\left(b_{a+1 / 2}\right)|\mathrm{NS}\rangle=0$ for $a<0$ since $\pi_{\mathrm{NS}}\left(b_{n}\right)=0$ for $n \in \mathbf{Z}$, and $\pi_{\mathrm{NS}}\left(b_{r}\right)|\mathrm{NS}\rangle=0$ for $r \in \mathbf{Z}+\frac{1}{2}, r>0$.

$$
\pi_{\mathrm{NS}} \circ \varrho_{1 / 2}(Y)=\pi_{\mathrm{NS}}(-Y)=-1=\pi_{\mathrm{R}}(Y) .
$$

This proves the last assertion and establishes $\pi_{\mathrm{NS}}{ }^{\circ} \varrho_{1 / 2}(\mathrm{Maj}) \cong \mathrm{Maj}_{\mathrm{R}}$, implying the first assertion. The second assertion follows from the fact that $\varrho_{1}$ projects to an inner automorphism of $\mathrm{Maj}_{\mathrm{NS}}$. 
It is instructive to recompute the two point function of the Majorana field in the R-representation. We write $\psi$ for $\pi_{\mathrm{NS}}(\psi)$ or $\pi_{\mathrm{R}}(\psi)$ when it acts on vectors in $\mathscr{H}_{\mathrm{NS}}$ and $\mathscr{H}_{\mathrm{R}}$ respectively,

$$
\begin{aligned}
& \langle\mathrm{R}|\psi(z) \psi(w)| \mathrm{R}\rangle=\left\langle\mathrm{NS}\left|\varrho_{1 / 2}(\psi(z)) \varrho_{1 / 2}(\psi(w))\right| \mathrm{NS}\right\rangle \\
& =\sum_{a, c \in \frac{1}{2} \mathbf{Z}} z^{-a-1 / 2} w^{-a-1 / 2}\left\langle\mathrm{NS}\left|\varrho_{1 / 2}\left(b_{a}\right) \varrho_{1 / 2}\left(b_{c}\right)\right| \mathrm{NS}\right\rangle \\
& =\sum_{0<a \in \mathbf{Z}} \sum_{0>c \in \mathbf{Z}} z^{-a-1 / 2} w^{-c-1 / 2}\left\langle\mathrm{NS}\left|b_{a+1 / 2} b_{c-1 / 2}\right| \mathrm{NS}\right\rangle \\
& \quad-\frac{1}{2} z^{-1 / 2} w^{-1 / 2}\left\langle\mathrm{NS}\left|\left(b_{1 / 2}-b_{-1 / 2}\right)^{2}\right| \mathrm{NS}\right\rangle .
\end{aligned}
$$

The lowest weight property of $|\mathrm{NS}\rangle$ was used, and terms $\left\langle\mathrm{NS}\left|b_{a+1 / 2} b_{ \pm 1 / 2}\right| \mathrm{NS}\right\rangle$ with $a>0$ and their conjugate were omitted because they give zero. Using CACR in the NS-representation to move creation operators $b_{a}$ with $a<0$ to the left and annihilation operators $b_{a}$ with $a>0$ to the right, one finds

$$
=\sum_{0<n \in \mathbf{Z}} z^{-n-1 / 2} w^{n-1 / 2}+\frac{1}{2} z^{-1 / 2} w^{-1 / 2} \text {. }
$$

This sums to

$$
\langle\mathbf{R}|\psi(z) \psi(w)| \mathbf{R}\rangle=\frac{1}{2}(z-w)^{-1}\left(\sqrt{\frac{z}{w}}+\sqrt{\frac{w}{z}}\right) .
$$

All formulae are to be understood in the sense of generalized functions, (i.e. smeared with test functions), $(z-w)^{-1}$ is the generalized function which is the limit of the holomorphic function $(z-w)^{-1}$ in $|z|>0,|w|<0$ [28].

Now we are already for the first main result

Theorem 3.5. The representation of Lie $\overline{\mathscr{A}} \supset$ Vir obey

1. $\pi_{0} \circ \varrho_{I} \cong \pi_{I}\left(I=0, \frac{1}{2}, 1\right)$.

2. the Virasoro lowest weight vector $|0\rangle \in \mathscr{H}_{0}$ is also lowest weight vector for the representations $\pi_{0} \circ \varrho_{I}$, viz.

$$
\begin{gathered}
\pi_{0} \circ \varrho_{I}\left(L_{n}\right)|0\rangle=0 \text { for } n>0, \\
\pi_{0} \circ \varrho_{I}\left(L_{0}\right)|0\rangle=\lambda_{I}|0\rangle
\end{gathered}
$$

with $\lambda_{I}=0, \frac{1}{16}, \frac{1}{2}$ for $I=0, \frac{1}{2}, 1$.

3. $\left\langle\lambda_{J}\left|\pi_{i}(A)\right| \lambda_{J}\right\rangle=\left\langle 0\left|\pi_{0}\left(\varrho_{J}(A)\right)\right| 0\right\rangle$ for elements $A$ of the universal enveloping algebra of Lie $\overline{\mathscr{A}}$.

Theorem 1 follows, since the injection $i$ was already constructed in Sect. 2.

Proof. For $I=0$ the assertions are trivial.

Consider $I=\frac{1}{2}$. The representations of the Majorana algebra restrict to Lie $\overline{\mathscr{A}}$ according to $\pi_{\mathrm{NS}} \cong \pi_{0} \oplus \pi_{1}$ and $\pi_{\mathrm{R}} \cong \pi_{1 / 2} \oplus \pi_{1 / 2}$ by the results of Sect. 2.4. Therefore Lemma 3.4 implies

$$
\pi_{0} \circ \varrho_{1 / 2} \oplus \pi_{1} \circ \varrho_{1 / 2} \cong \pi_{1 / 2} \oplus \pi_{1 / 2} .
$$

$\pi_{I} \circ \varrho_{1 / 2} \neq 0$ since $\pi_{I} \circ \varrho_{1 / 2}(\mathbf{1})=\mathbf{1}$. Therefore

$$
\pi_{0} \circ \varrho_{1 / 2} \cong \pi_{1 / 2} \text { and } \pi_{1} \circ \varrho_{1 / 2} \cong \pi_{1 / 2} .
$$

This proves 1 for $I=\frac{1}{2}$. Consider now

$$
\pi_{0} \circ \varrho_{1 / 2}\left(L_{0}\right)|0\rangle=\left(L_{0}-\frac{1}{2} N_{0}+\frac{1}{16}\right)|0\rangle=\frac{1}{16}|0\rangle,
$$


since $\pi_{0}(Y)=-1$. Since $\varrho_{1 / 2}$ is an endomorphism, $\varrho_{1 / 2}\left(L_{n}\right)$ satisfy the Virasoro commutation relations ${ }^{2}$. It follows that $\varrho_{1 / 2}\left(L_{n}\right)$ carries eigenvector $|0\rangle$ of $\varrho_{1 / 2}\left(L_{0}\right)$ with eigenvalue $\frac{1}{16}$ into an eigenvector with eigenvalue $\frac{1}{16}-n$. In the proof of Proposition 3.3 we computed

$$
\varrho_{1 / 2}\left(L_{0}\right)=\sum_{a \geqq 1}\left(a-\frac{1}{2}\right) b_{-a} b_{a}+\frac{1}{32}[1-Y],
$$

hence $\varrho_{1 / 2}\left(L_{0}\right) \geqq 0$. Therefore, nonzero eigenvectors of $\varrho\left(L_{0}\right)$ with eigenvalue $\frac{1}{16}-n<0$ cannot exist, implying $\varrho\left(L_{n}\right)|0\rangle=0$ for $n>0$. This proves 2 for $I=\frac{1}{2}$. Assertion 3 is a corollary of 1 and 2 .

Consider now $I=1$. The decomposition $\pi_{\mathrm{NS}} \cong \pi_{0} \oplus \pi_{1}$ and Lemma 3.4 imply

$$
\pi_{0} \circ \varrho_{1} \oplus \pi_{1} \circ \varrho_{1} \cong \pi_{0} \oplus \pi_{1} .
$$

Since $\pi_{1} \circ \varrho_{1 / 2} \neq 0$, it follows that either $\pi_{0} \circ \varrho_{1}=\pi_{1}$ or $\pi_{0} \circ \varrho_{1} \cong \pi_{0}$. The first possibility will be selected by identifying the lowest weight:

$$
\pi_{0} \circ \varrho_{1}\left(L_{0}\right)|0\rangle=\left(L_{0}+\frac{1}{2}\right)|0\rangle=\frac{1}{2}|0\rangle
$$

since $|0\rangle=|\mathrm{NS}\rangle$ and $b_{1 / 2}|\mathrm{NS}\rangle=0$. By the same argument as for $I=\frac{1}{2}$, it follows that $\pi_{1} \circ \varrho_{1}\left(L_{n}\right)|0\rangle=0$ for $n>0$. This establishes 1 and 2 for $I=1$. Assertion 3 follows again as a corollary.

It is instructive to recompute $\left\langle\lambda_{1 / 2}|T(z)| \lambda_{1 / 2}\right\rangle=z^{-2}\left\langle\lambda_{1 / 2}\left|L_{0}\right| \lambda_{1 / 2}\right\rangle$ starting from the definition of $T(z)$ in Sect. 2.3,

$$
\begin{aligned}
\left\langle\lambda_{1 / 2}|T(z)| \lambda_{1 / 2}\right\rangle= & \left\langle 0\left|\varrho_{1 / 2}(T(z))\right| 0\right\rangle \\
= & -\frac{1}{2} \lim _{w \rightarrow z}\left[\langle 0| \frac{1}{2} \varrho_{1 / 2}\left(\psi(w) \psi^{\prime}(z)\right)\right. \\
& +\frac{1}{2} \varrho_{1 / 2}\left(\psi\left(w e^{2 \pi i}\right) \varrho_{1 / 2}\left(\psi^{\prime}\left(z e^{2 \pi i}\right)|0\rangle-(w-z)^{-2}\right] .\right.
\end{aligned}
$$

Since $\varrho_{1 / 2}$ carries $2 \pi$ periodic field components into antiperiodic ones and vice versa, and only the periodic ones are nonvanishing in the NS-representation, only antiperiodic field components contribute. So

$$
\left\langle\lambda_{1 / 2}\right|\left(T(z)\left|\lambda_{1 / 2}\right\rangle=-\frac{1}{2} \lim _{w \rightarrow z}\left[\frac{\partial}{\partial z}\left\langle 0\left|\varrho_{1 / 2}(\psi(w) \psi(z))\right| 0\right\rangle-(w-z)^{-2}\right] .\right.
$$

The 2-point function is obtained from the same computation as after the proof of Lemma 3.4. Thus

$$
\begin{aligned}
\left\langle\lambda_{1 / 2}|T(z)| \lambda_{1 / 2}\right\rangle & =-\frac{1}{2} \lim _{w \rightarrow z}\left[\frac{1}{2} \frac{\partial}{\partial z}(w-z)^{-1}\left(\sqrt{\frac{z}{w}}+\sqrt{\frac{w}{z}}\right)-(w-z)^{-2}\right] \\
& =\frac{1}{16} z^{-2}
\end{aligned}
$$

in agreement with $\lambda_{1 / 2}=\frac{1}{16}$.

3.3. Interpretation as Lifts of Endomorphisms of an Orthogonal Group. Index. The space $\Gamma_{\mathrm{NS}} \oplus \Gamma_{\mathrm{R}}$ of continuous sections of real line bundles on $\mathbf{S}^{1}$ admits a scalar product

$$
\left\langle f_{1}, f_{2}\right\rangle=\oint_{\widetilde{\mathbf{S}}^{1}} \frac{d z}{4 \pi i z} f_{1}(z) f_{2}(z)
$$

\footnotetext{
${ }^{2}$ We shall often write $L_{n}$ for $\pi_{0}\left(L_{n}\right)$ etc. when acting on states in $\mathscr{H}_{0}$
} 
Completion in the norm $\langle f, f\rangle^{1 / 2}$ yields a real Hilbert space. It consists of those elements of the corresponding complex Hilbert space $\mathbf{H}_{\mathrm{NS}} \oplus \mathbf{H}_{\mathrm{R}}$ which are invariant under complex conjugation $\Theta$,

$$
\Theta f(z)=\bar{f}(z) .
$$

We consider linear maps (= operators)

$$
V: \Gamma_{\mathrm{NS}} \oplus \Gamma_{\mathrm{R}} \rightarrow \Gamma_{\mathrm{NS}} \oplus \Gamma_{\mathrm{R}}
$$

which are either even or odd in the following sense.

Even maps restrict to maps

$$
v: \Gamma_{\mathrm{NS}} \rightarrow \Gamma_{\mathrm{NS}} \text { and } \bar{v}: \Gamma_{\mathrm{R}} \rightarrow \Gamma_{\mathrm{R}} \quad \text { (even) }
$$

while odd maps restrict to maps

$$
v: \Gamma_{\mathrm{NS}} \rightarrow \Gamma_{\mathrm{R}} \text { and } \bar{v}: \Gamma_{\mathrm{R}} \rightarrow \Gamma_{\mathrm{NS}} \quad \text { (odd). }
$$

Even maps $U$ which preserve the scalar products form an orthogonal group. They extend to unitary operators on $\mathbf{H}_{\mathrm{NS}} \oplus \mathbf{H}_{\mathrm{R}}$ which commute with $\Theta$. By definition, operators $U$ have a determinant if $U-1$ is traceclass. The determinant can assume the values +1 and -1 for orthogonal maps.

The group

$$
S O_{e}\left(\mathbf{H}_{\mathrm{NS}} \oplus \mathbf{H}_{\mathrm{R}}\right)
$$

consists of even operators $U: \mathbf{H}_{\mathrm{NS}} \oplus \mathbf{H}_{\mathrm{R}} \rightarrow \mathbf{H}_{\mathrm{NS}} \oplus \mathbf{H}_{\mathrm{R}}$ which preserve the scalar product and have restrictions to $\mathbf{H}_{\mathrm{NS}}$ and $\mathbf{H}_{\mathrm{R}}$ which possess determinant +1 .

Finally we turn to endomorphisms of groups and Lie algebras. Let $V: \Gamma_{\mathrm{NS}} \oplus \Gamma_{\mathrm{R}}$ $\rightarrow \Gamma_{\mathrm{NS}} \oplus \Gamma_{\mathrm{R}}$ be a linear map which is either even or odd and satisfies

$$
V^{*} V=1, \quad \operatorname{dim} \operatorname{ker} V^{*}<\infty
$$

(partial isometry). Given such $V$, an endomorphism $\varrho$ of the group $S O_{e}\left(\mathbf{H}_{\mathrm{NS}} \oplus \mathbf{H}_{\mathrm{R}}\right)$ is obtained which takes

$$
\varrho(U)=\mathbf{1}+V(U-1) V^{*} .
$$

It is easily checked that indeed $\varrho\left(U_{1} U_{2}\right)=\varrho\left(U_{1}\right) \varrho\left(U_{2}\right), \varrho(\mathbf{1})=\mathbf{1}$, and $\varrho(U) \varrho\left(U^{-1}\right)=\mathbf{1}$. Moreover, $\varrho(U)-\mathbf{1}$ is traceclass if $U-\mathbf{1}$ is traceclass.

The universal Majorana algebra Maj consists of smeared fields $\psi(f)$ with $f=f_{\mathrm{NS}}+f_{\mathrm{R}} \in \Gamma_{\mathrm{NS}} \oplus \Gamma_{\mathrm{R}}$ and central elements $1, Y$, with anticommutation relations

$$
\{\psi(f), \psi(g)\}=\frac{1}{2}[1-Y]\left\langle f_{\mathrm{NS}}, g_{\mathrm{NS}}\right\rangle+\frac{1}{2}[1+Y]\left\langle f_{\mathrm{R}}, g_{\mathrm{R}}\right\rangle .
$$

The action of partial isometries $V$ on $\Gamma_{\mathrm{NS}} \oplus \Gamma_{\mathrm{R}}$ lifts to an endomorphism $\varrho$ of Maj

$$
\begin{gathered}
\varrho(\psi(f))=\psi(V f), \\
\varrho(Y)= \pm Y
\end{gathered}
$$

with + if $V$ is even and - if $V$ is odd. If $V$ is odd then $(V f)_{\mathrm{NS}}=V f_{\mathrm{R}}$ and vice versa, while $(V f)_{\alpha}=V f_{\alpha}$ for $\alpha=\mathrm{NS}, \mathrm{R}$ if $V$ is even. It follows then straight from the relation $V^{*} V=1$ that the anticommutation relations are preserved.

The endomorphisms $\varrho$ of the Majorana algebra restrict to endomorphisms of the Lie algebra which is spanned by quadratics $\psi\left(f_{1}\right) \psi\left(f_{2}\right)$ that commute with rotation by $2 \pi$ (i.e. either $f_{1}$ and $f_{2}$ are both in $\Gamma_{\mathrm{NS}}$ or both in $\Gamma_{\mathrm{R}}$ ). If $V$ maps smooth functions into smooth functions then this will give an endomorphism of the global 
Lie algebra Lie $\mathscr{A}$. In favorable circumstances, which prevail in the examples (3.38), (3.39) given below, this extends to an endomorphism of Lie $\bar{A}$.

Let us write $\varrho_{V}$ for the endomorphism induced by $V$. If $V_{1}$ and $V_{2}$ are two partial isometries then $V_{1} V_{2}$ is also a partial isometry and

$$
\varrho_{V_{1}} \varrho_{V_{2}}=\varrho_{V_{1} V_{2}} \text {. }
$$

Endomorphisms $\varrho_{V}$ and equivalence classes of representations $\left[\varrho_{V}\right] \cong \pi_{0} \circ \varrho_{V}$ can be classified by the index of $V$. We define

$$
\text { index }\left[\varrho_{V}\right]=\operatorname{index} \varrho_{V}=\operatorname{index} V \text {. }
$$

Since $V^{*} V=1$ implies $\operatorname{ker} V=0$, it follows that

$$
\text { index } V=\operatorname{dim} \operatorname{ker} V-\operatorname{dim} \operatorname{coker} V \leqq 0 \text {. }
$$

Remembering that coker $V$ is the orthogonal complement of the range of $V$, it follows trivially from $\operatorname{ker} V=0$ that the index is additive

$$
\text { index } V_{1} V_{2}=\text { index } V_{1}+\text { index } V_{2} \text {. }
$$

The explicit examples of endomorphisms $\varrho_{1 / 2}$ and $\varrho_{1}$ of Lie $\overline{\mathscr{A}}$ which were described in the previous subsections, come from partial isometries $V_{1 / 2}$ and $V_{1}$ respectively. Explicitly, their action on basis vectors $e_{a}(z)=z^{a}\left(a \in \frac{1}{2} \mathbf{Z}\right)$ is given by

$$
V_{1 / 2} e_{a}= \begin{cases}i e_{a+1 / 2}, & a>0 \\ \frac{i}{\sqrt{2}}\left(e_{1 / 2}-e_{-1 / 2}\right), & a=0 \\ -i e_{a-1 / 2}, & a<0\end{cases}
$$

and

$$
V_{1} e_{a}= \begin{cases}-e_{a} & a \neq 0, \pm \frac{1}{2} \\ e_{-a} & a=0, \pm \frac{1}{2}\end{cases}
$$

Their index is easily read off

$$
\text { index } V_{1 / 2}=-2, \quad \text { index } V_{1}=0 \text {. }
$$

Let $\mathscr{Z}$ be the center of our global Lie algebra of observables Lie $\overline{\mathscr{A}}$. It is spanned by two elements 1 and $Y$. Lie $\overline{\mathscr{A}}$ is a central extension of the Lie algebra ad Lie $\overline{\mathscr{A}}$ $=\operatorname{Lie} \overline{\mathscr{A}} / \mathscr{Z}$. A unitary action of selfadjoint generators ad $X$ of ad Lie $\overline{\mathscr{A}}$ on $\Gamma_{\mathrm{NS}} \oplus \Gamma_{\mathrm{R}}$ can be defined by

$$
[X, \psi(f)]=\psi(\operatorname{ad} X \cdot f) .
$$

It can be shown that the selfadjoint generators $L_{n}+L_{-n}, i\left(L_{n}-L_{-n}\right), N_{k}+N_{-k}$, $i\left(N_{k}-N_{-k}\right), J_{a b}+J_{-a-b}$, and $i\left(J_{a b}-J_{-a-b}\right)$ in ad Lie $\overline{\mathscr{A}}$ are generators of 1-parameter groups of orthogonal transformations of $\mathbf{H}_{\mathrm{NS}} \oplus \mathbf{H}_{\mathrm{R}}$. This is seen as follows. We introduce a complex basis for $\mathbf{H}=\mathbf{H}_{\mathrm{NS}} \oplus \mathbf{H}_{\mathrm{R}}$. It consists of smooth sections $e_{a} \in \mathbf{H}_{\mathrm{NS}}, a \in \mathbf{Z}+\frac{1}{2}$, and $e_{a} \in \mathbf{H}_{\mathrm{R}}, a \in \mathbf{Z}$, given by

$$
e_{a}(z)=z^{a}
$$

$( \pm 1)^{1 / 2}\left(J_{a b} \pm J_{-a-b}\right)$ generate rotations of $e_{ \pm a}$ into $e_{ \pm b}$, leaving $e_{c}$ fixed for $c \neq \pm a, \pm b$. In particular $J_{a,-a}$ rotates $e_{ \pm a} \rightarrow e^{ \pm i \phi} e_{ \pm a}( \pm 1)^{1 / 2}\left(L_{n} \pm L_{-n}\right)$ generate 1 -parameter groups of diffeomorphisms. Their unitary action on $\mathbf{H}$ is given by the 
well known imbedding [6] $\operatorname{Diff}\left(\mathbf{S}^{1}\right) \subset U_{\text {res }}(\mathbf{H})$. This restricts to an orthogonal action on $\Gamma_{\mathrm{NS}} \oplus \Gamma_{\mathrm{R}}$ (the definition of $U_{\text {res }}$ involves the natural polarization $\mathbf{H}=\mathbf{H}_{+} \oplus \mathbf{H}_{-}$, where $\mathbf{H}_{+}$is spanned by $e_{a}$ with $a>0$ ).

Finally, the remaining generators $( \pm 1)^{1 / 2}\left(N_{k} \pm N_{-k}\right)$ need to be examined. One shows that $\exp \left[-t\left(N_{k}+N_{-k}\right)\right] e_{a}$ admits a convergent power series expansion in $t$ for small enough $t$, and similarly for $i\left(N_{k}-N_{-k}\right)$.

3.4. Localized Endomorphisms. We retain the convention that $I \subset \mathbf{S}^{1}$ is called an (open) interval if it is (open and) connected, and its closure is not all of $\mathbf{S}^{1}$.

Given a global (Lie) algebra $\mathscr{A}$ and a net of subalgebras $\mathscr{A}(I)$ associated with intervals $I \subset \mathbf{S}^{1}$, an endomorphism $\varrho$ of $\mathscr{A}$ is said to be localized on $I$ if

$$
\varrho(A)=A \quad \text { whenever } \quad A \in \mathscr{A}\left(I^{\prime}\right), \quad I^{\prime} \cap I=\emptyset .
$$

In the last subsection we saw how endomorphisms $\varrho$ can be obtained from partial isometries $V$ of $\Gamma_{\mathrm{NS}} \oplus \Gamma_{\mathrm{R}}$. Now we study their localization properties.

Fix an interval $I C \mathbf{S}^{1}$. Its complement $I^{\prime}$ on $\mathbf{S}^{1}$ is projection of two disjoint intervals $\widetilde{I}_{+}^{\prime}$ and $\widetilde{I}_{-}^{\prime}$ in the double cover $\widetilde{\mathbf{S}}^{1}$ of $\mathbf{S}^{1}$. Given $I$, consider partial isometries $V: \mathbf{H}_{\mathrm{NS}} \oplus \mathbf{H}_{\mathrm{R}} \rightarrow \mathbf{H}_{\mathrm{NS}} \oplus \mathbf{H}_{\mathrm{R}}$ with the property that

$$
\begin{gathered}
V f(z)=\sigma_{ \pm} f(z) \text { for } z \in \widetilde{I}_{ \pm}^{\prime}, \\
\sigma_{+}^{2}=1=\sigma_{-}^{2} .
\end{gathered}
$$

They will be said to be pseudolocalized on $I$.

Consider now the action of an endomorphism $\varrho$ of Maj which is induced by a partial isometry $V$ that is pseudolocalized in $I$. Remember that $f \in \Gamma_{\mathrm{NS}} \oplus \Gamma_{\mathrm{R}}$ may be regarded as ordinary functions in $\widetilde{\mathbf{S}}^{1}$. Suppose that $f$ has either support on $\widetilde{I}_{+}^{\prime}$ or support on $\tilde{I}_{-}^{\prime}$. Then

$$
\varrho(\psi(f))=\psi(V f)=\sigma_{\alpha} \psi(f) \quad \text { if } \quad \operatorname{supp} f \subset \tilde{I}_{\alpha}^{\prime}, \quad \alpha= \pm .
$$

For odd maps $\sigma_{+} \sigma_{-}=-1$. However, the sign factors cancel when we consider observables. Consider for instance a quadratic $\psi\left(f_{1}\right) \psi\left(f_{2}\right) \in \operatorname{Lie} \mathscr{A}\left(I_{1}\right)$ with $I_{1} \cap I=\emptyset$. Lie $\mathscr{A}(I)$ was originally made out of NS-Majorana fields (smeared with test functions $f \in \Gamma_{\mathrm{NS}}$ with support in $I_{1}$ ) and then injected into Lie $\mathscr{A}$,

$$
\psi\left(f_{1}\right) \psi\left(f_{2}\right) \rightarrow \frac{1}{2} \psi\left(f_{1}^{+}\right) \psi\left(f_{2}^{+}\right)+\frac{1}{2} \psi\left(f_{1}^{-}\right) \psi\left(f_{2}^{-}\right),
$$

where $f_{1}^{+}$and $f_{2}^{-}$are functions on $\tilde{\mathbf{S}}^{1}$ with support in $\tilde{I}_{1}^{+}$and $\tilde{I}_{2}^{-}$respectively (they are determined up to a common sign by the values of $f$ in the local chart $I$ ). It follows from Eq. (3.45) that

$$
\varrho(A)=A
$$

for $A=\frac{1}{2} \psi\left(f_{1}^{+}\right) \psi\left(f_{2}^{+}\right)+\frac{1}{2} \psi\left(f_{1}^{-}\right) \psi\left(f_{2}^{-}\right)$with supp $f_{i}^{ \pm} \in \tilde{I}_{ \pm}^{\prime}$. The result generalizes to all elements of Lie $\mathscr{A}(I)$ because they may be written as infinite sums of quadratics as we have just considered. It generalizes also to endomorphisms $\varrho$ of Lie $\overline{\mathscr{A}}$, with local algebras $\operatorname{Lie} \mathscr{A}(I) \oplus \operatorname{Vir}(I)$ injected, by a similar argument. In conclusion we have obtained

Proposition 3.6. If the endomorphism $\varrho$ of the Lie algebra of observables Lie $\mathscr{A}$ or Lie $\bar{A}$ is induced by partial isometry $V$ of $\Gamma_{\mathrm{NS}} \oplus \Gamma_{\mathrm{R}}$ which is pseudolocalized in I, then $\varrho$ is localized in I. 
We will now present examples of endomorphisms of index -2 which are localized in domains $I_{k}$ of length $\frac{2 \pi}{k}$ in angle, $k=2,3, \ldots$. Conjugation with rotations of the circle [or with $S L(2, \mathbf{C})$ transformations] will yield endomorphisms with arbitrary domains of localization. In this way one obtains sequences of endomorphisms $\varrho_{k}$ which are odd in the sense that $\varrho_{k}(Y)=-Y$, and whose support shrink to an arbitrary point $\{z\}$ as $k \rightarrow \infty$.

Let $\zeta=-1$ and consider intervals

$$
\begin{gathered}
I_{k}=\left\{z=e^{i \phi},-\frac{\pi}{k} \leqq \phi \leqq \frac{\pi}{k}\right\}, \\
\mathbf{S}^{1} \backslash\{\zeta\}=I_{k}+I_{-}+I_{+},
\end{gathered}
$$

with $-\pi<\phi<-\frac{\pi}{k}$ on $I_{-}$and $\frac{\pi}{k}>\phi>\pi$ on $I_{+}$. Set

$$
e_{a}^{(k)}(z)=\sqrt{k} e_{k a}(z) \quad\left(a \in \frac{1}{2} \mathbf{Z}\right)
$$

Functions $e_{r}^{(k)}$ with $r \in \mathbf{Z}+\frac{1}{2}$ form an orthogonal basis in the space $L^{2}\left(I_{k}, d \phi / 2 \pi\right)$ with scalar product $\langle,\rangle_{k}$. Thus

$$
e_{a}(z)=\sum_{s \in \mathbf{Z}+1 / 2}\left\langle e_{s}^{(k)}, e_{a}\right\rangle_{k} e_{s}^{(k)}(z) \text { for } z \in I_{k}
$$

Define

$$
f_{r}^{(k)}(z)= \begin{cases}i e_{r+1 / 2}^{(k)}(z), & r=\frac{1}{2}, \frac{3}{2}, \ldots \\ -i e_{r-1 / 2}^{(k)}(z), & r=-\frac{1}{2},-\frac{3}{2}, \ldots\end{cases}
$$

It follows that

b)

$$
\begin{gathered}
\left\langle f_{r}^{(k)}, f_{s}^{(k)}\right\rangle_{k}=\left\langle e_{r}^{(k)}, e_{s}^{(k)}\right\rangle_{k}=\delta_{r, s}, \\
f_{r}^{(k)}\left(z_{ \pm}\right)=\mp e_{r}^{(k)}\left(z_{ \pm}\right) \text {for } \quad z_{ \pm}=e^{ \pm i \pi / k} .
\end{gathered}
$$

The partial isometry $V$ is defined by

$$
\begin{gathered}
V e_{a}(z)=\sum_{r \in \mathbf{Z}+1 / 2}\left\langle e_{r}^{(k)}, e_{a}\right\rangle f_{r}^{(k)}(z) \text { for } z \in I_{k}, \\
V e_{a}(z)=\mp e_{a}(z) \text { for } z \in I_{ \pm} \quad\left(a \in \frac{1}{2} \mathbf{Z}\right) .
\end{gathered}
$$

It commutes with complex conjugation $\Theta$. To verify that $V$ is a partial isometry it suffices to show that

$$
\left\langle V e_{a}, V e_{c}\right\rangle_{k}=\left\langle e_{a}, e_{c}\right\rangle_{k} .
$$

Validity of this equation follows from definition (3.53) by inserting Eqs. (3.52), (3.50). $V$ is pseudolocalized on $I_{k}$ by definition (3.54). One has $\operatorname{tr}\left(1-V V^{*}\right)=1$ both on $\mathbf{H}_{\mathrm{NS}}$ and on $\mathbf{H}_{\mathrm{R}}$. As a result

$$
U \rightarrow \varrho(U)=1-V(U-1) V^{*}
$$

is a well defined endomorphism of $S O_{e}\left(\mathbf{H}_{\mathrm{NS}} \oplus \mathbf{H}_{\mathrm{R}}\right)$ which is localized on $I_{k}$; and index $\varrho \equiv$ index $V=-2$.

Reflections are examples of even partial isometries. Let $g=g_{\mathrm{NS}} \oplus g_{\mathrm{R}} \in \Gamma_{\mathrm{NS}} \oplus \Gamma_{\mathrm{R}}$ with support, as a function on $\widetilde{\mathbf{S}}^{1}$, which projects to $I \subset \mathbf{S}^{1}$, and normalization 
$\left\langle g_{N S}, g_{N S}\right\rangle=2=\left\langle g_{R}, g_{R}\right\rangle$. Then the reflection

$$
v: f \rightarrow-f+\langle f, g\rangle g
$$

is an even partial isometry and pseudolocalized in $I$. It induces an automorphism which is inner on $\mathrm{Maj}_{\mathrm{NS}}$ and $\mathrm{Maj}_{\mathrm{R}}$, but outer on $S O_{e}\left(\mathbf{H}_{\mathrm{NS}} \oplus \mathbf{H}_{\mathrm{R}}\right)$. Our (nonpseudolocalized) standard $V_{1}$ is also of the form (3.56) with $g=\sqrt{2} e_{0}+e_{1 / 2}+e_{-1 / 2}$.

\section{Fusion Rules and Intertwining Operators Between Representations}

4.1. Fusion Rules. We retain the notation [@] for the equivalence class of the unitary representation $\pi_{0} \circ \varrho$ of the Lie algebra of observables Lie $\overline{\mathscr{A}} \supset$ Vir. Remember that $\pi_{0}$ is the vacuum representation of Lie $\overline{\mathscr{A}}$. It was obtained by extending the positive energy representation of Vir with lowest weight 0 to a representation of Lie $\overline{\mathscr{A}}$ which acts in the same Hilbert space $\mathscr{H}_{0}$.

We found a complete set of representative endomorphisms $\varrho_{J}$ of Lie $\bar{A}$, $J=0, \frac{1}{2}, 1$, such that $\left[\varrho_{J}\right]$ are all the positive energy representations of Vir, extended to $\operatorname{Lie} \bar{A}$.

The product of two endomorphisms is also an endomorphism. Therefore $\pi_{0} \circ \varrho_{K} \varrho_{J}$ is also a unitary representation of Lie $\overline{\mathscr{A}}$. It restricts to a positive energy representation of Vir. [The positive energy property holds because $L_{0}$ is a positive element of Maj (i.e. a sum of terms $\psi \psi^{*}$ ), $\varrho_{J}$ extend to Maj, and $A \geqq 0$ implies $\varrho(A) \geqq 0$ by the *-endomorphism property.]

The representation $\pi_{0}{ }^{\circ} \varrho_{K} \varrho_{J}$ may be reducible. We will find its decomposition into irreducibles in the form

$$
\left[\varrho_{L} \varrho_{J}\right]=\sum_{K} N_{J K}^{(L)}\left[\varrho_{K}\right]
$$

This is the decomposition into irreducible representations of Lie $\bar{A}$ and at the same time the decomposition into irreducibles for Vir.

We will write $A$ for $\pi_{0}(A)$ when it is clear that it acts in $\mathscr{H}_{0}$. All the representations $\pi_{0} \circ \varrho, \varrho=\varrho_{J}, \varrho_{J} \varrho_{K}, \ldots$ act in the same Hilbert space $\mathscr{H}_{0}$. The projection operators $\Pi_{K}^{(I, J)}$ in $\mathscr{H}_{0}$ which project on the irreducible subrepresentations, must commute with the representation operators. Thus

$$
\Pi_{K}^{(I, J)} \in \varrho_{1 / 2}^{2}(\text { Lie } \overline{\mathscr{A}})^{\prime}
$$

[commutant in $\left.\mathscr{B}\left(\mathscr{H}_{0}\right)\right]$. We find these projection operators in the even part of $\mathrm{Maj}_{\mathrm{NS}}$, which is the same as the universal enveloping algebra of the complex Lie algebra $\pi_{0}(\operatorname{Lie} \mathscr{A})$. In fact they happen to be in Lie $\mathscr{A}$.

Consider first $\left[\varrho_{1 / 2} \varrho_{1 / 2}\right]$. From the definition of $\varrho_{1 / 2}$ one finds

$$
\varrho_{1 / 2}^{2}\left(b_{r}\right)=\left\{\begin{array}{ll}
-b_{r+1}, & r>0 \\
-b_{r-1}, & r<0
\end{array} \text { for } r \in \mathbf{Z}+\frac{1}{2}\right.
$$

while $\varrho_{1 / 2}^{2}\left(b_{n}\right)=0$ in the vacuum representation $\pi_{0}$, for $n \in \mathbf{Z}$. It follows from the canonical anticommutation relations in $\mathrm{Maj}_{\mathrm{NS}}$ that

$$
\Pi_{0}=b_{1 / 2} b_{-1 / 2}, \quad \Pi_{1}=1-\Pi_{0}
$$

are two orthogonal projection operators in the commutant of $\varrho_{1 / 2}^{2}(\mathrm{Lie} \overline{\mathscr{A}})$. We show that $\Pi_{0} \mathscr{H}_{0}$ and $\Pi_{1} \mathscr{H}_{0}$ carry representations in the equivalence class of $\pi_{0}$ and $\pi_{0} \circ \varrho_{1}$, respectively. 
To do this, we exhibit bijective maps

$$
R^{*}: \Pi_{0} \mathscr{H}_{0} \rightarrow \mathscr{H}_{0}, \quad S^{*}: \Pi_{1} \mathscr{H}_{0} \rightarrow \mathscr{H}_{0}
$$

such that

$$
\begin{gathered}
A R^{*}=R^{*} \varrho_{1 / 2}^{2}(A), \\
\varrho_{1}(A) S^{*}=S^{*} \varrho_{1 / 2}^{2}(A) .
\end{gathered}
$$

It follows from the definitions that $\varrho_{1}^{2}=i d$ and $\varrho_{1} \varrho_{1 / 2}=\varrho_{1 / 2}$ (see below). Applying $\varrho_{1}$ to Eq. $(4.5 b)$, we see that its solution is

$$
S^{*}=\varrho_{1}\left(R^{*}\right),
$$

with $R^{*}$ a solution of the first equation. The solution for $R^{*}$ is unique, apart from an overall phase factor. If $R^{\prime *}$ were another one, $R^{*} R^{\prime}: \mathscr{H}_{0} \rightarrow \mathscr{H}_{0}$ would have to commute with the action of Lie $\overline{\mathscr{A}}$ in $\mathscr{H}_{0}$, implying $R^{*} R^{\prime} \in \mathbf{C} \cdot \mathbf{1}$ by irreducibility.

A basis for $\mathscr{H}_{0}$ is formed by vectors

$$
\left|r_{2 N}, \ldots, r_{1}\right\rangle=b_{-r_{2 N}} \ldots b_{-r_{1}}|0\rangle, \quad 0<r_{1}<\ldots<r_{2 N}, \quad r_{i} \in \mathbf{Z}+\frac{1}{2}, \quad N=0,1, \ldots
$$

We extend $R^{*}$ to all of $\mathscr{H}_{0}$ by putting $R^{*} \Pi_{1} \mathscr{H}_{0}=0 . R^{*}$ is described explicitly by its action on basis vectors

$$
R^{*}\left|r_{2 N} \ldots r_{1}\right\rangle=\left\{\begin{array}{lll}
\left|r_{2 N}-1 \ldots r_{1}-1\right\rangle & \text { if } r_{1} \geqq \frac{3}{2} \\
0 & \text { if } & r_{1}=\frac{1}{2}
\end{array} .\right.
$$

For $N=0$ this is to be read as

$$
R^{*}|0\rangle=|0\rangle
$$

As a result

$$
R R^{*}=\Pi_{0} \quad \text { and } \quad R^{*} R=\mathbf{1} .
$$

Since $\varrho_{1}\left(\Pi_{0}\right)=\Pi_{1}$ it follows that

$$
S S^{*}=\Pi_{1} \quad \text { and } \quad S^{*} S=\mathbf{1} .
$$

This shows that the maps (4.4) are bijective. One verifies by explicit computation that the intertwining relations (4.5) are fulfilled for all $A=b_{a} b_{c}$, and more generally for all generators of Lie $\overline{\mathscr{A}}$ (on their common dense invariant domain of definition, which consists of finite sums of basis vectors).

$R^{*}$ can be written as an infinite product

$$
\begin{aligned}
& R^{*}=\ldots A_{3} A_{2} A_{1} \Pi_{0}=\left(\prod_{n \geqq 1} A_{n}\right) \Pi_{0}, \\
& A_{n}=b_{n+1 / 2} b_{-n-1 / 2}+b_{-n+1 / 2} b_{n+1 / 2} .
\end{aligned}
$$

Factors $A_{n}$ with larger $n$ stand further to the left. On finite sums of basis vectors, all but a finite number of factors $A_{n}$ act like the identity operator. Since $R^{*}$ is a partial isometry, its definition extends to all of $\mathscr{H}_{0} . S^{*}=\varrho_{1}\left(R^{*}\right)$ admits a similar infinite product representation. This completes the proof that $\left[\varrho_{1 / 2} \varrho_{1 / 2}\right]=\left[\varrho_{0}=i d\right]+\left[\varrho_{1}\right]$.

Next we consider $\left[\varrho_{1}^{2}\right]$. From the definition of $\varrho_{1}$ one finds

$$
\varrho_{1}^{2}=i d \text {. }
$$

Therefore $\left[\varrho_{1}^{2}\right]=\left[\varrho_{0}=i d\right]$. 
Similarly one finds $\varrho_{1} \varrho_{1 / 2}\left(b_{a}\right)=-\varrho_{1 / 2}\left(b_{a}\right)$ for $a \in \mathbf{Z}$ while both sides vanish in the vacuum representation for $a \in \mathbf{Z}+\frac{1}{2}$, and $\varrho_{1} \varrho_{1 / 2}(Y)=\varrho_{1 / 2}(Y)=-Y$. Therefore

$$
\varrho_{1} \varrho_{1 / 2}=\varrho_{1 / 2}
$$

on Lie $\overline{\mathscr{A}}$. This implies $\left[\varrho_{1} \varrho_{1 / 2}\right]=\left[\varrho_{1 / 2}\right]$.

Finally we should determine $\left[\varrho_{1 / 2} \varrho_{1}\right]$. The general theory says that $[3]$

$$
\left[\varrho_{K} \varrho_{J}\right]=\left[\varrho_{J} \varrho_{K}\right]
$$

under standard assumptions. In our model, the only case which needs to be checked is $\left[\varrho_{1 / 2} \varrho_{1}\right]=\left[\varrho_{1} \varrho_{1 / 2}\right]$. We have

$$
\varrho_{1 / 2} \varrho_{1}\left(b_{n}\right)= \begin{cases}-i b_{n+1 / 2}, & n>0 \\ \frac{i}{\sqrt{2}}\left(b_{1 / 2}-b_{-1 / 2}\right), & n=0 \\ i b_{n-1 / 2}, & n<0\end{cases}
$$

and $\varrho_{1 / 2} \varrho_{1}\left(b_{r}\right)=0$ in the vacuum representation for $r \in \mathbf{Z}+\frac{1}{2}$, while $\varrho_{1 / 2} \varrho_{1}(Y)=-Y$ $=\varrho_{1 / 2}(Y)$. We exhibit a unitary operator

such that

$$
U^{*}: \mathscr{H}_{0} \rightarrow \mathscr{H}_{0}
$$

$$
\varrho_{1} \varrho_{1 / 2}(A) U^{*}=U^{*} \varrho_{1 / 2} \varrho_{1}(A) \text { on } \mathscr{H}_{0}
$$

for all bilinears $A=b_{a} b_{c}$ in the Majorana fields, and more generally for all elements $A \in \operatorname{Lie} \overline{\mathscr{A}}$. This will show that $\pi_{0} \circ \varrho_{1 / 2} \varrho_{1}$ and $\pi_{0} \circ \varrho_{1 / 2}$ are indeed unitarily equivalent. Therefore $\left[\varrho_{1 / 2} \varrho_{1}\right]=\left[\varrho_{1 / 2}\right]=\left[\varrho_{1} \varrho_{1 / 2}\right]$ as expected. Explicitly, $U^{*}$ is given by $U^{*}=1-2 b_{-1 / 2} b_{1 / 2}$.

In conclusion we have proven the fusion rules, Theorem 4 of the introduction. We note that these fusion rules agree with the known fusion rules for the minimal model with central charge $c=\frac{1}{2}$.

4.2. Intertwiners in the Algebra. Let us recall the defining property of an intertwiner $T$ between endomorphisms $\varrho_{1}$ and $\varrho_{2}$. We say that

$$
T \in \operatorname{Hom}\left(\varrho_{1}, \varrho_{2}\right) \text { if } T \varrho_{1}(A)=\varrho_{2}(A) T \text { for all } A \text {. }
$$

We are studying endomorphisms of a Lie algebra Lie $\mathscr{A}$, which possess (unique) extensions to Lie $\overline{\mathscr{A}} \supset$ Lie $\mathscr{A}$. In this context, intertwiners $T$ are sought in the universal enveloping algebra $U(\operatorname{Lie} \mathscr{A})$ with relations imposed which come from

$$
Y b_{a}=(-1)^{2 a} b_{a} \text {. }
$$

It will however be necessary to include in this algebra also some infinite products which are well defined in all positive energy representations of Lie $\mathscr{A}$

$$
A \in \operatorname{Lie} \mathscr{A}, \quad T \in U(\operatorname{Lie} \mathscr{A}) \text {. }
$$

If $\pi$ is any positive energy representation of Lie $\mathscr{A}$ [and therefore of $U($ Lie $\mathscr{A})]$ then $\pi(T)$ is an intertwining operator between representations $\pi \circ \varrho_{1}$ and $\pi \circ \varrho_{2}$,

$$
\pi(T) \pi_{1}(A)=\pi_{2}(A) \pi(T) \text { for } \pi_{i}=\pi \circ \varrho_{i} \text {, if } T \in \operatorname{Hom}\left(\varrho_{1}, \varrho_{2}\right) .
$$

The existence of a nonvanishing intertwining operator $\pi(T)$ between representations $\pi_{1}$ and $\pi_{2}$ means that these representations have a common subrepresen- 
tation. It follows by taking adjoints that

$$
\pi_{1}(A) \pi\left(T^{*}\right)=\pi\left(T^{*}\right) \pi_{2}(A) \text { for all } A .
$$

Therefore $\pi\left(T^{*} T\right)$ commutes with $\pi_{1}(A)$ for all $A$, and $\pi\left(T T^{*}\right)$ commutes with $\pi_{2}(A)$ for all $A$. If $\pi_{1}$ is irreducible, this implies that $\pi\left(T^{*} T\right) \in \mathbf{C} \cdot \mathbf{1}$. By suitable normalisation of $T$ one obtains

$$
\pi\left(T^{*} T\right)=1, \quad \pi\left(T T^{*}\right)=\Pi_{1} .
$$

$\Pi_{1}=$ projection operator on the subrepresentation of $\pi_{2}$ which is equivalent to $\pi_{1}$. We are interested in the intertwiner $T\left(\begin{array}{cc}I \\ K & J\end{array}\right)$ between $\varrho_{J} \varrho_{I}$ and $\varrho_{K}$.

$$
T=T\left(\begin{array}{cc}
I \\
K & J
\end{array}\right) \in \operatorname{Hom}\left(\varrho_{J} \varrho_{I}, \varrho_{K}\right) \text {. }
$$

They obey

$$
\varrho_{K}(A) T=T \varrho_{J} \varrho_{I}(A) .
$$

There may be several such intertwiners, if so we distinguish them by a label $a$.

These intertwiners effect the reduction of representation $\left[\varrho_{J} \varrho_{I}\right]$ into irreducibles, as we saw in the last subsection. They will also be basic building blocks of chiral vertex operators.

Our Lie algebra Lie $\mathscr{A}$ has center $\mathscr{Z}$ which is generated by 1 and $Y$. We imposed the relation $Y^{2}=1$ in $U(\operatorname{Lie} \mathscr{A})$. Therefore $\mathscr{Z}$ contains projection operators $\frac{1}{2}[1+Y]$. If $T$ is an intertwining operator, then so are $\frac{1}{2}[1 \pm Y] T$. There are therefore two classes of intertwiners:

$$
\begin{aligned}
\text { NS-intertwiners: } T=\frac{1}{2}[1-Y] T, & T T^{*}=\frac{1}{2}[1-Y], \\
\text { R-intertwiners: } T=\frac{1}{2}[1+Y] T, & T T^{*}=\frac{1}{2}[1+Y] .
\end{aligned}
$$

It follows that there are also two classes of projection operators

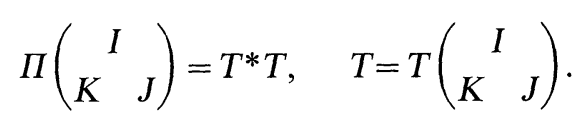

It turns out that in our model there is at most one NS-intertwiner and one R-intertwiner for each triple $\left(\begin{array}{cc}I \\ K & J\end{array}\right)$.

We say that a set of NS-intertwiners (R-intertwiners) is complete if

$$
\sum_{K} \Pi\left(\begin{array}{cc}
I \\
K & J
\end{array}\right)=\frac{1}{2}[1 \mp Y] \quad(- \text { for } \mathrm{NS},+ \text { for } \mathrm{R}) .
$$

Only NS-intertwiners and projectors are nonvanishing in the vacuum representation $\pi_{0}$ since $\pi_{0}(Y)=-1$. They effect the decomposition of the representation $\pi_{0} \circ \varrho_{J} \varrho_{I}$ into irreducibles $\pi_{0} \circ \varrho_{K}$, as we found in the last subsection. The case $I=0$ or $J=0$ is trivial. The other nonvanishing intertwiners and projectors are 
NS-Projectors and Intertwiners (overall factors $\frac{1}{2}[1-Y]$ are understood).

$$
\begin{aligned}
I=\frac{1}{2}: \Pi\left(\begin{array}{cc}
\frac{1}{2} \\
0 & \frac{1}{2}
\end{array}\right) & =b_{1 / 2} b_{-1 / 2}, \quad T\left(\begin{array}{cc}
\frac{1}{2} \\
0 & \frac{1}{2}
\end{array}\right) \equiv R_{\mathrm{NS}}^{*}=\lim _{M \rightarrow \infty} A_{M} \ldots A_{2} A_{1} b_{1 / 2} b_{-1 / 2}, \\
\Pi\left(\begin{array}{cc}
\frac{1}{2} \\
1 & \frac{1}{2}
\end{array}\right) & =b_{-1 / 2} b_{1 / 2}, \quad T\left(\begin{array}{cc}
\frac{1}{2} \\
1 & \frac{1}{2}
\end{array}\right)=\varrho_{1}\left(T\left(\begin{array}{cc}
\frac{1}{2} \\
0 & \frac{1}{2}
\end{array}\right)\right), \\
\Pi\left(\begin{array}{cc}
\frac{1}{2} \\
\frac{1}{2} & 1
\end{array}\right) & =1, \quad T\left(\begin{array}{cc}
\frac{1}{2} \\
\frac{1}{2} & 1
\end{array}\right)=1 \\
I=1: \Pi\left(\begin{array}{cc}
1 \\
\frac{1}{2} & \frac{1}{2}
\end{array}\right) & =1, \quad T\left(\begin{array}{cc}
1 \\
\frac{1}{2} & \frac{1}{2}
\end{array}\right)=1-2 b_{-1 / 2} b_{1 / 2}, \\
\Pi\left(\begin{array}{cc}
1 & \\
0 & 1
\end{array}\right) & =1, \quad T\left(\begin{array}{cc}
1 \\
0 & 1
\end{array}\right)=1,
\end{aligned}
$$

(with $A_{n}=b_{n+1 / 2} b_{-n-1 / 2}+b_{-n+1 / 2} b_{n+1 / 2}$ ).

The R-intertwiners and projectors can be found for instance by looking at the representation $\pi_{1 / 2} \cong \pi_{0}{ }^{\circ} \varrho_{1 / 2}$ in place of $\pi_{0}$. (The representation $\pi_{0} \oplus \pi_{1 / 2}$ is faithful.)

It turns out that nonvanishing R-intertwiners $T\left(\begin{array}{cc}I \\ K & J\end{array}\right)$ exist for exactly the same triples $\left(\begin{array}{cc}I & \\ K & J\end{array}\right)$ as in the case of the NS-intertwiners.

R-Projectors and Intertwiners (overall factors $\frac{1}{2}[1+Y]$ are understood).

$$
\begin{aligned}
& I=\frac{1}{2}: \Pi\left(\begin{array}{cc}
\frac{1}{2} \\
0 & \frac{1}{2}
\end{array}\right)=\frac{1}{2}\left(1+\sqrt{2} b_{0}\left[b_{1}-b_{-1}\right]\right), \\
& T\left(\begin{array}{cc}
\frac{1}{2} \\
0 & \frac{1}{2}
\end{array}\right)=\lim A_{M+1 / 2} \ldots A_{3 / 2} \frac{1}{\sqrt{2}} b_{1}\left(b_{-1}-\sqrt{2} b_{0}\right), \\
& \Pi\left(\begin{array}{cc}
\frac{1}{2} \\
1 & \frac{1}{2}
\end{array}\right)=\frac{1}{2}\left(1-\sqrt{2} b_{0}\left[b_{1}-b_{-1}\right]\right), \quad T\left(\begin{array}{cc}
\frac{1}{2} \\
1 & \frac{1}{2}
\end{array}\right)=\varrho_{1}\left(T\left(\begin{array}{cc}
\frac{1}{2} \\
0 & \frac{1}{2}
\end{array}\right)\right), \\
& \Pi\left(\begin{array}{cc}
\frac{1}{2} \\
\frac{1}{2} & 1
\end{array}\right)=1, \quad T\left(\begin{array}{cc}
\frac{1}{2} \\
\frac{1}{2} & 1
\end{array}\right)=1,
\end{aligned}
$$

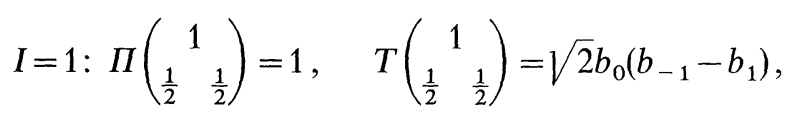

$$
\begin{aligned}
& \Pi\left(\begin{array}{cc}
1 & \\
0 & 1
\end{array}\right)=1, \quad T\left(\begin{array}{cc}
1 \\
0 & 1
\end{array}\right)=1
\end{aligned}
$$

with $A_{n}$ as before.

The limit is understood in the same algebraic sense as before: on finite sums of common eigenvectors of $L_{0}$ and $N_{0}$, the result of the action of

$$
A_{M+1 / 2} \ldots A_{3 / 2} \frac{1}{\sqrt{2}} b_{1}\left(b_{-1}-\sqrt{2} b_{0}\right)
$$

is independent of $M$ for large enough $M$. 
4.3. Intertwining Operators between Superselection Sectors. From the NSintertwining operators in the algebra $U($ Lie $\mathscr{A})$ we may construct intertwining operators between superselection sectors, i.e. between irreducible representation spaces for the observable algebra,

$$
\Gamma\left(\begin{array}{cc}
I \\
K & J
\end{array}\right): \mathscr{H}_{J} \rightarrow \mathscr{H}_{K} .
$$

They are to satisfy the intertwining property

$$
\pi_{K}(A) \Gamma\left(\begin{array}{cc}
I & \\
K & J
\end{array}\right)=\Gamma\left(\begin{array}{cc}
I \\
K & J
\end{array}\right) \pi_{J}\left(\varrho_{I}(A)\right) .
$$

The representation $\pi_{K}$ on $\mathscr{H}_{K}$ is equivalent to $\pi_{0} \circ \varrho_{K}$ on $\mathscr{H}_{0}$ as we know. Let $i_{K}$ be the identification map

$$
i_{K}: \mathscr{H}_{K} \rightarrow \mathscr{H}_{0}, \quad \pi_{0} \circ \varrho_{K}(A) i_{K}=i_{K} \pi_{K}(A) .
$$

If $T\left(\begin{array}{cc}I \\ K & J\end{array}\right)$ is an intertwining operator in the algebra, then

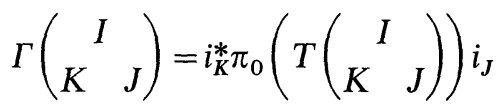

satisfies the intertwining relation. This shows that NS-intertwining operators yield intertwining operators between superselection sectors. A nonvanishing intertwiner will exist if and only if $\left[\varrho_{J} \varrho_{I}\right]$ contains $\left[\varrho_{K}\right]$ as a subrepresentation. In our model there is at most one such intertwining operator.

We turn now to the consideration of field operators which are defined on the total Hilbert space which is the sum of superselection sectors, without multiplicities.

$$
\mathscr{H}=\mathscr{H}_{0} \oplus \mathscr{H}_{1 / 2} \oplus \mathscr{H}_{1} .
$$

This space carries a reducible representation $\pi$ of Lie $\overline{\mathscr{A}}$ given by

$$
\pi(A)|\psi\rangle=\pi_{J}(A)|\psi\rangle \text { for }|\psi\rangle \in \mathscr{H}_{J} .
$$

We construct operators $\Gamma^{I}$ on $\mathscr{H}$ such that

$$
\pi(A) \Gamma^{I}=\Gamma^{I} \pi\left(\varrho_{I}(A)\right) .
$$

Projection operators $E_{J}$ on $\mathscr{H}_{J}$ commute with all $\pi(A)$. Therefore

$$
\Gamma\left(\begin{array}{cc}
I & \\
K & J
\end{array}\right)=E_{K} \Gamma^{I} E_{J}: \mathscr{H}_{J} \rightarrow \mathscr{H}_{\mathrm{K}}
$$

will be an intertwining operator as were considered above. Thus, $\Gamma^{I}$ will have to be put together from such intertwiners.

Consider the action of $\Gamma^{I}$ on $\mathscr{H}_{J}$ for fixed $I, J$. The crucial observation is that $\mathscr{H}_{J}$ is the orthogonal direct sum of the support of $\Gamma\left(\begin{array}{cc}I \\ K & J\end{array}\right)$

$$
\mathscr{H}_{J}=\bigoplus_{\boldsymbol{K}} \operatorname{supp} \Gamma\left(\begin{array}{cc}
I \\
K & J
\end{array}\right) \text {. }
$$


This follows from the fact that the $T\left(\begin{array}{cc}I \\ K & J\end{array}\right)$ form a complete system of NSintertwining operators. From this we obtain

Proposition 4.2. Define the operator $\Gamma^{I}$ on $\mathscr{H}=\oplus \mathscr{H}_{J}$ by

$$
\Gamma^{I}=\Gamma\left(\begin{array}{cc}
I & \\
K & J
\end{array}\right) \text { on } \operatorname{supp} \Gamma\left(\begin{array}{cc}
I \\
K & J
\end{array}\right) \subset \mathscr{H}_{J}
$$

Then

$$
\pi(A) \Gamma^{I}=\Gamma^{I} \pi\left(\varrho_{I}(A)\right) .
$$

Using the operators $\Gamma^{I}$, one can construct an algebra of field operators $\mathscr{F}$ in $\mathscr{H}$. It is generated by elements

$$
\phi_{\varrho}=\Gamma^{I} U \text {. }
$$

$U$ unitaries in an associative algebra of observables $\mathscr{A}$, to which the endomorphisms $\varrho_{J}$ extend, and

$$
\varrho=\sigma_{U} \circ \varrho_{I}
$$

$\phi_{\varrho}$ is determined by $\varrho$ only up to multiplication with a unitary element in the center of the algebra of observables. It obeys

$$
\pi(A) \phi_{\varrho}=\phi_{\varrho} \pi(\varrho(A)) \text {. }
$$

If $\varrho$ is localized in $I=\operatorname{supp} \varrho$, then $\varrho(A)=A$ for $A \in I^{\prime} \cap I=\emptyset$. Therefore

$$
\pi(A) \phi_{\varrho}=\phi_{\varrho} \pi(A) \text { if } A \subset \operatorname{Lie} \overline{\mathscr{A}}\left(I^{\prime}\right), \quad I^{\prime} \cap \operatorname{supp} \varrho=\emptyset .
$$

Let $\mathscr{F}(I)$ be the subalgebra of all field operators with this property. In this way we obtain a net of local field algebras. They are relatively local to the observables. They are not relatively local to themselves or to each other. This issue will be addressed in the next sections.

The algebra of field operators acts in a Hilbert space. Therefore a *-operation is defined. From the definition of $\Gamma^{J}$ and the completeness relation for intertwiners it follows that

$$
\Gamma^{J} \Gamma^{J *}=1
$$

This implies that $\Gamma^{J *} \Gamma^{J}$ is a projection operator. $\Gamma^{1}$ has an inverse since $\varrho_{1}$ is an automorphism. If $L=0$ or $L=1$ there is only one intertwiner $\Gamma\left(\begin{array}{cc}\frac{1}{2} \\ K & L\end{array}\right)$, with $K=\frac{1}{2}$ in both cases. Thus, $\Gamma^{1 / 2}$ maps $\mathscr{H}_{0}$ bijectively into $\mathscr{H}_{1 / 2}$, and also $\mathscr{H}_{1}$ bijectively into $\mathscr{H}_{1 / 2}$. It follows that some vectors on $\mathscr{H}_{0} \oplus \mathscr{H}_{1}$ are annihilated by $\Gamma^{1 / 2}$. Thus

$$
\operatorname{ker} \Gamma^{1 / 2} \neq \emptyset \text {. }
$$

4.4. Operator Product Expansions. Here we study products of field operators in the Hilbert space $\mathscr{H}=\oplus \mathscr{H}_{J}$ (without multiplicities). We write $\pi(A)$ for the action of the observable algebra on $\mathscr{H} ; \pi$ restricts to $\pi_{J}$ on $\mathscr{H}_{J}$. In principle the product of field operators is determined by the multiplication law in the field algebra.

Let us study products of intertwiners $\Gamma$. We write $\operatorname{Hom}\left(\pi^{1}, \pi^{2}\right)$ for the set of intertwining operators between representations $\pi^{1}$ and $\pi^{2}$ of the observable 
algebra on Hilbert spaces $\mathscr{H}^{(1)}$ and $\mathscr{H}^{(2)}$,

$$
\operatorname{Hom}\left(\pi^{1}, \pi^{2}\right) \ni \Gamma: \mathscr{H}^{(1)} \rightarrow \mathscr{H}^{(2)}
$$

if

$$
\pi^{2}(\mathrm{~A}) \Gamma=\Gamma \pi^{1}(\mathrm{~A}) .
$$

The Hilbert spaces will be implied by the name of representations, for instance $\pi_{L} \circ \varrho$ acts in $\mathscr{H}_{L}$ for any endomorphism $\varrho$. The relation with the space of intertwiners $\operatorname{Hom}\left(\varrho^{\prime}, \varrho\right)$ between endomorphisms is as follows

$$
T \in \operatorname{Hom}\left(\varrho^{\prime}, \varrho\right) \text { implies } \pi(T) \in \operatorname{Hom}\left(\pi \circ \varrho^{\prime}, \pi \circ \varrho\right)
$$

for any representation $\pi$ of the observables algebra.

Consider intertwiners

$$
\Gamma\left(\begin{array}{cc}
J \\
K & L
\end{array}\right) \in \operatorname{Hom}\left(\pi_{L} \circ \varrho_{J}, \pi_{K}\right) .
$$

Given $J_{1}, J_{2}, M$, products of the form

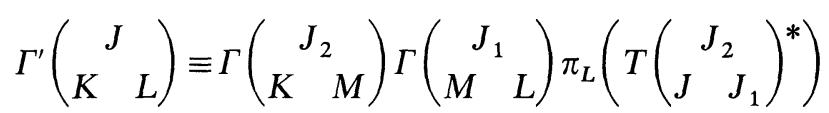

are in $\operatorname{Hom}\left(\pi_{L} \circ \varrho_{J}, \pi_{K}\right)$ if

$$
T\left(\begin{array}{c}
J_{2} \\
J
\end{array}\right) \in \operatorname{Hom}\left(\varrho_{J_{1}} \varrho_{J_{2}}, \varrho_{J}\right)
$$

It follows that

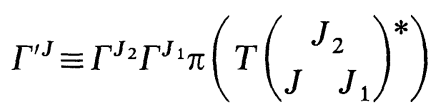

obeys

$$
\pi(A) \Gamma^{J}=\Gamma^{\prime J} \pi\left(\varrho_{J}(A)\right)
$$

just like $\Gamma^{J}$. That is, $\Gamma^{J} \in \operatorname{Hom}\left(\pi \circ \varrho_{J}, \pi\right)$. We omit symbols $\pi$ in the following. Consider now a complete set of intertwiners $T\left(\begin{array}{cc}J_{2} \\ J & J_{1}\end{array}\right)$ as described in Sect. 4.2. Label $a$ will distinguish between NS- and R-intertwiner. The completeness relation implies

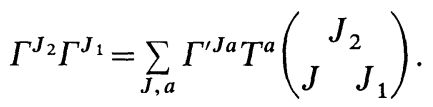

Consider now products of fields

$$
\phi^{J_{2}}=\Gamma^{J_{2}} U_{2}, \quad \phi^{J_{1}}=\Gamma^{J_{1}} U_{1}, \quad U_{i} \in \mathscr{A} .
$$

Using commutation relations of observables with constant fields $\Gamma$, we obtain

$$
\phi^{J_{2}} \phi^{J_{1}}=\sum_{J, a} \phi^{J, a}
$$

with

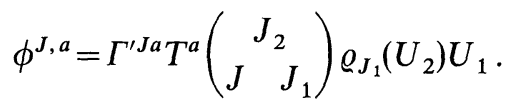


On the vacuum sector $\mathscr{H}=\mathscr{H}_{0}$, we have $\pi=\pi_{0}$ and only NS-intertwiners survive. They are unique, and we may drop the lable $a$. Moreover, uniqueness implies that the restriction of $\Gamma^{\prime}$ to $\mathscr{H}_{0}$ must be proportional to $\Gamma\left(\begin{array}{cc}J & \\ J & 0\end{array}\right)=$ restriction of $\Gamma^{J}$. Thus we have

Proposition 4.3 (Operator Product Expansion on the Vacuum). Let $\phi^{J_{i}}=\Gamma^{J_{i}} U_{i}$. Then

$$
\begin{gathered}
\phi^{J_{2}} \phi^{J_{1}}=\sum_{J} c_{J J_{2} J_{1}} \phi^{J} \quad \text { on } \mathscr{H}_{0}, \\
\phi^{J}=\Gamma^{J} U \quad \text { with } \quad U=T\left(\begin{array}{cc}
J_{2} \\
J & J_{1}
\end{array}\right) \varrho_{J_{1}}\left(U_{2}\right) U_{1} \in \mathscr{A} .
\end{gathered}
$$

If $U_{i} U_{i}^{*}=1(i=1,2)$ then $U U^{*}=1\left(\right.$ on $\left.\mathscr{H}_{0}\right)$.

Consider the algebra $\mathscr{F}$ of field operators on $\mathscr{H}$ which is generated by operators $\Gamma^{J}, J=0, \frac{1}{2}, 1$ and observables $A$. It contains all products of field operators $\phi^{J}=\Gamma^{J} U$. Using the operator product expansion one can express the vacuum expectation value $\langle 0|\phi| 0\rangle$ of any product of fields $\phi \in \mathscr{F}$ as expectation value of an observable.

This assertion follows from the fact that $\Gamma^{0}=1$ and $\Gamma^{J} \mathscr{H}_{0} \perp \mathscr{H}_{0}$ for $J \neq 0$. The structure constants $c_{J_{J_{2} J_{1}}}$ can be determined from the explicit form of the intertwiners. We leave this as an exercise to the reader.

\section{Braid Group}

5.1. Jones Algebra and Braid Group Representation Determined by the Endomorphism $\varrho_{1 / 2}$. A well known result of the theory of superselection sectors [8] says that a transportable localized endomorphism $\varrho$ of an algebra of local observables $\mathscr{A}$ determines a representation of the braid group $B_{\infty}$ by operators $\sigma_{i} \in \mathscr{A}$. More precisely there exists a unitary operator $\varepsilon_{\varrho}$ in the commutant of $\varrho^{2}(\mathscr{A})$ in $\mathscr{A}$,

$$
\varepsilon_{\varrho} \in \varrho^{2}(\mathscr{A})^{\prime} \text {, unitary }
$$

such that

$$
\sigma_{i}=\varrho^{i-1}\left(\varepsilon_{\varrho}\right), \quad i=1,2, \ldots
$$

satisfy Artin relations

$$
\begin{gathered}
\sigma_{i} \sigma_{j}=\sigma_{j} \sigma_{i} \text { if }|i-j| \geqq 2, \\
\sigma_{i} \sigma_{i+1} \sigma_{i}=\sigma_{i+1} \sigma_{i} \sigma_{i+1} .
\end{gathered}
$$

In $d \geqq 3$-dimensional space time, this braid group representation degenerates into a permutation group representation, that is $\sigma_{i}=\sigma_{i}^{-1}$.

There exists a definition of $\varepsilon_{e}$ in terms of $\varrho$ (see Introduction), but it is much easier to determine $\varepsilon_{\ell}$ from its properties. The Artin relations admit substitution of $\sigma_{i}^{-1}$ for $\sigma_{i}$. This arbitrariness is intrinsic and corresponds to the possibility of space reflections. The Artin relations also leave an arbitrary phase factor in $\varepsilon_{e}$ free. This phase factor is determined by the explicit definition, it can be recovered from the "spin statistics" theorem [9]. 
The representation of the braid group turns out to come from a Jones algebra. Following Fredenhagen, Rehren, and Schroer [8], this Jones Algebra and related quantities are defined as follows. They consider irreducible transportable localized endomorphisms in an associative observable algebra $\mathscr{A}$ with trivial center.

An endomorphism $\varrho$ is said to be conjugate to $\varrho$ if the representation $[\varrho \varrho]]=[\varrho \varrho \varrho]$ contains the vacuum representation $[i d]$. From the associative algebra of observables $\mathscr{A}$ one obtains a Jones tunnel

$$
\mathscr{A} \supseteqq \varrho(\mathscr{A}) \supseteqq \varrho \varrho(\mathscr{A}) \supseteqq \varrho \varrho \varrho(\mathscr{A}) \supseteqq \ldots
$$

and a dual Jones tower of the corresponding commutants $M_{i}$ in $\mathscr{A}$.

Consider intertwiners $R^{*}$ and $\bar{R}^{*}$ which map to the subrepresentation [id] of $[\varrho \varrho \varrho]$ and $[\varrho \varrho \bar{c}]$,

$$
\begin{array}{lll}
R^{*} \in \operatorname{Hom}(\varrho \varrho, i d) & \text { with } & R^{*} R=1, \\
\bar{R}^{*} \in \operatorname{Hom}(\varrho \varrho, i d) & \text { with } & \bar{R}^{*} \bar{R}=1,
\end{array}
$$

and the corresponding projectors

$$
\Pi=R R^{*} \text { and } \bar{\Pi}=\bar{R} R^{*} .
$$

Then the projection operators $(i=1,2, \ldots)$

$$
E_{2 i-1}=(\varrho \varrho)^{i-1}(\Pi), \quad E_{2 i}=(\varrho \varrho)^{i-1} \varrho(\bar{\Pi})
$$

obey the Jones algebra relations

$$
\begin{gathered}
E_{n} E_{m}=E_{m} E_{n}, \quad|n-m| \geqq 2, \\
E_{n} E_{n \pm 1} E_{n}=d(\varrho)^{-2} E_{n}
\end{gathered}
$$

with a parameter $d(\varrho)$ called statistical dimension. Moreover $\Phi$, defined by

$$
\Phi(A)=R^{*} \varrho(A) R
$$

is a left inverse of $\varrho$, i.e. a positive map $\mathscr{A} \rightarrow \mathscr{A}$ with $\Phi(\varrho(A))=A$ for all $A$. It is unique if $\varrho$ is irreducible (i.e. [ $\varrho]$ is irreducible) and $\varrho \circ \Phi$ defines a conditional expectation from $\mathscr{A}$ to $\varrho(\mathscr{A})$. This left inverse yields another formula for the statistical dimension

$$
\Phi\left(\varepsilon_{\varrho}\right)=\lambda_{\varrho} 1 \quad \text { with } \quad\left|\lambda_{\varrho}\right|=d(\varrho)^{-1} .
$$

The spin and statistics theorem [9] says that in a conformal field theory the phase is given by

$$
\lambda_{\varrho}=e^{2 \pi i s} d(\varrho)^{-1},
$$

where $s$ is the lowest eigenvalue of $L_{0}$ in the representation [ $\varrho$ ]. $\Phi$ also yields a Markov trace on $B_{\infty}$, i.e. link invariants [8].

A nontrivial Jones algebra, and a braid group representation which is not of the form $\sigma_{i}=\omega \cdot \mathbf{1}, \omega=$ phase factor, is only obtained when $\varrho$ is not an automorphism.

In our model, $\varrho_{0}=i d$ and $\varrho_{1}$ are automorphisms, and $\left[\varrho_{1 / 2}^{2}\right]$ contains $[i d]$. Therefore we should consider the special case

$$
\varrho=\varrho_{1 / 2}=\bar{\varrho} .
$$

$\varrho_{1 / 2}$ is not localized, but it is a limit of localized morphisms. Also, our global algebra has a nontrivial center, therefore $R^{*} R \in C \cdot \mathbf{1}$ is not automatic. But 
operators $R=\bar{R}$ which fulfill conditions (5.5) exist, and can be obtained from the intertwiners in Sect. 4,

$$
R^{*}=R_{\mathrm{NS}}^{*}+R_{\mathrm{R}}^{*}
$$

with corresponding projection operators

$$
\begin{gathered}
\Pi=\Pi_{0}+\bar{\Pi}_{0}=b_{1 / 2} b_{-1 / 2} Y_{-}+\frac{1}{2}\left(1+\sqrt{2} b_{0}\left[b_{1}-b_{-1}\right]\right) Y_{+}, \\
Y_{ \pm}=\frac{1}{2}[1 \pm Y] .
\end{gathered}
$$

From this one may now compute the Jones projectors.

$$
\begin{gathered}
E_{2 i+1}=b_{i+1 / 2} b_{-i-1 / 2} Y_{-}+\frac{1}{2}\left[1+\left(b_{i}+b_{-i}\right)\left(b_{i+1}-b_{-i-1}\right)\right] Y_{+} \text {for } i \neq 0, \\
E_{2 i+2}=b_{i+1} b_{-i-1} Y_{+}+\frac{1}{2}\left[1-\left(b_{i+1 / 2}-b_{-i-1 / 2}\right)\left(b_{i+3 / 2}+b_{-i-3 / 2}\right)\right] Y_{-} \\
E_{1}=b_{1 / 2} b_{-1 / 2} Y_{-}+\frac{1}{2}\left(1+\sqrt{2} b_{0}\left[b_{1}-b_{-1}\right]\right) Y_{+}
\end{gathered}
$$

In the vacuum representation the result simplifies since $Y_{-}=1, Y_{+}=0$ on $\mathscr{H}_{0}$,

$$
\begin{gathered}
E_{2 i+1}=b_{1 / 2+i} b_{-1 / 2-i} \\
E_{2 i+2}=\frac{1}{2}\left[1-\left(b_{1 / 2+i}-b_{-1 / 2-i}\right)\left(b_{3 / 2+i}+b_{-3 / 2-i}\right)\right]
\end{gathered}
$$

$(i=0,1,2, \ldots)$. Similar formulae for Jones projectors in terms of complex fields were obtained in Connes and Evans [29]. The $E_{n}$ satisfy the relations of a TemperlyLieb Jones algebra with

$$
d\left(\varrho_{1 / 2}\right)=\sqrt{2} .
$$

When $\varrho=\bar{\varrho}$, the formulae for the Jones algebra and the braid group generators look very similar, and one can obtain one from the other $[8,22]$.

$$
\varepsilon_{\varrho}=\gamma((1+i) \Pi-1)=\gamma\left((1+i) E_{1}-1\right)
$$

with $\Pi$ as defined above, for $\varrho=\varrho_{1 / 2}$. More generally

$$
\sigma_{n}=\gamma\left((1+i) E_{n}-1\right)
$$

for $d(\varrho)=\sqrt{2} ; \gamma$ is an arbitrary phase factor.

Evaluating $\Phi\left(\varepsilon_{0}\right)$ in the vacuum sector yields

$$
\Phi\left(\varepsilon_{\varrho}\right)=\frac{1}{2} \gamma(-1+i)=\lambda_{\varrho} \cdot 1 \quad\left(\varrho=\varrho_{1 / 2}\right) .
$$

Therefore $d(\varrho)=\sqrt{2}$ in agreement with the previous result. According to the spin and statistics theorem, the preferred choice for the statistical phase $\omega_{\varrho}=\lambda_{\varrho} /\left|\lambda_{\varrho}\right|$ is

$$
\omega_{\varrho}=e^{2 \pi i s}
$$

$s=$ lowest eigenvalue of $L_{0}$ in the representation [@]. Here $s=\frac{1}{16}$ and we obtain

$$
\gamma=-e^{i \frac{3 \pi}{8}}
$$

We write $\varepsilon_{1 / 2}$ for $\varepsilon_{\varrho}$ if $\varrho=\varrho_{1 / 2} . \varepsilon_{1 / 2}$ acts by right multiplication on $\mathscr{A}$. Its eigenspaces are generated by projectors in the commutant of $\varrho_{1 / 2}^{2}(\mathscr{A})$. Projectors $\Pi_{0}, \Pi_{1}=\frac{1}{2}(1+Y)-\Pi_{0}, \bar{\Pi}_{0}$ and $\bar{\Pi}_{1}$ are orthogonal and sum to 1 . Thus

$$
\varepsilon_{1 / 2}=\gamma((1+i) \Pi-1), \quad \Pi=\Pi_{0}+\bar{\Pi}_{0}
$$


obeys

$$
\Pi_{0} \varepsilon_{1 / 2}=i \gamma \Pi_{0}, \quad \Pi_{1} \varepsilon_{1 / 2}=-\gamma \Pi_{1}, \quad \text { etc. }
$$

Therefore we obtain that the eigenvalues of $\varepsilon_{1 / 2}$ are $i \gamma$ and $-\gamma, \gamma=-e^{i \frac{3 \pi}{8}}$. Our results are consistent with the general classification of Longo [30].

According to Jones, the possible values of $d(\varrho)$ are given by $d(\varrho) \geqq 2$ or

$$
d(\varrho)=2 \cos \left(\frac{\pi}{k}\right),
$$

$k$ a natural integer $\geqq 3$. The value of $\sqrt{2}$ corresponds to

$$
k=4 \text {. }
$$

In our model, $\varrho_{0}=i d$ and $\varrho_{1}$ are automorphisms, therefore

$$
d\left(\varrho_{0}\right)=1=d\left(\varrho_{1}\right)
$$

and

$$
\varepsilon_{1}=-1
$$

by the spin and statistics theorem.

For reducible representations $\left[\varrho_{I_{1}} \ldots \varrho_{I_{n}}\right]$ one defines

$$
d\left(\varrho_{I_{1}} \ldots \varrho_{I_{n}}\right)=d\left(\varrho_{I_{1}}\right) \ldots d\left(\varrho_{I_{n}}\right) .
$$

The general theory says that they obey sum rules [8]. If $\varrho=\varrho_{I_{1}} \ldots \varrho_{I_{n}}$ obeys

$$
[\varrho]=\sum N_{K}\left[\varrho_{K}\right],
$$

then

$$
d(\varrho)=\sum N_{K} d\left(\varrho_{K}\right) .
$$

Comparison with our fusion rules shows that these sum rules are indeed satisfied. It suffices to check this for products of two endomorphisms. Indeed

$$
\begin{gathered}
(\sqrt{2})^{2}=d\left(\varrho_{1 / 2}^{2}\right)=d\left(\varrho_{0}\right)+d\left(\varrho_{1}\right)=1+1, \\
1 \cdot \sqrt{2}=d\left(\varrho_{1} \varrho_{1 / 2}\right)=d\left(\varrho_{1 / 2}\right)=\sqrt{2}, \\
1 \cdot 1=d\left(\varrho_{1}^{2}\right)=d\left(\varrho_{0}\right)=1 .
\end{gathered}
$$

We saw earlier that our endomorphisms $\varrho$ come from lifting partial isometries $V$ which can be classified by their index. By comparism we see that

$$
d(\varrho)=2^{-\frac{1}{4} \operatorname{index}(V)} .
$$

This holds both for irreducible and reducible representations $\varrho=\varrho_{I_{1}} \ldots \varrho_{I_{n}}$ since

$$
\text { index } V_{I_{1}} \ldots V_{I_{n}}=\sum_{j} \operatorname{index} V_{I_{j}} \text {. }
$$

The sum rule for statistical dimensions translates into an remarkable sum rule for indices

$$
2^{-\frac{1}{4} \sum \text { index } V_{I_{J}}}=\sum_{K} N_{K} 2^{-\frac{1}{4} \text { index } V_{K}} .
$$

Following Jones and Longo $[22,30], d(\varrho)^{-2}$ can be interpreted as index of the inclusion $\varrho(\mathscr{A}) \subset \mathscr{A}$ of associative algebras. 
Let us finally note that the whole Lie algebra of observables Lie $\overline{\mathscr{A}}$ can be reconstructed from the Jones projectors $E_{n}$ and the central elements $Y_{ \pm}=\frac{1}{2}[1 \pm Y]$. It suffices to exhibit

$$
b_{c} b_{a}=\hat{J}_{c a}=J_{c a}+\frac{1}{4}\left[1+(-1)^{2 a} Y\right] \delta_{a,-c}
$$

for $a>0, c= \pm(a-1)$. The other operators $J_{c a}$ can be obtained by considering adjoints and commutators of these, using $J_{a b}=-J_{b a}$. The generators $L_{n}$ and $N_{n}$ are infinite sums of operators $b_{a} b_{c}$ by Eqs. (2.41)f.

One finds by explicit computation

$$
\begin{gathered}
b_{c} b_{c+1}= \pm 2\left(E_{2 c}-\frac{1}{2}\right) E_{2 c+2} E_{2 c+1} Y_{ \pm} \\
b_{-c} b_{c+1}=2 E_{2 c+2} E_{2 c+1}\left(E_{2 c}-\frac{1}{2}\right) Y_{+} \\
b_{c} b_{-c}=E_{2 c},
\end{gathered}
$$

for $c \geqq \frac{1}{2}$, while

$$
\begin{gathered}
b_{0} b_{1}=\sqrt{2} E_{2}\left(E_{1}-\frac{1}{2}\right) Y_{ \pm} \\
b_{0} b_{-1}=\sqrt{2}\left(E_{1}-\frac{1}{2}\right) E_{2} Y_{+} \\
b_{0}^{2}=\frac{1}{2} Y_{+} .
\end{gathered}
$$

\pm is to be read as $(-1)^{2 c}$.

5.2. Standard Braid Matrices. Following Fredenhagen, Rehren, and Schroer [8], standard braid matrices are defined by the action of the braid group $B_{N}$ on products of at least $N+1$ intertwiners by right multiplication, as follows.

We will only consider intertwiners which are nonvanishing in the vacuum representation (NS-intertwiners). These are unique in our model, and we regard them as operators in $\mathscr{H}_{0}$, omitting symbols $\pi_{0}$.

Fix $J$ and let $\operatorname{Path}_{K_{n} K_{0}}^{n}$ be the set of sequences of $n+1$ sector labels

$$
\xi=\left(K_{n}, K_{n-1} \ldots K_{1}, K_{0}\right) \text {. }
$$

Set

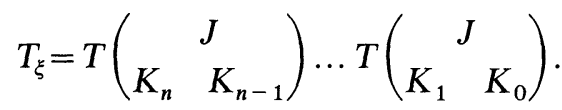

It follows from the intertwining property of $T\left(\begin{array}{cc}J \\ K & L\end{array}\right)$ that

$$
T_{\xi} \in \operatorname{Hom}\left(\varrho_{K_{0}} \varrho_{J}^{n}, \varrho_{K_{n}}\right) .
$$

Thus, $T_{\xi}$ are isometric maps $\mathscr{H}_{0} \rightarrow \mathscr{H}_{0}$. $T_{\xi}$ vanishes on the orthogonal complement of a subrepresentation of $\pi_{0} \circ \varrho_{K_{0}} \varrho_{K}^{n}$ which is equivalent to $\pi_{0} \circ \varrho_{K_{n}}$. $T_{\xi}$ with $\xi \in \operatorname{Path}_{K_{n} K_{0}}^{n}$ is a basis in the space Hom $\left(\varrho_{K_{0}} \varrho_{J}^{n}, \varrho_{K_{n}}\right)$ of intertwiners. This is because one may reduce the representation $\pi_{0}{ }^{\circ} \varrho_{K_{0}} \varrho_{J}^{n}$ in steps. First reduce the representation $\pi_{0} \circ \varrho_{K_{0}} \varrho_{J}$ of $\mathscr{A}$ into irreducibles $\pi_{0} \circ \varrho_{K_{1}}$, using $T\left(\begin{array}{cc}J \\ K_{1} & K_{0}\end{array}\right)$. Then restrict this representation to $\varrho_{J}(\mathscr{A})$. This restriction may be reducible. Reduce this representation $\pi_{0} \circ \varrho_{K_{1}} \varrho_{J}$ of $\mathscr{A}$ into irreducibles $\pi_{0} \circ \varrho_{K_{2}}$ using $T\left(\begin{array}{c}J \\ K_{2}\end{array} \quad K_{1}\right)$, and so
on. 
Now we remember that

$$
\left[\varepsilon^{J}, A\right]=0 \text { for } A \in \varrho_{J}^{2}(A) .
$$

It follows from this together with the intertwining properties of $T$ 's and the definition $\sigma_{i}=\varrho^{i-1}\left(\varepsilon_{J}\right)$ of the braid group generators that

$$
T_{\xi} \varrho_{K_{0}}\left(\sigma_{i}\right) \in \operatorname{Hom}\left(\varrho_{K_{0}} \varrho_{J}^{n}, \varrho_{K_{n}}\right) \text { for } i \leqq n .
$$

Therefore this must be a linear combination of $T_{\xi^{\prime}}$ with $\xi^{\prime} \in \operatorname{Path}_{K_{n} K_{0}}^{n}$. More generally, for any element $b=\sigma_{i_{1}} \ldots \sigma_{i_{M}} \in B_{n}$, viz. $i_{1}, \ldots, i_{M} \leqq n$,

$$
T_{\xi} b=\sum_{\xi^{\prime}} T_{\xi^{\prime}} R_{\xi}^{\xi^{\prime}}(b)
$$

i.e. we get a representation of the braid group $B_{n}$ on $n$ threads by matrices $R_{\xi}^{\xi^{\prime}}$, $\xi, \xi^{\prime} \in \operatorname{Path}_{K_{n} K_{0}}^{n}$. Of course $R$ depends on $J$.

Making the identification $\pi \circ \varrho_{K} \equiv \pi_{K}$ these identities get translated into similar identities for intertwiners $\Gamma\left(\begin{array}{cc}J \\ K & L\end{array}\right)$. Using the standard abbreviation

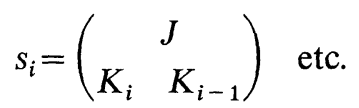

we get in the special case $n=2$

$$
\Gamma\left(s_{2}\right) \Gamma\left(s_{1}\right) \varepsilon_{J}=\sum_{s_{2}^{\prime} s_{1}^{\prime}} \Gamma\left(s_{2}^{\prime}\right) \Gamma\left(s_{1}^{\prime}\right) R_{s_{2} s_{1}}^{s_{2}^{\prime} s_{1}^{\prime}} .
$$

Since we know $\varepsilon_{J}$ and the intertwiners explicitly, the matrix $R$ can be calculated. One writes

$$
R_{s_{2} s_{1}}^{s_{1}^{\prime} s_{1}^{\prime}} \equiv \delta_{K_{2} K_{1}^{\prime}} \delta_{K_{0} K_{0}^{\prime}} C_{K_{1} K_{1}^{\prime}}\left[\begin{array}{cc}
J & J \\
K_{2} & K_{0}
\end{array}\right]_{\text {phys }} .
$$

$J=\frac{1}{2}$ is the interesting case, because automorphisms like $\varrho_{1}$ lead to 1-dimensional braid group representations.

The result is given explicitly in Table 1 (Appendix B). It agrees with the following expression involving $6 j$-symbols for $U_{q}(\operatorname{sl}(2))$ when $q=e^{-i \pi / 2}, J_{2}=J_{3}=J$,

$$
\begin{aligned}
C_{J J^{\prime}}\left[\begin{array}{ll}
J_{2} & J_{3} \\
J_{1} & J_{4}
\end{array}\right]_{\mathrm{phys}} & =e^{i \frac{\pi}{2}} C_{J J^{\prime}}\left[\begin{array}{ll}
J_{2} & J_{3} \\
J_{1} & J_{4}
\end{array}\right]_{q} \\
& =(-1)^{J+J^{\prime}-J_{1}-J_{4}} q^{\left(c_{J_{1}}+c_{J_{4}}-c_{J}-c_{J^{\prime}}\right) / 2} e^{i \frac{\pi}{2}}\left\{\begin{array}{lll}
J_{2} & J_{1} & J \\
J_{3} & J_{4} & J^{\prime}
\end{array}\right\}_{q} . \\
c_{J} & =J(J+1) .
\end{aligned}
$$

Let us note that the result for $R_{. .}$depends on a phase convention. The intertwiners $T\left(\begin{array}{cc}J \\ K & L\end{array}\right)$ are only determined up to phase factors. It turns out that a particular ratio of these phase factors enters into the result for the braid matrices. We have chosen these phase factors in a particular way. 


\section{Fields with Quantum Symmetry $U_{q}(s l(2)), q=e^{-i \pi / 2}$}

6.1. The Quantum Group $U_{q}(s l(2))$ with $q=-i-a$ Brief Review. The quantum group algebra $[14,31,38,33] U_{q}(s l(2))$ is the associative algebra $\mathscr{G}$ with unit $\mathbf{1}$, generators $S_{+}, S^{-}, q^{ \pm S_{z} / 2}$ and relations

A coproduct

$$
\begin{aligned}
& q^{S_{z} / 2} S_{ \pm} q^{-S_{z} / 2}=q^{ \pm 1 / 2} S_{ \pm}, \quad q^{S_{z} / 2} q^{-S_{z} / 2}=1=q^{-S_{z} / 2} q^{S_{z} / 2}, \\
& {\left[S_{+}, S_{-}\right]=\frac{q^{S_{z}}-q^{-S_{z}}}{q^{1 / 2}-q^{-1 / 2}} \text {. }}
\end{aligned}
$$

$$
\Delta: \mathscr{G} \rightarrow \mathscr{G} \otimes \mathscr{G}
$$

is defined by $\Delta(\mathbf{1})=1 \otimes 1$ and

$$
\begin{aligned}
\Delta\left(q^{ \pm S_{z} / 2}\right) & =q^{ \pm S_{z} / 2} \otimes q^{ \pm S_{z} / 2}, \\
\Delta\left(\mathbf{S}_{ \pm}\right) & =q^{S_{z} / 2} \otimes S_{ \pm}+S_{ \pm} \otimes q^{-S_{z} / 2} .
\end{aligned}
$$

A counit $\varepsilon: \mathscr{G} \rightarrow \mathbf{C}$ is defined by $\varepsilon(\mathbf{1})=1$ and

$$
\varepsilon\left(q^{ \pm S_{z} / 2}\right)=1, \quad \varepsilon\left(S_{ \pm}\right)=0 .
$$

Finally there is an antipode

$$
\begin{aligned}
\mathscr{S}\left(q^{ \pm S_{z} / 2}\right) & =q^{\mp S_{z} / 2}, \\
\mathscr{S}\left(S_{ \pm}\right) & =-q^{ \pm 1 / 2} S_{ \pm} .
\end{aligned}
$$

$\Delta$ is a homomorphism of algebras, $\varepsilon$ a homomorphism of $\mathscr{G}$ into $\mathbf{C}$, and $\mathscr{S}$ is an antiautomorphism of $\mathscr{G}$, such that

$$
(i d \otimes \varepsilon) \cdot \Delta=i d .
$$

Given representations $\tau^{J}$ and $\tau^{K}$ of the algebra $\mathscr{G}$ on vector spaces $V^{J}$ and $W^{K}$, a representation $\tau$ on the tensor product $V^{J} \otimes W^{K}$ is defined as follows. Write the coproduct in the form

$$
\Delta(X)=\sum_{Y, Z} a(X \mid Y, Z) Y \otimes Z .
$$

Then

$$
\tau(X) v \otimes w=\sum_{Y, Z} a(X \mid Y, Z) \tau^{J}(Y) v \otimes \tau^{K}(Z) w .
$$

The quantum dimension (character) of a representation $\tau$ is defined by

$$
d_{\tau}=\operatorname{tr} \tau\left(q^{S_{z}}\right) .
$$

For $q=-i$, the group $U_{q}(\operatorname{sl}(2))$ has 3 "physical representations" labelled by isospin $J=0, \frac{1}{2}, 1$ of dimension $2 J+1$ and quantum dimension $d_{J}=1, \sqrt{2}, 1$. Physical representations are those which are irreducible and have strictly positive quantum dimension. We write $[J]$ for the equivalence class of the $2 J+1$ dimensional physical representation.

The tensor product of physical representations decomposes into physical representations, plus representations of quantum dimension 0 [38]. Omitting the latter, the decomposition

$$
[J] \otimes[K]=\oplus N_{L}^{J K}[L]
$$


is explicitly given by $[0] \otimes[J]=[J]$ and

$$
\begin{aligned}
& {\left[\frac{1}{2}\right] \otimes\left[\frac{1}{2}\right]=[0]+[1],} \\
& {\left[\frac{1}{2}\right] \otimes[1]=\left[\frac{1}{2}\right],} \\
& {[1] \otimes[1]=[0] .}
\end{aligned}
$$

This holds whenever $q^{4}=1$. The quantum dimension satisfies the sum rule

$$
d_{J} d_{K}=\sum_{L} N_{L}^{J K} d_{L}
$$

If $e_{j}^{J}$ and $e_{k}^{K}$ are basis vectors in physical representation spaces, then their tensor product decomposes into basis vectors in the physical subrepresentations

$$
e_{j}^{J} \otimes e_{k}^{K}=\sum_{L, l} e_{l}^{L}\left[\begin{array}{lll}
L & J & K \\
l & j & k
\end{array}\right],
$$

plus contributions from subrepresentations with quantum dimension 0 . This decomposition involves Clebsch Gordan coefficients.

Representations $\tau^{J} \otimes \tau^{K}$ and $\tau^{K} \otimes \tau^{J}$ are equivalent, and there exists a map $\mathscr{R}$

$$
\mathscr{R}: V^{J} \otimes W^{K} \rightarrow W^{K} \otimes V^{J}
$$

which commutes with the action of the quantum group algebra. In terms of basis vectors

$$
\mathscr{R} e_{j}^{J} \otimes e_{k}^{K}=\sum_{m, n} e_{m}^{K} \otimes e_{n}^{J} \mathscr{R}_{j k}^{m n} .
$$

Of course, $\mathscr{R}$ depends on $J$ and $K$. Expressions for quantum group $\mathscr{R}$-matrices can be found in the literature. For generic $q, 6 j$-symbols can be thought to be defined through Eq. (6.52) (see below), without the phase factor $e^{i \pi / 2}$, with unrestricted sum over $J_{12}$. When $q$ becomes a root of unity, $6 j$-symbols for unphysical $J_{12}$ become zero, assuming standard normalisation conventions, but some "unphysical" Clebsch Gordan coefficients become infinite.

6.2. Field Transformation Law. If we substitute quantum group representations $[J]$ for representations $\left[\varrho_{J}\right]$ of the observable algebra (i.e. for superselection sectors) then the fusion rules in Theorem 4 correspond precisely to the tensor product decomposition rules (6.14) for $U_{q}(s l(2))$ with $q^{4}=1$. Also the statistical dimensions $d\left(\varrho_{J}\right)$ of the representations $\left[\varrho_{J}\right]$ agree with the quantum dimensions $d_{J}$ of representations $[J]$, see Theorem 2 and Sect. 5 .

This suggests that states in the superselection sector $\mathscr{H}_{J}$ should transform according to the $2 J+1$-dimensional representation of $U_{q}(\operatorname{sl}(2))$, with $q$ some $4^{\text {th }}$ root of unity. The analogy with gauge groups in $d \geqq 3$-dimensional quantum field theories described in the introduction suggests that observables should be quantum group invariant. This implies that the irreducible representation $\pi_{J}$ of the algebra of observables must occur with multiplicity $2 J+1$ (at least) and we are led to consider the Hilbert space

$$
\mathscr{H}_{\text {phys }}=\bigoplus_{J=0, \frac{1}{2}, 1} \bigoplus_{m=-J}^{J} \mathscr{H}_{J m},
$$

where $\mathscr{H}_{J m}$ are $2 J+1$ copies of the same representation space carrying representation $\pi_{J} \cong \pi_{0} \circ \varrho_{J}$. The quantum group generators become operators $X$ in the 
Hilbert space $\mathscr{H}_{\text {phys }}$ which commute with observables $A$

$$
[X, A]=0 \text { for all } X \in \mathscr{G}, A \in \mathscr{A} \text {. }
$$

The quantum group algebra $\mathscr{G}$ is to be regarded as the generalization of the universal enveloping algebra of a Lie algebra, i.e. $X$ behaves like a sum of products of Lie group generators $x, y, \ldots$ in many respects. When one studies the transformation law of states and fields under elements of the universal enveloping algebra of an ordinary Lie algebra of symmetries, one obtains formulae which look precisely like those which we are going to write down for the quantum group case. In the Lie algebra case, comultiplication is

$$
\begin{gathered}
\Delta(x)=1 \otimes x+x \otimes 1, \\
\Delta(x y)=1 \otimes x y+x y \otimes 1+x \otimes y+y \otimes x
\end{gathered}
$$

etc., and $\varepsilon(\mathbf{1})=1, \varepsilon\left(X_{1} \ldots X_{n}\right)=0$.

Accordingly the proper definition of invariance of the vacuum is

$$
X|0\rangle=|0\rangle \varepsilon(X) .
$$

Let $i_{J m}^{*}: \mathscr{H}_{0} \rightarrow \mathscr{H}_{J m}$ be the identification map which identifies representations $\left(\pi_{0} \circ \varrho_{J}, \mathscr{H}_{0}\right)$ and $\left(\pi_{J}, \mathscr{H}_{J m}\right)$. The natural action of $X \in \mathscr{G}$ on states in $\mathscr{H}_{\text {phys }}$ is given by

$$
X i_{J m}^{*}|\psi\rangle=\sum_{n} i_{J n}^{*}|\psi\rangle \tau_{n m}^{J}(X), \quad|\psi\rangle \in \mathscr{H}_{0} .
$$

This commutes with the action of observables. Finally, we would like to introduce multiplets of field operators $\phi_{m}^{J}$ which create states in $\mathscr{H}_{\text {phys }}$ from the vacuum. Following Buchholz, Mack, and Todorov [17], the transformation law of pointlike fields is postulated to be given by the following generalized commutation relations

$$
X \phi_{m}^{J}(z)=\sum_{Y, Z} a(X \mid Y, Z) \sum_{n} \phi_{n}^{J}(z) \tau_{n m}^{J}(Y) Z .
$$

Factors $a(\ldots)$ are given by the comultiplication law (6.10). Since $(i d \otimes \varepsilon) \Delta=i d$, it follows from this that states $\phi_{m}^{J}(z)|\psi\rangle\left(|\psi\rangle \in \mathscr{H}_{0} \subset \mathscr{H}_{\text {phys }}\right)$ transform according to the $2 J+1$-dimensional representation

$$
X \phi_{m}^{J}(z)|\psi\rangle=\sum_{n} \phi_{n}^{J}(z)|\psi\rangle \tau_{n m}^{J}(X) \quad \text { for } \quad|\psi\rangle \in \mathscr{H}_{0} .
$$

This translates into the present framework as follows. The bounded field operators shall be of the form

$$
\phi_{m}^{J}=\Gamma_{m}^{J} U \text { with } \quad U \in \mathscr{A}
$$

with

$$
A \Gamma_{m}^{J}=\Gamma_{m}^{J} \varrho_{J}(A) \text { for all } m=-J \ldots J .
$$

We may put $\Gamma_{0}^{0}=1$ so that fields $\phi_{m}^{J}$ are observables for $J=0$. Assuming $U U^{*}=1$ we obtain from Eq. (6.28),

$$
A \phi_{m}^{J}=\phi_{m}^{J} \varrho(A), \quad \varrho=\sigma_{U} \varrho_{J} .
$$

The localization properties of such a field, which substitute for the $z$-dependence in the pointlike fields $\phi_{m}^{J}(z)$, are determined by the observable factor $U$, for given $J$. Observables are quantum group invariant, and the factors $\Gamma_{m}^{J}$ are required to 
transform according to a $2 J+1$-dimensional representation in the sense that

$$
X \Gamma_{m}^{J}=\sum_{Y, Z} a(X \mid Y, Z) \sum_{n} \Gamma_{n}^{J} \tau_{n m}^{J}(Y) Z .
$$

As a consequence, the same generalized commutation relations are valid for fields $\phi_{m}^{J}=\Gamma_{m}^{J} U, U \in \mathscr{A}$, since $[X, U]=0$. Explicitly

$$
\begin{gathered}
q^{ \pm S_{z} / 2} \phi_{M}^{J}=\sum_{n} \phi_{n}^{J} \tau_{n m}^{J}\left(q^{ \pm S_{z} / 2}\right) q^{ \pm S_{z} / 2} \\
\mathrm{~S}_{ \pm} \phi_{m}^{J}=\sum_{n}\left\{\phi_{N}^{J} \tau_{n m}^{J}\left(q^{S_{z} / 2}\right) S_{ \pm}+\phi_{n}^{J} \tau_{n m}^{J}\left(S_{ \pm}\right) q^{-S_{z} / 2}\right\}
\end{gathered}
$$

This determines the transformation law of states $\left(\phi_{m}^{J, a} \equiv \Gamma_{m}^{J} U_{a}\right)$,

$$
\phi_{m_{n}}^{J_{n}, n} \ldots \phi_{m_{1}}^{J_{1}, 1}|0\rangle \text {. }
$$

One pushes the generators of $\mathscr{G}$ through fields, using the generalized commutation relations, until they act on the vacuum, where they get converted into a complex factor $\varepsilon(X)$. It follows that such states transform according to the tensor product

$$
V^{J_{n}} \otimes \ldots \otimes V^{J_{1}}
$$

of $2 J_{i}+1$-dimensional physical representations $\left(\tau^{J_{i}}, V^{J_{i}}\right)$, or a subrepresentation of it.

The transformation law of fields can be inverted,

$$
\begin{gathered}
\phi_{m}^{J} q^{ \pm S_{z} / 2}=\sum_{n} q^{ \pm S_{z} / 2} \phi_{n}^{J} \tau_{n m}^{J}\left(q^{\mp S_{z} / 2}\right), \\
\phi_{m}^{J} S_{ \pm}=\sum_{n}\left\{S_{ \pm} \phi_{n}^{J} \tau_{n m}^{J}\left(q^{-S_{z} / 2}\right)+q^{-S_{z} / 2} \phi_{m}^{J} \tau_{n m}^{J}\left(-q^{ \pm 1 / 2} S_{\mp}\right)\right\} .
\end{gathered}
$$

This involves matrix elements of antipodes $\tau^{J}(S(Z))$, viz. $q^{\mp S_{z} / 2}=\mathscr{S}\left(q^{ \pm S_{z} 2}\right)$ and $-q^{ \pm 1 / 2} S_{ \pm}=\mathscr{S}\left(S_{ \pm}\right)$.

The inverted form (6.34) of the transformation law can be used to push generators to the left. Let us assume that the vacuum ket vector $<0$ 어 is defined as a linear form on the state space in which field algebra and quantum group act, and is invariant under the quantum group in the sense that

$$
\langle 0|X=\bar{\varepsilon}(x)<0| .
$$

Then it follows from the transformation law of fields and the invariance of the vacuum that correlation functions

$$
\left\langle 0\left|\phi_{m_{n}}^{J_{n}, n} \ldots \phi_{m_{1}}^{J_{1}, 1}\right| 0\right\rangle
$$

are quantum group invariant.

6.3. Quantum Group Covariant Field Algebras. Let $\mathscr{F}_{\text {free }}$ be the associative algebra with unit, with generators $\Gamma_{m}^{J}, J=0, \frac{1}{2}, 1, m=-J \ldots J$, and relations given by the multiplication law in the algebra of observables $\mathscr{A}$

$$
A \Gamma_{m}^{J}=\Gamma_{m}^{J} \varrho_{J}(A),
$$

and $\Gamma_{0}^{0}=1$. A general element of $\mathscr{F}_{\text {free }}$ has the form $\Gamma_{m_{n}}^{J_{n}} \ldots \Gamma_{m_{1}}^{J_{1}} A$ with $A \in \mathscr{A}, n \geqq 0$, and any product of elements may be brought into this form by use of Eq. (6.37).

This algebra is quantum group covariant in the sense that we may add the generators $X$ to those of $\mathscr{F}_{\text {free }}$, and impose generalized commutation relations (6.20), (6.30). They are consistent with the relations in the algebra $\mathscr{\mathscr { H }}_{\text {free }}$. 
It is not clear, however, whether this algebra can act in the Hilbert space $\mathscr{H}_{\text {phys}}$, defined in (6.19). If it can act the resulting representation of $\mathscr{F}_{\text {free }}$ is certainly not faithful, since a faithful representation space will include states that transform like arbitrary vectors in the tensor product $V^{J_{n}} \otimes \ldots \otimes V^{J_{1}}$ of physical representation spaces, including vectors in the unphysical subrepresentations with quantum dimension 0 . Therefore one will seek to divide by an ideal in $\mathscr{F}_{\text {free }}$, that is impose further relations in the algebra.

It would be desirable to have the relation

$$
\Gamma_{k}^{J} \Gamma_{l}^{J} \varepsilon_{J}=\sum_{m, n} \Gamma_{m}^{J} \Gamma_{n}^{J} \mathscr{R}_{k l}^{m n}
$$

with the matrix

$$
\mathscr{R}: V^{J} \otimes V^{J}=V^{J} \otimes V^{J}
$$

whose action commutes with the action of the quantum group algebra

$$
\Delta(X) \mathscr{R}=\mathscr{R} \Delta(X) \text { for all } X \in \mathscr{G} \text {. }
$$

As we explained in the introduction, desirability of such a relation comes from the fact that it yields quantum group covariant braid relations for the field operators of equal charge $J, \varepsilon_{J}$ is the unitary element in the commutant of $\varrho^{2}(\mathscr{A})$ which determines the braid group representation by operators in $\mathscr{A}$.

Let us discuss consistency of such a relation (6.38). Consistency with quantum group covariance follows from property (6.40) of $\mathscr{R}$. The fact that $\varepsilon_{J}$ determines a braid group representation implies that the same must be true for $\mathscr{R}$, therefore $\mathscr{R}$ must satisfy Yang Baxter equations. The quantum group $\mathscr{R}$-matrix satisfies these requirements [14]. Also $\varepsilon_{J}$ and $\mathscr{R}$ must have the same eigenvalues.

The eigenspaces of $\varepsilon_{J}$ in $\mathscr{A}$ are of the form $\Pi \mathscr{H}_{0}$, where $\Pi$ are projectors in the commutant of $\varrho_{J}^{2}(\mathscr{A})$. For $J=1$, this commutant consists only of the center of Lie $\mathscr{A}$, which is spanned by the projectors $\frac{1}{2}(1 \pm Y)$. The interesting case is $J=\frac{1}{2}$. The eigenvalues of $\varepsilon_{1 / 2}$ were found in Sect. 5.1 to be

$$
i \gamma \text { and }-\gamma \text { with } \gamma=-e^{i \frac{3 \pi}{8}} \text {. }
$$

This agrees with the eigenvalues of the quantum group $\mathscr{R}$-matrix of $U_{q}(\operatorname{sl}(2))$ for $q=e^{-i \pi / 2}$, for proper choice of overall phase. The overall phase is not determined by Yang Baxter equations and commutativity with $\Delta$.

Let us now try to construct operators $\Gamma_{j}^{1 / 2}$ which act in $\mathscr{H}_{\text {phys. }}$ The commutation relation (6.28) with observables is equivalent to the requirement that the transition operators $\mathscr{H}_{K k} \rightarrow \mathscr{H}_{L l}$ determined by $\Gamma_{j}^{J}$ must be proportional to the intertwiners constructed in Sect. 4. Thus

$$
\Gamma_{m}^{J}=\sum_{K, L} \sum_{k, l} C\left[\begin{array}{ccc}
K & J & L \\
k & m & l
\end{array}\right] i_{K k}^{*} \pi_{0}\left(T\left(\begin{array}{cc}
J \\
K & L
\end{array}\right)\right) i_{L l}
$$

with complex coefficients $C\left[\begin{array}{l}\cdots \\ \cdots\end{array}\right]$ that vanish unless $m+l=k$.

Consistency with transformation law (6.24), (6.30) under the quantum group requires that the coefficients $C\left[\begin{array}{l}\ldots \\ \ldots\end{array}\right]$ must be quantum group Clebsch Gordan 
coefficients, apart from a normalization factor,

$$
C\left[\begin{array}{ccc}
K & J & L \\
k & m & l
\end{array}\right]=c_{K J L}\left[\begin{array}{ccc}
K & J & L \\
k & m & l
\end{array}\right] .
$$

In this way we obtain a quantum group covariant field algebra which acts on the Hilbert space $\mathscr{H}_{\text {phys }}$. The scalar product in $\mathscr{H}_{\text {phys }}$ determines the meaning of adjoint operators. We could therefore extend our algebra of field operators to a *-algebra of operators on $\mathscr{H}_{\text {phys }}$, if we wished to include adjoints. The adjoint of quantum group generators is defined by

$$
S_{ \pm}^{*}=S_{\mp}, \quad\left(q^{ \pm S_{z} / 2}\right)^{*}=q^{\mp S_{z} / 2} .
$$

But the adjoint operators $\Gamma_{m}^{J *}$ do not have the same covariance properties as $\Gamma_{m}^{J}$. Instead

$$
\Gamma_{m}^{J *} A=\varrho_{J}(A) \Gamma_{m}^{J *}
$$

and the generalized commutation relations of $\Gamma_{m}^{J *}$ with the quantum group generators $X$ are obtained by taking the adjoint of Eq. (6.34).

$$
X \Gamma_{m}^{J *}=\sum \bar{a}\left(X^{*} Y^{*} \mid Z^{*}\right) \sum_{n} \Gamma_{n}^{J *} \bar{\tau}_{n m}^{J}\left(\mathscr{S}\left(Y^{*}\right)\right) Z
$$

( - is complex conjugate, $\mathscr{S}$ is the antipode).

Let us finally check whether condition (6.38) which assures local braid relations of fields $\phi_{m}^{J}=\Gamma_{m}^{J} U$ is valid. Braid relations of intertwiners were found in Sect. 5. Since $i_{K}^{*}$ identified representations $\left(\pi_{K}, \mathscr{H}_{K}\right)$ and $\left(\pi_{0} \circ \varrho_{K}, \mathscr{H}_{0}\right)$, Eq. (5.32) can also be written in the form

$$
T\left(s_{2}\right) T\left(s_{1}\right) \varrho^{\left(s_{1}\right)}\left(\varepsilon_{J}\right)=\sum_{s_{2}^{\prime} s_{1}^{\prime}} T\left(s_{2}^{\prime}\right) T\left(s_{1}^{\prime}\right) R_{s_{2} s_{1}}^{s_{2}^{\prime} s_{1}^{\prime}}
$$

where $\varrho^{\left(s_{1}\right)}=\varrho_{L}$ if $s_{1}=\left(\begin{array}{c}\cdot \\ \\ L\end{array}\right)$. From Eq. (6.42) we obtain

$$
\begin{aligned}
\Gamma_{k}^{J} \Gamma_{l}^{J} \varepsilon_{J}= & \sum_{P, Q, S} \sum_{p, q, s} C\left[\begin{array}{lll}
P & J & S \\
p & k & s
\end{array}\right] C\left[\begin{array}{lll}
S & J & Q \\
s & l & q
\end{array}\right] \\
& \times i_{P p}^{*} \pi_{0}\left(T\left(\begin{array}{cc}
J \\
P & S
\end{array}\right) T\left(\begin{array}{cc}
J \\
S & Q
\end{array}\right) \varrho_{Q}\left(\varepsilon_{J}\right)\right) i_{Q q},
\end{aligned}
$$

while

$$
\begin{aligned}
\sum_{m n} \Gamma_{m}^{J} \Gamma_{n}^{J} \mathscr{R}_{k l}^{m n}= & \sum_{m n} \sum_{P, Q, S^{\prime}} \sum_{p, q, s^{\prime}} C\left[\begin{array}{ccc}
P & J & S^{\prime} \\
p & m & s^{\prime}
\end{array}\right] C\left[\begin{array}{ccc}
S^{\prime} & J & Q \\
s^{\prime} & n & q
\end{array}\right] \\
& \times \mathscr{R}_{k l}^{m n} i_{P p}^{*} \pi_{0}\left(T\left(\begin{array}{cc}
J \\
P & S^{\prime}
\end{array}\right) T\left(\begin{array}{cc}
J \\
S^{\prime} & Q
\end{array}\right)\right) i_{Q q} .
\end{aligned}
$$

Equation (6.47) and Eqs. (5.33), (5.34) for $R_{s_{2} s_{1}}^{s_{2} s_{1}^{\prime}}$ give

$$
\begin{aligned}
& T\left(\begin{array}{cc}
J \\
P & S
\end{array}\right) T\left(\begin{array}{cc}
J \\
S & Q
\end{array}\right) \varrho_{Q}\left(\varepsilon_{J}\right)=\sum_{S^{\prime}} T\left(\begin{array}{cc}
J \\
P & S^{\prime}
\end{array}\right) T\left(\begin{array}{cc}
J \\
S^{\prime} & Q
\end{array}\right) R_{(P S)(S Q)}^{\left(P S^{\prime}\right)\left(S^{\prime} Q\right)} \\
& R_{(P S)(S Q)}^{\left(P S^{\prime}\right)\left(S^{\prime} Q\right)}=(-1)^{S+S^{\prime}-P-Q} q^{\left(c_{P}+c_{Q}-2 c_{J}\right) / 2} e^{i \frac{\pi}{2}}\left\{\begin{array}{lll}
J & P & S \\
J & Q & S^{\prime}
\end{array}\right\}_{q} .
\end{aligned}
$$




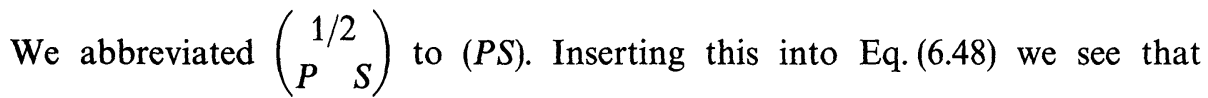
expressions (6.48) and (6.49) are equal if

$$
\begin{gathered}
\sum_{S, s} C\left[\begin{array}{lll}
P & J & S \\
p & k & s
\end{array}\right] C\left[\begin{array}{lll}
S & J & Q \\
S & l & q
\end{array}\right] R_{(P S)(S Q)}^{\left(P S^{\prime}\right)\left(S^{\prime} Q\right)} \\
=\sum_{s^{\prime}, m, n} C\left[\begin{array}{lll}
P & J & S^{\prime} \\
p & m & s^{\prime}
\end{array}\right] C\left[\begin{array}{lll}
S^{\prime} & J & Q \\
s^{\prime} & n & q
\end{array}\right] \mathscr{R}_{k l}^{m n} .
\end{gathered}
$$

This is called a "vertex-SOS transforamtion" in the work of Fröhlich et al. [41]. Summation over $S$ runs over physical representations $S=0, \frac{1}{2}, 1$ only. The braid matrices $R_{s_{1} s_{2}}^{s_{2}^{\prime} s_{1}^{\prime}}$ on the left-hand side are given by Eq. (6.50), while $\mathscr{R}_{k l}^{m n}$ and proportionality factors $c_{K J L}$ in (6.43) remain to be determined.

Inserting expressions (6.50) for $R_{\ldots}^{\cdots}$, and setting the coefficients $c_{K J L}=1$ would give

$$
\begin{aligned}
\sum_{S, s} & {\left[\begin{array}{lll}
P & J & S \\
p & k & s
\end{array}\right]\left[\begin{array}{lll}
S & J & Q \\
s & l & q
\end{array}\right](-1)^{S+S^{\prime}-P-Q} q^{\left(c_{P}+c_{Q}-2 c_{J}\right) / 2}\left\{\begin{array}{lll}
J & P & S \\
J & Q & S^{\prime}
\end{array}\right\}_{q} e^{i \frac{\pi}{2}} } \\
& =\sum_{s^{\prime}, m, n}\left[\begin{array}{lll}
P & J & S^{\prime} \\
p & m & s^{\prime}
\end{array}\right]\left[\begin{array}{lll}
S^{\prime} & J & Q \\
S^{\prime} & n & q
\end{array}\right] \mathscr{R}_{k l}^{m n} .
\end{aligned}
$$

Interpreting $\mathscr{R}_{k l}^{m n}$ as a canonical quantum group $\mathscr{R}$-matrix, Eq. (6.52) agrees with the defining equation for $6 j$-symbols, for generic $q$, except for the restriction in the sum over $S$. The equation is also valid whenever the tensor product $[J] \otimes[Q]$ of quantum group representations contains no unphysical subrepresentations [31]. If operators (6.48) and (6.49) are applied to $|\xi\rangle \in \mathscr{H}_{\boldsymbol{K} r}$, only the term with $Q=K, q=r$ contribute. It follows that Eq. (6.38) holds on a subspace of $\mathscr{H}_{\text {phys }}$ which contains $\mathscr{H}_{0}$ and $\mathscr{H}_{1 / 2}, m= \pm \frac{1}{2}$ if $J=\frac{1}{2}$, as claimed in Theorem 2, with $\mathscr{R}$ equal to the quantum group $\mathscr{R}$-matrix multiplied with $e^{-i \pi / 2}$.

If $J=1$, then $\varepsilon_{J}=-1$ and relation (6.38) is trivially satisfied by

$$
\mathscr{R}_{k l}^{m n}(J=1)=-\delta_{k}^{m} \delta_{l}^{n} .
$$

The corresponding local braid relations are

$$
\phi_{k}^{1} \phi_{l}^{1 \prime}=-\phi_{k}^{1 \prime} \phi_{l}^{1}
$$

This completes the proof of Theorem 2.

Let us finally explain the difficulty with the vertex-SOS transformation which prevented us from establishing local braid relations on the whole Hilbert space.

Consider again $J=\frac{1}{2}$ and suppose that matrix $\mathscr{R}_{m n}^{k l}$ is not diagonal [i.e. $\mathscr{R}_{m n}^{k l} \neq 0$ does not imply $(k l)=(m n)]$. The 3rd component of quantum isospin is conserved, i.e. $\mathscr{R}_{m n}^{k l}=0$ unless $k+l=m+n$. Therefore it follows that

$$
\mathscr{R}^{-1 / 2}-1 / 2 \neq 0 \text {. }
$$

The quantum group $\mathscr{R}$-matrix is nondiagonal. Consider the special case $P=Q=1$, $p=q=1$. Then the left-hand side of Eq. (6.52) is zero because $S=0, \frac{1}{2}, 1$ implies $s \neq \frac{3}{2}$. But the sum on the right-hand side consists of one nonvanishing term for $S^{\prime}=\frac{1}{2}$. Therefore, validity of Eq. (6.52) for all $P, Q$ is inconsistent with a nondiagonal $\mathscr{R}$-matrix. This argument does not depend on the exact form of $\mathscr{R}_{s_{1} s_{2}}^{s_{1}^{\prime} s_{2}^{\prime}}$, and the 
conclusion remains also valid when we admit arbitrary proportionality factors $c_{K J L}$, provided $c_{1 / 2} \quad 1 / 21^{\circ} c_{1} 1_{1 / 2} \quad 1 / 2 \neq 0$. If $c_{1 / 2} 1 / 21 \cdot c_{1 / 2} \quad 1 / 2$ were 0 , the field $\phi^{1 / 2}$ could either not make transitions to, or from, $\mathscr{H}_{1}$.

A similar problem was also encountered by Buchholz, Mack, and Todorov in a different approach [17].

6.4. Operator Product Expansions. It is straightforward to extend the considerations of Sect. 4.4 to obtain operator product expansions on the vacuum sector. If $\phi_{k}^{J, a}=\Gamma_{k}^{J} U_{a}$ with $U_{a} \in \mathscr{A}$ then

$$
\phi_{k}^{J_{2}, 2} \phi_{l}^{J_{1}, 1}=\sum_{J, m} c_{J_{2} J_{1}}\left[\begin{array}{ccc}
J & J_{2} & J_{1} \\
m & k & l
\end{array}\right] \phi_{m}^{J} \quad \text { on } \mathscr{H}_{0}
$$

with

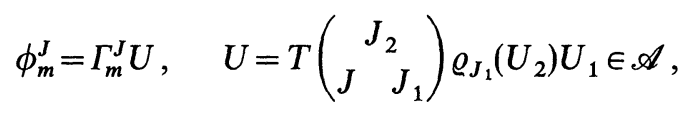

and structure constants $c_{J J_{2} J_{1}}$ as in Sect. 4.4.

Again this expansion does not remain valid on all sectors, but it is good enough to convert vacuum expectation values of products of field operators into vacuum expectation values of observables.

\section{A. Appendix. Local Braid Relations}

Suppose that the fields

$$
\phi_{k}^{J, i}=\Gamma_{k}^{J} U_{i}, \quad U_{i} \in \mathscr{A}, \text { unitary }
$$

are localized in disjoint intervals $I_{i}, i=1,2$ with $\zeta \notin I_{i}$. This means that endomorphisms $\varrho_{i}=\sigma_{U_{i}} \circ \varrho_{J}$ are localized in the intervals $I_{i}$. The point $\zeta$ entered the definition of $\varepsilon_{J}$. We show that braid relations

$$
\phi_{k}^{J, 1} \phi_{l}^{J, 2}=\sum_{m, n} \phi_{m}^{J, s} \phi_{n}^{J, 1} \mathscr{R}_{k l}^{m n}
$$

hold. Using Eq. (1.54), Definition (1.31) and homotopy invariance of $\varepsilon_{J}=\varepsilon_{\varrho_{J}}$, and Eq. (1.41) in turn we calculate

$$
\begin{aligned}
\phi_{k}^{J, 1} \phi_{l}^{J, 2} & =\Gamma_{k}^{J} \Gamma_{l}^{J} \varrho_{J}\left(U_{1}\right) U_{2} \\
& =\Gamma_{k}^{J} \Gamma_{l}^{J} \varepsilon_{J} \varrho_{J}\left(U_{2}\right) U_{1} \\
& =\sum_{m, n} \Gamma_{m}^{J} \Gamma_{n}^{J} \varrho_{J}\left(U_{2}\right) U_{1} \mathscr{R}_{k l}^{m n}=\sum_{m, n} \Gamma_{m}^{J} U_{2} \Gamma_{n}^{J} U_{1} \mathscr{R}_{k l}^{m n} \\
& =\sum_{m, n} \phi_{m}^{J, 2} \phi_{n}^{J, 1} \mathscr{R}_{k l}^{m n},
\end{aligned}
$$

as claimed in Eq. (A.2): To justify the use of homotopy invariance, $\varrho_{J}$ should be regarded as limit of localized endomorphisms as explained after Eq. (1.31), and $U_{i}$ must be limits of elements of local algebras $\mathscr{A}(I)$ with $\zeta \notin I$ as implied by the hypothesis of Remark 3. This restriction on $U_{i}$ eliminates the possibility of multiplying the fields with arbitrary elements of the center of $\mathscr{A}$. 


\section{B. Appendix. Table 1}

$$
\begin{aligned}
& C_{\frac{1}{2} \frac{1}{2}}\left[\begin{array}{cc}
\frac{1}{2} & \frac{1}{2} \\
0 & 0
\end{array}\right]_{q=-i}=e^{-i \frac{5 \pi}{8}}, \quad C_{\frac{1}{2} \frac{1}{2}}\left[\begin{array}{cc}
\frac{1}{2} & \frac{1}{2} \\
0 & 0
\end{array}\right]_{\mathrm{phys}}=e^{-i \frac{\pi}{8}}
\end{aligned}
$$

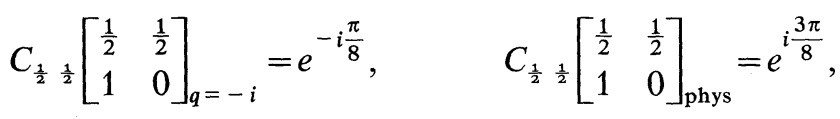

$$
\begin{aligned}
& C_{\frac{1}{2} \frac{1}{2}}\left[\begin{array}{cc}
\frac{1}{2} & \frac{1}{2} \\
0 & 1
\end{array}\right]_{q=-i}=e^{-i \frac{\pi}{8}}, \quad C_{\frac{1}{2} \frac{1}{2}}\left[\begin{array}{cc}
\frac{1}{2} & \frac{1}{2} \\
0 & 1
\end{array}\right]_{\text {phys }}=e^{i \frac{3 \pi}{8}}, \\
& C_{\frac{1}{2} \frac{1}{2}}\left[\begin{array}{cc}
\frac{1}{2} & \frac{1}{2} \\
1 & 1
\end{array}\right]_{q=-i}=e^{-i \frac{5 \pi}{8}}, \quad C_{\frac{1}{2} \frac{1}{2}}\left[\begin{array}{cc}
\frac{1}{2} & \frac{1}{2} \\
1 & 1
\end{array}\right]_{\mathrm{phys}}=e^{-i \frac{\pi}{8}} \\
& C_{0} 0\left[\begin{array}{cc}
\frac{1}{2} & \frac{1}{2} \\
\frac{1}{2} & \frac{1}{2}
\end{array}\right]_{q=-i}=\frac{1}{\sqrt{2}} e^{i \frac{3 \pi}{8}}, \quad C_{0} 0\left[\begin{array}{cc}
\frac{1}{2} & \frac{1}{2} \\
\frac{1}{2} & \frac{1}{2}
\end{array}\right]_{\mathrm{phys}}=-\frac{1}{\sqrt{2}} e^{-i \frac{\pi}{8}}, \\
& C_{1} 0\left[\begin{array}{cc}
\frac{1}{2} & \frac{1}{2} \\
\frac{1}{2} & \frac{1}{2}
\end{array}\right]_{q=-i}=\frac{1}{\sqrt{2}} e^{-i \frac{\pi}{8}}, \quad C_{1} 0\left[\begin{array}{cc}
\frac{1}{2} & \frac{1}{2} \\
\frac{1}{2} & \frac{1}{2}
\end{array}\right]_{\text {phys }}=\frac{1}{\sqrt{2}} e^{i \frac{3 \pi}{8}}, \\
& C_{0} 1\left[\begin{array}{ll}
\frac{1}{2} & \frac{1}{2} \\
\frac{1}{2} & \frac{1}{2}
\end{array}\right]_{q=-i}=\frac{1}{\sqrt{2}} e^{-i \frac{\pi}{8}}, \quad C_{0} 1\left[\begin{array}{cc}
\frac{1}{2} & \frac{1}{2} \\
\frac{1}{2} & \frac{1}{2}
\end{array}\right]_{\mathrm{phys}}=\frac{1}{\sqrt{2}} e^{i \frac{3 \pi}{8}} \\
& C_{1} 1\left[\begin{array}{cc}
\frac{1}{2} & \frac{1}{2} \\
\frac{1}{2} & \frac{1}{2}
\end{array}\right]_{q=-i}=\frac{1}{\sqrt{2}} e^{i \frac{3 \pi}{8}}, \quad C_{1} 1\left[\begin{array}{cc}
\frac{1}{2} & \frac{1}{2} \\
\frac{1}{2} & \frac{1}{2}
\end{array}\right]_{\mathrm{phys}}=-\frac{1}{\sqrt{2}} e^{-i \frac{\pi}{8}} \text {. }
\end{aligned}
$$

Acknowledgements. We are grateful to Professor I. M. Gelfand for encouragement, and to D. Buchholz, I. Frenkel, J. Fröhlich, N. Reshetikhin, J. Roberts, I. Todorov, and B. Tsygan for discussions. One of us (G.M.) would like to thank Arthur Jaffe for the warm hospitality extended to him at the Harvard Mathematics Department. This investigation was started while G.M. visited IHES Bures-sur-Yvette.

This work was supported in part by DOE grant DE-FG02-88ER2506500000200

\section{References}

1. Wick, G.G., Wigner, E.P., Wightman, A.S.: Intrinsic parity of elementary particles, Phys. Rev. 88, 101 (1952)

2. Haag, R., Kastler, D.: An algebraic approach to field theory. M. Math. Phys. 5, 848 (1964)

3. Doplicher, S., Haag, R., Roberts, J.E.: Local observables and particle statistics I, II. Commun. Math. Phys. 23, 199 (1971) and 35, 49 (1974)

4. Takesaki, H., Winnik, W.: Local normality in quantum statistical mechanics. Commun. Math. Phys. 30, 129 (1973)

5. Buchholz, D., Mack, G., Todorov, I.T.: The current algebra on the circle as a germ of local field theories. Nucl. Phys. B (Proc. Suppl.) 5B, 20 (1988)

6. Pressley, A., Segal, G.: Loop groups. Oxford: Oxford Science Publications 1986

7. Rehren, K.H., Schroer, B.: Einstein causality and artin braids. Nucl. Phys. B 312, 715 (1989) Rehren, K.H.: Locality of conformal fields in two dimensions. Exchange algebras on the light cone. Commun. Math. Phys. 116, 675 (1988)

8. Fredenhagen, K., Rehren, K.H., Schroer, B.: Superselection sectors with braid group statistics and exchange algebras, I: General theory. Commun. Math. Phys. 125, 201-226 (1989) 
9. Fredenhagen, K., Rehren, K.H., Schroer, B.: As reported in B. Schroer: New kinematics (statistics and symmetry) in low-dimensional QFT with applications to conformal QFT ${ }_{2}$, to be published in Proc. VXII ${ }^{\text {th }}$ Int. Conf. on Differential Geometric Methods in Theoretical Physics, 1989

10. Frenkel, I., Lepowsky, J., Meurman, A.: Vertex operators and the monster. New York: Academic Press 1988

11. Fröhlich, J., Gabbiani, F., Marchetti, P.A.: Superselection structure and statistics in threedimensional local quantum theory, preprint ETH-TH/89-22

Fröhlich, J., Gabbiani, F., Marchetti, P.A.: Braid statistics in three-dimensional local quantum theory, ETH-TH/89-36, to be publ. in Proceedings "Physics, Geometry, and Topology," Banff 1989

12. Fredenhagen, K.: Structure of superselection sectors in low-dimensional quantum field theory, to be publ. in: XVII International conference on Differential Geometric Methods in Theoretical Physics: Physics and Geometry, Davis 1989

13. Doplicher, S., Roberts, J.E.: Why there is a field algebra with a compact gauge group describing the superselection structure in particle physics, June 1989

Doplicher, S., Roberts, J.E.: $C^{*}$-algebras and duality for compact groups: Why there is a compact group of internal gauge symmetries in particle physics. In: VIII ${ }^{\text {th }}$ International Congress on Mathematical Physics, Marseille 1986, Mebkhout, M., Sénéor, R. (eds.)

Doplicher, S., Roberts, J.E.: Endomorphisms of $C^{*}$-algebras, cross products and duality for compact groups. Ann. Math. (to appear)

14. Drinfel'd, V.G.: Quantum groups. Proc. ICM 798 (1987)

15. Fröhlich, J.: Statistics of fields, the Yang-Baxter equation and the theory of knots and links. In: Nonperturbative quantum field theory. t'Hooft, G. et al. (eds.). New York: Plenum Press 1988

16. Moore, G., Reshetikhin, N.: A comment on quantum symmetry in conformal field theory. Nucl. Phys. B 328, 557 (1989)

17. Buchholz, D., Mack, G., Todorov, I.T.: As reported in I. Todorov. In: Proceedings of Conf. on Quantum Groups, Clausthal Zellerfeld, July 1989

18. Alvarez-Gaumé, L., Gomez, C., Sierra, G.: Hidden quantum symmetry in rational conformal field theories. Nucl. Phys. B 310 (1989)

Alvarez-Gaumé, L., Gomez, C., Sierra, G.: Quantum group interpretation of some conformal field theories. Phys. Lett. 220B, 142 (1989)

Alvarez-Gaumé, L., Gomez, C., Sierra, G.: Duality and quantum groups, CERN-TH-5369/89

19. Felder, G., Fröhlich, J., Keller, G.: On the structure of unitary conformal field theory II: Representation theoretic approach ETH-TH/89-12

20. Tsuchiya, A., Kanie, Y.: Vertex operators in the conformal field theory of $\mathbf{P}^{1}$ and monodromy representations of the braid group. Lett. Math. Phys. 13, 303 (1987)

21. Temperley, H., Lieb, E.: Relation between the percolation and the colouring problem. Proc. Roy. Soc. (London) 251 (1971)

22. Jones, V.: Index for subfactors. Invent. Math. 72, 1 (1982) and in: Braid group, knot theory and statistical mechanics. Yang, C.N., Ge, M.L. (eds.). Singapore: World Scientific 1989

23. Hamermesh, N.: Group theory, Reading, MA: Addison Wesley 1962, Chap. 3-17

24. Lüscher, M., Mack, G.: Global conformal invariance in quantum field theory. Commun. Math. Phys. 41, 203 (1975)

25. Mack, G.: Introduction to conformal invariant quantum field theories in two dimensions. In: Nonperturbative quantum field theory. t'Hooft, G. et al. (eds.). New York: Plenum Press 1988

26. Schomerus, V.: Diplomarbeit, Hamburg, 1989

27. Buchholz, D., Schulz-Mirbach, H.: Work in preparation

28. Gelfand, I.M., Shilov, G.E.: Generalized functions vol. I. New York: Academic Press 1960

29. Connes, A., Evans, D.E.: Embedding of U(1)-current algebras of classical statistical mechanics. Commun. Math. Phys. 121, 507 (1988)

30. Longo, R.: Index of subfactors and statistics of quantum fields I, II. Commun. Math. Phys. 126, 217 (1989) and 130, 285-309 (1990)

31. Kirillov, A.N., Reshetikhin, N.: Representations of the algebra $U_{q}(s l(2))$, q-orthogonal polynomials and invariants of links, preprint LOMI E-9-88, Leningrad 1988

32. Segal, I.: Causally ordered manifolds and groups. Bull. Am. Math. Soc. 77, 958 (1971)

33. Lusztig, G.: Modular representations of quantum groups. Contemp. Math. 82, 59 (1989) 
34. Ganchev, A., Petkova, V.: $U_{q}(s l(2))$ invariant operators and minimal theories fusion matrices. Trieste preprint, IC/89/158 (June 89)

35. Pasquier, V.: Etiology of IRF models, Commun. Math. Phys. 118, 365 (1988)

36. Moore, G., Seiberg, N.: Polynomial equations for rational conformal field theories. Phys. Lett. 212 B, 451 (1988)

37. Dotsenko, V.S., Fateev, V.A.: Four point correlation functions and the operator algebra in the 2-dimensional conformal quantum field theories with the central charge $c<1$. Nucl. Phys. B 251 [FS 13], 691 (1985)

Dotsenko, V.S., Fateev, V.A.: Conformal algebra and multipoint correlation functions in 2-dimensional statistical models. Nucl. Phys. B 240 [FS 12], 312 (1984)

38. Pasquier, V., Saleur, H.: Common structures between finite systems and conformal field theories through quantum groups, submitted to Nucl. Phys. B [FS]

39. Wenzl, H.: Hecke algebras of type $A_{n}$ and subfactors. Inv. Math. 92, 349 (1988)

Wenzl, H.: Quantum groups and subfactors of type B, C, E. UC San Diego preprint, July 1989

40. Bouwknegt, P., McCarthy, J., Pilch, K.: Free field realization of WZNW models, BRST complex and its quantum group structure, submitted to Phys. Lett. B (1989)

41. Fröhlich, J., King, C.: Two-dimensional conformal field theory and 3-dimensional topology, Commun. Math. Phys. (to appear)

Felder, J., Fröhlich, J., Keller, G.: Braid matrices and structure constants for minimal conformal models. Commun. Math. Phys. (to appear)

42. Mack, G.: Duality in quantum field theory. Nucl. Phys. B 118, 445 (1977), esp. p. 456

Communicated by A. Jaffe 